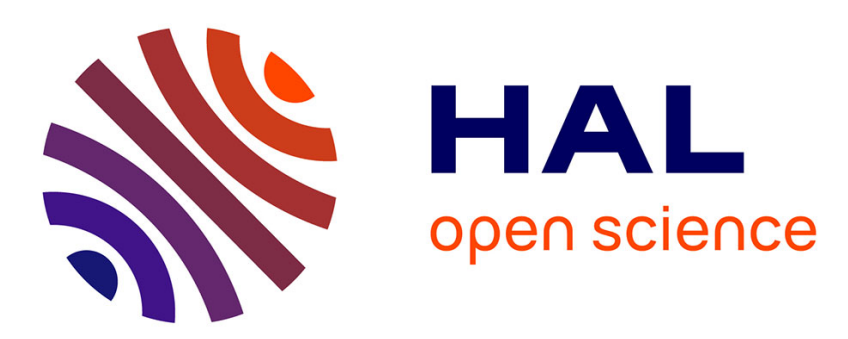

\title{
Fundamentals and exchange rate forecastability with simple machine learning methods
}

\author{
Christophe Amat, Tomasz Michalski, Gilles Stoltz
}

\section{To cite this version:}

Christophe Amat, Tomasz Michalski, Gilles Stoltz. Fundamentals and exchange rate forecastability with simple machine learning methods. 2018. halshs-01003914v6

\section{HAL Id: halshs-01003914 \\ https://shs.hal.science/halshs-01003914v6}

Preprint submitted on 28 May 2018

HAL is a multi-disciplinary open access archive for the deposit and dissemination of scientific research documents, whether they are published or not. The documents may come from teaching and research institutions in France or abroad, or from public or private research centers.
L'archive ouverte pluridisciplinaire HAL, est destinée au dépôt et à la diffusion de documents scientifiques de niveau recherche, publiés ou non, émanant des établissements d'enseignement et de recherche français ou étrangers, des laboratoires publics ou privés. 


\title{
Fundamentals and exchange rate forecastability with simple machine learning methods ${ }^{\text {th }}$
}

\author{
Christophe Amat ${ }^{\mathrm{a}}$, Tomasz Michalski ${ }^{\mathrm{b}, *}$, Gilles Stoltz ${ }^{\mathrm{c}}$ \\ ${ }^{a}$ Ecole Polytechnique, Palaiseau, France \\ ${ }^{b}$ HEC Paris - GREGHEC, Jouy-en-Josas, France \\ ${ }^{c}$ HEC Paris - CNRS, Jouy-en-Josas, France
}

\begin{abstract}
Using methods from machine learning we show that fundamentals from simple exchange rate models (PPP or UIRP) or Taylor-rule based models lead to improved exchange rate forecasts for major currencies over the floating period era 1973-2014 at a 1-month forecast horizon which beat the no-change forecast. Fundamentals thus contain useful information and exchange rates are forecastable even for short horizons. Such conclusions cannot be obtained when using rolling or recursive OLS regressions as used in the literature. The methods we use - sequential ridge regression and the exponentially weighted average strategy, both with discount factors - do not estimate an underlying model but combine the fundamentals to directly output forecasts.
\end{abstract}

JEL Classification: C53, F31, F37

Keywords: exchange rates, forecasting, machine learning, purchasing power parity, uncovered interest rate parity, Taylor-rule exchange rate models

\section{Introduction}

We show that fundamentals from "classic" exchange rate models and Taylor-rule exchange rate models are useful in forecasting short-term changes in exchange rates for major currencies. Using prediction techniques borrowed from machine learning that come with performance guarantees - sequential ridge regression with discount factors and the exponentially weighted average strategy with discount factors - we are broadly able to improve forecasting forward the 1-month exchange rate for the floating rate period 1973-2014 in terms of the usual root mean square error (RMSE) criterion, and

\footnotetext{
We would like to thank an anonymous referee, Philippe Bacchetta, Charles Engel, Refet Gürkaynak, Robert Kollmann, Jose Lopez, Evren Örs, Cavit Pakel, Martin Puhl, Romain Rancière, Helene Rey, Barbara Rossi, Michał Rubaszek, Kenneth D. West and the seminar participants at Bilkent University, HEC Paris, the National Bank of Poland, OECD, UN, EEA 2015 conference, Royal Economic Society 2015, 8th Financial Risks International Forum 2015 (Institut Louis Bachelier) for helpful comments and discussions. All remaining errors are ours.

* Corresponding author

Email addresses: christophe.amat@polytechnique.edu (Christophe Amat), michalski@hec.fr (Tomasz Michalski), stoltz@hec.fr (Gilles Stoltz)

Preprint submitted to Elsevier 
obtain lower Theil ratios than the no-change (random walk) prediction. Fundamentals from purchasing power parity models (PPP), uncovered interest rate parity (UIRP), and Taylor-rule exchange rate models - which traditional exchange rate forecasting literature (based on OLS methods) has failed to find effective (see Rossi, 2013 for a recent comprehensive review) - in fact carry valuable information for out-of-sample forecasting even at short-term horizons ${ }^{1}$. We obtain similar success with predicting the direction of change of exchange rates and several economic criteria, and also conduct various robustness checks.

These results have important implications both for economic theory and policy. For example, Alvarez et al. (2007) argue that if exchange rates are random walks, monetary policy concentrated on short-term interest rates does not have typically assumed nominal or real effects, but rather affects only risk premia. More generally, it has been frustrating for researchers in international economics that forces stipulated by the simplest and most intuitive models about one of the most important prices - exchange rates - did not seem to be empirically detectable.

Our findings are contrary to the consensus in the literature led by the famous Meese and Rogoff (1983) result on the inability of short-term forecasts based on fundamentals to outperform the hard-to-beat no-change/random walk predictions ${ }^{2}$. The improvements in forecasts using fundamentals presented here (up to $1.3 \%$ in terms of RMSE) are not large - the fundamentals we consider do not help to add a lot of predictive content relative to the one of "no change" from the previous observed exchange rate - but are substantial (and often statistically significant at conventionally accepted levels) with respect to the existing literature. The observations of Engel and West (2005) may well be valid: current and past fundamentals may have low correlations with future exchange rate values. Fundamentals may have little predictive power against an exchange rate that can be approximated by a random walk, though they may add some useful information.

Why should we use these methods and not others? A common feature of the methods we use is the following: they attempt to avoid a problem known as overfitting past data, which means being able to reconstruct well - using the forecasting equations - previous data (having a good "in-sample" prediction error) but with poor future predictions (delivering a poor "out-of-sample" prediction error). Such problems have permeated the exchange-rate prediction literature for the last 35 years, from Meese and Rogoff (1983) to Fratzscher et al. (2015).

These methods do not estimate the coefficients of some underlying model, but rather, treat the coefficients as numbers to be chosen (not estimated) over time in order to form good predictions. Indeed, they do not rely on stochastic modeling of exchange rates (e.g., in terms of a linear combination of the fundamentals plus stochastic errors). They have appealing features (described in Section 3.2) as theoretical guarantees (bounds) on their performance can be proven. In particular, their RMSEs converge, as the number

\footnotetext{
${ }^{1}$ Forecastability of exchange rates over longer time periods - such as 2-year forward forecasts and beyond - has been established in the literature, see Section 6. Forecastability is not the same thing as predictability, which has also been studied; see Footnote 25.

${ }^{2}$ The rare exceptions are that of Clark and West (2006) that demonstrate the predictability of a UIRPbased model or Wright (2008) that uses Bayesian model averaging methods. See a longer discussion in Section 6.
} 
of instances to be predicted increases, to a quantity that is (always) not larger than the RMSE of the no-change/random walk prediction, and even not larger than the RMSE of the best fixed linear forecasting equation (for certain methods). The latter value is in general strictly smaller than the RMSE of the no-change/random walk prediction, so asymptotically such methods should beat the random walk if fundamentals indeed drive the exchange rate. It can also be shown that no such guarantee can be achieved for conventional (rolling or recursive) OLS regressions. Moreover, we apply our methods to fundamentals precisely identified by classic economic models of exchange rates, and do not run "kitchen-sink" regressions where many different and not necessarily related fundamentals are mixed together. The following are crucial differences between our work and that of other studies like Li et al. (2015) and Plakandaras et al. (2015), which forecast exchange rates with different machine learning techniques: the methods we use come with relevant performance guarantees, solve outright a statistical problem encountered by many previous studies, and use only one (very limited) set of theory-motivated fundamentals at a time.

We stress that both the discount factors and (hyper)parameters of our methods (a learning rate for the exponentially weighted average strategy with discount factors, a regularization factor for sequential ridge regression with discount factors) are not imposed from above or set arbitrarily. In particular, our methods do not rely on educated guesses about important parameters, as for example in Wright, 2008 for Bayesian model averaging methods and in other papers, where typically the performance of methods at hand are reported for several, hand-picked, parameter values). Note finally that the methods we implement are general forecasting methods that were not specifically designed for exchange rate forecasting, but proved to work well in other problems (e.g., air quality and electricity consumption forecasting; see Cesa-Bianchi and Lugosi, 2006, Mauricette et al., 2009, and Stoltz, 2010).

Machine learning methods. The first method - the exponentially weighted average strategy with discount factors - allows us to weigh fundamentals by their past performance. Coefficients chosen over time are convex and are proportional to the exponential of the average past performance of each fundamental. These vary over time as the performance of each fundamental evolves. If certain fundamentals always perform poorly, they are rapidly (almost) discarded, though by construction all coefficients are non-zero even if some may be close to zero. The success of this algorithm, which identifies the best predictors in any period, may be due to the channel exhibited in the scapegoat exchange rates model of Bacchetta and van Wincoop (2004) (tested for in Fratzscher et al., 2015): traders consider some of the "most fashionable" fundamentals (in some periods) that eventually drive the exchange rates. When we look closer at the weights we see that quite often there is one prevailing fundamental that carries most of the information for forecasting exchange rates; details are provided in Appendix D, Figures D.1-D.3 of the online supplementary material (Amat et al., 2018b).

The second method - sequential ridge regression with discount factors ${ }^{3}$ - resembles OLS regression, but prevents the in-sample overfitting issue encountered by adding a

\footnotetext{
${ }^{3}$ Ridge regression was introduced by Hoerl and Kennard (1970) in a stochastic and non-sequential setting. However, it turns out that the exact same method can be analyzed in a sequential non-stochastic setting, leading to guarantees of another nature, namely in the form (7). We will discuss these machinelearning analyses in Section 3.2.
} 
regularization term to an otherwise standard OLS regression. The regularization term prevents the smallest possible in-sample error being obtained, which typically leads to better generalization (smaller out-of-sample error). The elastic net method considered by Li et al. (2015) proceeds in a similar fashion, with a combination of Euclidean and absolute-value regularization. However, it comes with no clear theoretical performance guarantees like those which can be shown for ridge regression.

For both methods, we consider variants of the basic machine-learning formulations which include discount factors. Discounting puts more weight on recent forecasting errors. This allows us to accommodate structural breaks that may be present in the data (e.g., changes in the conduct of monetary policy, crisis times, etc.) better than the original formulations. This is also true for any changes in the behavior of market participants. It should be noted that our discounts are of a polynomial and not geometric form (as used in most of the economic literature). This is because it can be proven that theoretical guarantees can be associated with the former (see Section 3.2) but not the latter in our context (see Theorems 2.7 and 2.8 of Cesa-Bianchi and Lugosi, 2006 for a discussion of the performance of different discounting methods).

The data used and the scope of our study. In our study, aside from the specific machinelearning methods, we use data and forecast evaluation methods that are standard in the literature, as discussed in Rossi (2013). We use lagged fundamentals and do not detrend, filter, or seasonally adjust the data in any way. We use single-equation methods and our benchmark for comparison is the no change in the exchange rate prediction. We study the floating currency pairs for major industrialized countries, (i) for the entire period since the breakup of the Bretton Woods fixed exchange rate system (1973-2014), and (ii) for the period 1999-2017 for a subset of currencies using "real-time" data. We use end-of-month exchange rates (with one reading of the exchange rate at the end of the month).

We conduct several robustness checks, such as trying out different (sub)samples, variations on the exact form of the fundamentals included, etc., and our results remain valid. Furthermore, an investigation of statistical properties of the errors shows that our improvement is uniform over time, rather than driven by a few well-predicted instances. There are several themes discussed by Rossi (2013) that are also relevant to our study. Traditional linear estimation methods (rolling or recursive OLS regressions) with our fundamentals fail to forecast exchange rates better than the no change in exchange rates prediction. With the addition of several fundamentals (aggregating information) we do not get gains in prediction precision - that is, parsimonious forecasting equations work best. Fundamentals from the same time period as the forecast (true observed values) perform poorly.

Our study is not comprehensive nor exhaustive in nature. Our goal is not to provide the best possible forecasts of exchange rates by finding fundamentals from the large set considered in the vast literature that have the strongest predictive power (or aggregating the information contained therein for example in hundreds of different time series). We are interested in showing that we can detect fundamentals driving exchange rates even at short horizons, and argue for the usefulness of machine learning methods in applications to well-known and hard-to-crack economic problems such as predicting inflation or output. 


\subsection{Organization of the paper}

In Section 2, we present the fundamentals that we shall consider. Then, in Section 3, we discuss the analysis methods and put them in perspective with respect to standard linear regressions. Next, in Section 4 we present the data, while in Section 5 we discuss the results. Section 6 contains a literature review and Section 7 concludes. Supplementary online material (Amat et al., 2018b) contains further details regarding the methods and results tables.

\section{Fundamentals considered}

We study "classic" fundamentals stemming from the simple exchange rate models of the 1970s and Taylor-rule based models. As described in Rossi (2013), there are four main reasons for this: (i) these fundamentals come from exchange rate models that involve basic relationships in standard international economics, (ii) they have been extensively used in the literature, (iii) studies have shown that at short-time horizons, forecasting methods based on them were not successful with respect to the no-change forecast ${ }^{4}$ benchmark, and (iv) data is widely available for long periods for a large set of countries and does not require any transformations (as for example, productivity data does).

\subsection{General framework}

In the following, "home" or "domestic" refers to the U.S., while "foreign" refers to some other country whose exchange rate w.r.t. the U.S. dollar is studied. by

The general forecasting equation for exchange rate change for a currency pair is given

$$
\widehat{s}_{t+1}-s_{t}=\alpha_{t}+\sum_{j=1}^{N} \beta_{j, t} f_{j, t},
$$

where $s_{t}$ is the logarithm of the exchange rate (home currency units per unit of the foreign currency) at time $t$, the intercept $\alpha_{t}$ and slope coefficients $\beta_{j, t}$ are to be chosen based on information available up to time $t$, and the $f_{j, t}$ are the $N$ fundamentals considered at time $t$. Throughout the paper we will impose ${ }^{5}$ that $\alpha_{t}=0$. The time unit is months.

By the exchange rate, we here mean the end-of-the month value.

The coefficients $\beta_{j, t}$ (and $\alpha_{t}$ when intercepts are considered, which is not the case here) are usually picked through rolling or recursive OLS regression. Rossi (2013) states that "the literature has been focusing mainly on rolling or recursive window forecasting schemes (see West, 2006), where parameters are reestimated over time using a window of recent data", and Cheung et al. (2005) mention that "the convention in the empirical exchange rate modeling literature of implementing rolling regressions [was] established

\footnotetext{
${ }^{4}$ We remind readers that no other set of fundamentals has been as yet shown to beat such forecasts in a consistent manner either. See Footnote 25 on the predictability of exchange rates with Taylor-rule model-based fundamentals.

${ }^{5}$ Affine forecasting equations can be handled via only one of our two forecasting methods, namely sequential ridge regression with discount factors, and do not offer an improvement upon the results shown in the paper. Details are available upon request. The other method - the exponentially weighted average strategy with discount factors - is not applicable to affine forecasting equations.
} 
by Meese and Rogoff (1983)." This is because these gave the best performance and no other method consistently beat them. In this paper, we revisit this convention and consider other methods for picking these coefficients. We do not write "estimate these coefficients", as this would imply some underlying model with true parameters $\alpha$ and $\beta$. We will be interested solely in forecasting performance, and not at all in the existence or use of a model ${ }^{6}$.

Before recalling how rolling and recursive OLS regressions proceed to that end, and presenting alternative methods stemming from the machine learning field, we discuss the fundamentals of interest.

\subsection{Fundamentals from PPP, UIRP, monetary, and Taylor-rule models}

We are only interested in the predictive performance of the forecasting methods we use. We allow forecasting equations with both different (which is going to be our base scenario) and equal (we shall call them "coupled") coefficients for home and foreign fundamentals relating to the same measured quantity. There are good reasons to allow for different coefficients. Identical series, even if pertaining to a similar economic concept, may be either unavailable or measured completely differently in any two countries (given that they are provided by independent institutions using a range of methods). Hence, the best weights picked may differ because the elasticities of response of investors to purportedly similar fundamentals in each country may and indeed should differ ${ }^{7}$. A further issue that is pertinent while analyzing data series over such long periods is that central banks sometimes change their ways of conducting monetary policy - for example by following inflation targets or Taylor rules - at different times across countries which as is well known - may also change the way in which exchange rates react to fundamentals.

The first time-series of fundamentals is formed by inflation differentials, which are also used for instance in the relative purchasing power parity model. The associated forecasting equation is given by

$$
\widehat{s}_{t+1}-s_{t}=\beta_{1, t} \pi_{t}-\beta_{2, t} \pi_{t}^{\star},
$$

where $\pi_{t}$ and $\pi_{t}^{\star}$ are respectively the home and foreign measures of 12-month inflation rates available (known) at time $t$. In what follows, a " $\star$ " will denote the variables for the

\footnotetext{
${ }^{6}$ We avoid using the word "model" here, as we will not have to assume that some true underlying model of the differences exists like

$$
s_{t+1}-s_{t}=\alpha_{t}+\sum_{j=1}^{N} \beta_{j} f_{j, t}+\varepsilon_{t+1},
$$

from which, by estimation of the unknown coefficients $\beta_{j}$ some prediction $\widehat{s}_{t+1}$ could be obtained

${ }^{7}$ The most flagrant example is perhaps money stocks: for the United Kingdom and Sweden only M0 aggregates, and for Italy and the Netherlands only M2, are available for the entire period considered, and not M1 as for other countries. M0, M1 and M2 money aggregates are typically correlated, but they obviously measure different things, and the relationship between those within the same country may not be stable. Even for countries for which M1 measures are available, these contain different components depending on the country. Such issues unfortunately exist to some extent for every fundamental considered, and is a general problem in the literature. Due to the asymptotic properties of the methods, we wanted to obtain the longest series possible for the largest number of currencies and had to - given the data available - sacrifice standardization to some extent.
} 
foreign country ${ }^{8}$.

The forecasting equations using the fundamentals from the uncovered interest rate parity model are of the form

$$
\widehat{s}_{t+1}-s_{t}=\beta_{1, t} i_{t \rightarrow t+1}-\beta_{2, t} i_{t \rightarrow t+1}^{\star},
$$

where $i_{t \rightarrow t+1}$ and $i_{t \rightarrow t+1}^{\star}$ are the short run (money-market) interest rates at home and in the foreign country respectively. These would be the interest rates at which investors could place money at time $t$ for the period $t$ to $t+1$ and be observed by them in real time.

The third time-series of fundamentals consists of changes in money stocks and outputs. The simplest monetary model (flexible price, Frenkel-Bilson model) states that exchanges rates can be modeled as linear combinations of the form

$$
s_{t}=\alpha+\phi\left(m_{t}-m_{t}^{\star}\right)-\omega\left(y_{t}-y_{t}^{\star}\right),
$$

where $m_{t}$ and $m_{t}^{\star}$ are the logarithms of the money stocks at time $t$, and $y_{t}$ and $y_{t}^{\star}$ the logarithms of outputs. Here, $\alpha, \phi$ and $\omega$ denote some true underlying parameters for the model. As indicated several times, we will not rely on the existence of such a monetary model, and only consider it to extract the fundamentals it proposes and substitute them into our forecasting equations. Indeed, after lagging the said fundamentals by a month (to account for known and present but not forward values), differencing the above equation ${ }^{9}$, and allowing for decoupled fundamentals, we consider ${ }^{10}$ the forecasting equations

$$
\widehat{s}_{t+1}-s_{t}=\beta_{1, t} \Delta m_{t}-\beta_{2, t} \Delta m_{t}^{\star}-\beta_{3, t} \Delta y_{t}+\beta_{4, t} \Delta y_{t}^{\star},
$$

where $\Delta x_{t}=x_{t}-x_{t-1}$ denotes the change between periods $t-1$ and $t$ for a variable $x$. In (4), the parameters $\beta_{1, t}, \ldots, \beta_{4, t}$ are to be picked over time as in (2) and (3).

We also investigate whether all of the fundamentals mentioned above taken together are able to predict changes in the exchange rate ${ }^{11}$.

Lastly, we also consider whether the fundamentals extracted from a Taylor-rule based exchange-rate model (which have been found successful in establishing the predictability

\footnotetext{
${ }^{8}$ The relative PPP forecasting equation would actually stipulate $\widehat{s}_{t+1}-s_{t}=\beta_{1, t} \Delta p_{t+1}-\beta_{2, t} \Delta p_{t+1}^{\star}$, where $\Delta p_{t+1}$ is the change in the price level between periods $t$ and $t+1$. However, as the change in the future price level is unknown at time $t$, we use past price changes (inflation) in our forecasting exercise.

${ }^{9}$ We proceeded this way as some of the methods we use - in particular the exponentially weighted average strategy with discount factors - require direct predictions of the exchange rate change. An alternative is to find some "equilibrium" exchange rate driven by the aforementioned fundamentals and use the deviation of the current exchange rate from that "theoretical" exchange rate as the fundamental. Doing this does not qualitatively change our results.

${ }^{10}$ Recall that the model does not need to hold - it merely helps us decide which fundamentals to consider in our forecasting equations.

${ }^{11}$ The form in which the fundamentals are included does not appear to matter, at least for sequential ridge regression with discount factors. We produced forecasts using fundamentals from the PPP and monetary models for our main sample also using price or output indices in levels or the monetary stock: our qualitative conclusions do not change for sequential ridge regression with discount factors. We also investigated fundamentals extracted from the other popular monetary flexible-price model that also involves differences in interest rates, which in our forecasting equations would add terms $i_{t}-i_{t-1}$ and $i_{t}^{\star}-i_{t-1}^{\star}$ as predictors. We do not show the results as they were very close to the performance of the forecasting equation (4). We included these fundamentals, however, when considering forecasts with al fundamentals at hand to see whether aggregating information from many different time-series helps.
} 
of exchange rate changes, as discussed in Section 6) are also useful in forecasting. We use the fundamentals from the most parsimonious version of equation (7) from Molodtsova and Papell (2009) for our forecasting equation:

$$
\widehat{s}_{t+1}-s_{t}=\beta_{1, t} \pi_{t}^{\star}-\beta_{2, t} \pi_{t}+\beta_{3, t} \widetilde{y}_{t}^{\star}-\beta_{4, t} \widetilde{y}_{t}+\beta_{5, t} i_{t-1 \rightarrow t}^{\star}-\beta_{6, t} i_{t-1 \rightarrow t},
$$

where $\widetilde{y}_{t}$ and $\widetilde{y}_{t}^{\star}$ are present measures of the output gaps. Therefore, our Taylor-rule fundamentals are the inflation, output gaps, and lagged interest rates.

The actual data time-series used are further discussed in Section 4.

Coupled fundamentals. In forecasting equations (2)-(5), fundamentals of home and foreign countries pertaining to the same concept can also have the same weights. For example, the forecasting equation inspired by the PPP model in (2) is then $\widehat{s}_{t+1}-s_{t}=$ $\beta_{1, t}\left(\pi_{t}-\pi_{t}^{\star}\right)$. We will refer to this situation as coupled fundamentals, and perform robustness checks using this forecasting equation. Note that it still falls under the general umbrella of (1), only with $N / 2$ coefficients to pick instead of $N$, and with the $f_{j, t}$ now referring to the differences between home and foreign fundamentals.

\section{Methodology}

As indicated above, forecasts $\widehat{s}_{t+1}$ of 1-month ahead exchange rates $s_{t+1}$ are based on the forecasting equations (2)-(5). A forecasting method consists of a rule for picking the coefficients $\beta_{j, t}$ over time based on past and present information. Several such methods are presented in Sections 3.1 and 3.2, namely conventional rolling and recursive OLS regressions (in brief - Section 3.1), as well as two other methods stemming from the field of sequential learning (in detail - Section 3.2).

As discussed in detail in Appendix B of the online supplementary material (Amat et al., 2018b), we evaluate the forecasting ability of these methods through their (out-ofsample) root mean square error, which is computed by working with a training period of $t_{0}=120$ months, as is standard in the literature. (This training period is not taken into account in the evaluation of performance.) To determine whether the improvements in RMSE of one method over another are statistically significant, we use the Diebold and Mariano (1995) test, referred to as the DM test in the following; we in fact do so on bootstrapped data. For the sake of completeness (and though not fully applicable in our setting, see Appendix B.3 of the online supplementary material) we also report the results of the tests by West $(1996)$ and Clark and West $(2006,2007)$ for determining significant improvements over the no-change prediction only (denoted $\mathrm{CW}$ hereafter). Last, we compute the Theil ratio of the RMSE predicted by the forecasting method of interest over that of the no-change prediction. A ratio below 1 means that the given method gave a lower RMSE than the no-change prediction.

\subsection{Forecasting methods (part 1): classical methods}

We now present the various forecasting methods considered in this paper. We will do so in some generality, encompassing all of the forecasting equations (2)-(5) under the umbrella of (1):

$$
\widehat{s}_{t+1}-s_{t}=\sum_{j=1}^{N} \beta_{j, t} f_{j, t} .
$$


We recall that in our view, the coefficients $\beta_{j, t}$ are to be picked according to some rule; they do not need to be understood as estimating some unknown underlying true value.

The no-change forecasting method. The first strategy consists of choosing $\beta_{j, t}=0$ for all $j$ at each round $t$, that is, of forecasting $\widehat{s}_{t+1}$ with $s_{t}$. We call this the no-change forecasting method.

Rolling OLS regression. This is the most standard technique in the literature, "the convention" as Cheung et al. (2005) state. The idea is to truncate the available information to account for the most recent relationships between variables that can change over time because of policy changes (for example, a change to Taylor-rule based monetary policy), structural changes in the economy (such as shifting relationships between the money stock and inflation), etc. For this forecasting method, we need to choose the length $h$ of the estimation window, which we also use for the training period, as is standard in the literature (see Molodtsova and Papell, 2009): $h=t_{0}=120$ months. The rolling OLS regression picks, for months $t \geqslant h$,

$$
\left(\beta_{1, t}, \ldots, \beta_{N, t}\right)=\underset{\beta_{1}, \ldots, \beta_{N} \in \mathbb{R}}{\arg \min } \sum_{\tau=t-h+1}^{t}\left(s_{\tau}-s_{\tau-1}-\sum_{j=1}^{N} \beta_{j} f_{j, \tau-1}\right)^{2} .
$$

Recursive OLS regression. This is another standard technique in the literature. It consists of choosing, for each month $t$,

$$
\left(\beta_{1, t}, \ldots, \beta_{N, t}\right)=\underset{\beta_{1}, \ldots, \beta_{N} \in \mathbb{R}}{\arg \min } \sum_{\tau=1}^{t}\left(s_{\tau}-s_{\tau-1}-\sum_{j=1}^{N} \beta_{j} f_{j, \tau-1}\right)^{2},
$$

i.e., all past time instances, and not only the $h$ most recent ones, are used to form the prediction.

\subsection{Forecasting methods (part 2): simple machine learning algorithms}

The forecasting methods considered in this study stem from the field of machine learning, where they are already standard methods for the robust online prediction of quantitative phenomena by aggregation of basic predictors (fundamentals). The book by Cesa-Bianchi and Lugosi (2006) summarizes research performed on and around them over the period 1989-2006.

These methods have been applied in the following fields, among others: forecasting air quality (see, e.g., Mauricette et al., 2009; Mallet, 2010; Debry and Mallet, 2014) and electricity consumption (see, e.g., Devaine et al., 2013; Gaillard and Goude, 2015). We use them below "by the book", i.e., we provide no tweaking and apply them "as are" in the references above. We underline that therefore these methods are not ad hoc ones constructed solely for the problem of predicting exchanges rates.

The theoretical out-of-sample guarantees that they come with are of the following form: for all possible bounded sequences of exchange rates and fundamentals, their 
predictions are such that

$\sum_{t=1}^{T}\left(\widehat{s}_{t}-s_{t-1}-\sum_{j=1}^{N} \beta_{j, t-1} f_{j, t-1}\right)^{2}-\inf _{\beta^{\dagger} \in \mathcal{F}} \sum_{t=1}^{T}\left(\widehat{s}_{t}-s_{t-1}-\sum_{j=1}^{N} \beta_{j}^{\dagger} f_{j, t-1}\right)^{2} \leqslant B(\mathcal{F}, T)$,

with $B(\mathcal{F}, T) \ll T$ and where $\mathcal{F} \subset \mathbb{R}^{N}$ is some comparison class (the forecasting methods presented below are independent of the choice of $\mathcal{F}$, only the bound $B(\mathcal{F}, T)$ is sensitive to it). We explain below what we mean by a comparison class, i.e., a set of candidates for the underlying expression of the exchange rates in terms of linear combinations of fundamentals. With the algorithms presented below, such linear combinations can be given by $\mathcal{F}$ equal to the set of all point mass combinations (weights that equal 1 for one fundamental and are null for all others), or equal to some bounded Euclidean ball of $\mathbb{R}^{N}$ (e.g., all linear weights with Euclidean norm bounded by some constant $U$, whereby $U$ appears in the bound).

In particular, from (6), dealing separately with the training period of size $t_{0}$ (for which an error bounded by something of the order of $t_{0}$ is made in the worst case), dividing by $T-t_{0}$ and taking square roots, we get the following guarantee on out-of-sample RMSEs:

$$
\begin{array}{r}
\limsup _{T \rightarrow \infty}\left\{\sqrt{\frac{1}{T-t_{0}}} \sum_{t=t_{0}+1}^{T}\left(\widehat{s}_{t}-s_{t-1}-\sum_{j=1}^{N} \beta_{j, t-1} f_{j, t-1}\right)^{2}\right. \\
\left.-\inf _{\beta^{\dagger} \in \mathcal{F}} \sqrt{\frac{1}{T-t_{0}} \sum_{t=t_{0}+1}^{T}\left(\widehat{s}_{t}-s_{t-1}-\sum_{j=1}^{N} \beta_{j}^{\dagger} f_{j, t-1}\right)^{2}}\right\} \leqslant 0 .
\end{array}
$$

Let us comment on these out-of-sample guarantees. First, they rely on no stochastic modeling; they are achieved for all possible bounded sequences of exchange rates and fundamentals, and are thus deterministic. In fact, the bound $B(\mathcal{F}, T)$ also depends on the range in which the exchange rates and the fundamentals lie, but is a uniform bound. Second, what these out-of-sample guarantees truly ensure is that the forecasting method has asymptotically an average performance as good as or better than that of the best constant linear combination in $\mathcal{F} \subset \mathbb{R}^{N}$, i.e., the best fixed model with coefficients in $\mathcal{F}$. However, of course, as mentioned above, such a model does not need to exist, since no stochastic modeling assumption is required. It just turns out that the methods presented below mimic the performance of the best model if applicable (or of the best fixed linear forecasting equation otherwise). Put differently, we can interpret the infimum in (6) (the error suffered by the best pick in the comparison class) as measuring some approximation error (how well in hindsight the fundamentals can predict the exchange rates), while the $B(\mathcal{F}, T)$ term is a sequential estimation error (the price to pay for facing a sequential rather than batch problem).

One needs to note that getting close to or slightly better than the performance of the best fixed linear forecast is not necessarily good enough for obtaining great forecasts; the best fixed linear forecast can have poor performance. This may be true in the exchange rates setting where it was hard until now to obtain forecastability using fundamentals 
(see Rossi, 2013). In other fields of application mentioned above - such as forecasting of electricity consumption or air quality - such forecasts do well. Lastly, we note that it can be shown that out-of-sample guarantees like (7) cannot be achieved by rolling or recursive OLS regression (details available upon request). Performance guarantees on the latter (if any exist) thus would require at minimum stochastic modeling of the sequence of exchange rates.

Is it better to use coupled or decoupled fundamentals? The short answer is: there is no theoretical reason to prefer one over the other. Indeed, while the approximation errors of the decoupled version are always smaller than those of coupled versions (simply because they correspond to an infimum taken on a larger set), the bounds $B(\mathcal{F}, T)$ on the sequential estimator errors in (6) always increase with the number $N$ of independent fundamentals, and are thus larger with decoupled than with coupled fundamentals. As the total errors suffered by our forecasting methods are the sums of these two errors, their empirical behavior as $N$ increases is unclear, since theoretically they can either increase or decrease.

EWA: the exponentially weighted average strategy with discount factors. This was introduced in the early 90s by Vovk (1990) and Littlestone and Warmuth (1994), and further studied and developed by - among others - Cesa-Bianchi et al. (1997); Cesa-Bianchi (1999); Auer et al. (2002). Here, we present a minor generalization - already considered, e.g., by Mauricette et al. (2009) - in which past prediction instances get slightly more weight when they are more recent. This forecasting method involves different parameters: a sequence $\left(\eta_{t}\right)$ of positive numbers referred to as learning rates, a non-negative number $\gamma$ called the discount factor, and a positive number $\kappa>0$ called the discount power. It picks the weights according to

$$
\beta_{j, t}=\frac{1}{Z_{t}} \exp \left(-\eta_{t} \sum_{\tau=1}^{t}\left(1+\frac{\gamma}{(t+1-\tau)^{\kappa}}\right)\left(s_{\tau}-s_{\tau-1}-f_{j, \tau-1}\right)^{2}\right),
$$

where $Z_{t}$ is a normalization factor: ${ }^{12}$

$$
\begin{aligned}
Z_{t}=2 \exp \left(-\eta_{t}\right. & \left.\sum_{\tau=1}^{t}\left(1+\frac{\gamma}{(t+1-\tau)^{\kappa}}\right)\left(s_{\tau}-s_{\tau-1}\right)^{2}\right) \\
& +\sum_{j=1}^{N} \exp \left(-\eta_{t} \sum_{\tau=1}^{t}\left(1+\frac{\gamma}{(t+1-\tau)^{\kappa}}\right)\left(s_{\tau}-s_{\tau-1}-f_{j, \tau-1}\right)^{2}\right)
\end{aligned}
$$

Because of this factor and the exponent function in the equations defining the $\beta_{j, t}$, this strategy is referred to as the exponentially weighted average strategy (the EWA

\footnotetext{
${ }^{12}$ This is the version for decoupled fundamentals. With coupled fundamentals, the number of summands is reduced by a factor of 2 in the defining sum over $j$ and concomitantly the factor 2 in the first term in the definition of $Z_{t}$ is replaced by 1 . This is because this first term accounts for the no-change prediction, which in its decoupled version as in (9) corresponds to the difference of 2 zero variations (one for the value of the currency of each country), instead of 1 zero variation (of the exchange rate) in the coupled case.
} 
strategy in short). Note that the weights obtained form a sub-convex weight vector: the components $\beta_{j, t}$ are non-negative and sum up to something smaller than or equal to 1. (The missing mass with respect to 1 can be interpreted as a measure of the confidence that no change will take place between $s_{t}$ and $s_{t+1}$.)

A study of the theoretical out-of-sample guarantees of EWA in the presence of discount factors was undertaken in Stoltz (2010, Theorem 3), see also Cesa-Bianchi and Lugosi $(2006, \S 2.11)$, and can be interpreted as follows in our context. For all choices of $\gamma, \kappa$, and non-increasing sequences $\left(\eta_{t}\right)$ such that, as $t \rightarrow+\infty$,

$$
t \eta_{t} \longrightarrow+\infty \quad \text { and } \quad \eta_{t} \sum_{\tau=1}^{t} \frac{1}{\tau^{\kappa}} \longrightarrow 0,
$$

the desired guarantee $(7)$ holds for the set $\mathcal{F}=\mathcal{M}$ of all point-mass vectors. For instance, $\eta_{t}=1 / \sqrt{t}$ and $\kappa=2$ would be suitable choices but many other choices associated with the desired theoretical guarantees exist.

More precisely ${ }^{13}$ the bound $B(\mathcal{M}, T)$ of (6) equals

$$
B(\mathcal{M}, T)=\frac{2 \ln N}{\eta_{T}}+\sum_{t=1}^{T} \frac{\eta_{t}}{2} L^{2}+L \sum_{t=1}^{T}\left(\exp \left(2 L \eta_{t} B_{t-1}\right)-1\right)
$$

where

$$
B_{t-1}=\sum_{\tau=1}^{t-1} \frac{\gamma}{\tau^{\kappa}}
$$

and $L$ is a bound on the quadratic errors $\left(s_{t}-s_{t-1}\right)^{2}$ and $\left(s_{t}-s_{t-1}-f_{j, t-1}\right)^{2}$ as $t$ and $j$ vary.

SRidge: sequential ridge regression with discount factors. The issue with (recursive or rolling) OLS regressions is that they tend to overfit past data, i.e., they lead to good in-sample predictions but poor out-of-sample ones. To prevent this, one can add what is called a regularization term to the squared error to help control (reduce) the range of components $\beta_{j, t}$ of the linear vector picked. The smallest in-sample error is typically achieved with large-valued coefficients, which are exactly the ones that the included regularization term tries to avoid. Focusing solely on the in-sample error tends to be detrimental when generalizing results, i.e., when making out-of-sample predictions. We implement below this regularization strategy for a forecasting method called sequential ridge regression with discount factors.

Ridge regression was introduced by Hoerl and Kennard (1970) in a stochastic (nonsequential) setting. What follows relies on recent new analyses of ridge regression in the machine learning community - see the papers by Vovk (2001) and Azoury and Warmuth (2001), as well as the survey in the book by Cesa-Bianchi and Lugosi (2006). Sequential

\footnotetext{
${ }^{13}$ See Chapter 6 of the technical report by Mallet et al. (2007), which is cited as the main ingredient in the proof of Theorem 3 of Stoltz (2010). Also, readers aware of the bounds that can be proven for the square loss in our context may note that we do not exploit its exp-concavity with the help of a well-chosen fixed learning rate; this is for practical performance purposes.
} 
ridge regression (without discount factors and for a constant regularization factor $\lambda \geqslant 0$ ) picks the weights

$$
\left(\beta_{1, t}, \ldots, \beta_{N, t}\right)=\underset{\beta_{1}, \ldots, \beta_{N} \in \mathbb{R}}{\arg \min _{j=1}}\left\{\lambda \sum_{j=1}^{N} \beta_{j}^{2}+\sum_{\tau=1}^{t}\left(s_{\tau}-s_{\tau-1}-\sum_{j=1}^{N} \beta_{j} f_{j, \tau-1}\right)^{2}\right\} .
$$

We now state the associated performance bound as in (6). This comes in terms of the classes $\mathcal{F}_{U}$ of linear weights with Euclidean norm bounded by $U>0$ (where the bound holds for all $U>0$ simultaneously). We denote by $L$ a bound on the exchange rates and the fundamentals, whose value is such that $s_{t} \in[-L, L]$ and $f_{j, t} \in[-L, L]$. Then, for all $U>0$, the bound $B\left(\mathcal{F}_{U}, T\right)$ of $(6)$ equals $^{14}$

$$
B\left(\mathcal{F}_{U}, T\right)=\lambda U^{2}+4 N L^{2}\left(1+\frac{N T L^{2}}{\lambda}\right) \ln \left(1+\frac{T L^{2}}{N \lambda}\right) .
$$

In particular, when $\lambda$ is well chosen (e.g., of the order of $\sqrt{T}$ ), one has

$$
B\left(\mathcal{F}_{U}, T\right)=\mathcal{O}(\sqrt{T} \ln T) \ll T .
$$

Recursive OLS regression corresponds to the special case when $\lambda=0$, but no theoretical bound is offered in this case.

As announced above, we have used a common variant of the classical ridge regression presented above so as to focus more on recent observations. This is obtained via discounting: sequential ridge regression (with discount factor $\gamma$ and for a constant regularization factor $\lambda \geqslant 0)$ picks the weights

$$
\begin{aligned}
& \left(\beta_{1, t}, \ldots, \beta_{N, t}\right) \\
& =\underset{\beta_{1}, \ldots, \beta_{N} \in \mathbb{R}}{\arg \min }\left\{\lambda \sum_{j=1}^{N} \beta_{j}^{2}+\sum_{\tau=1}^{t}\left(1+\frac{\gamma}{(t+1-\tau)^{\kappa}}\right)\left(s_{\tau}-s_{\tau-1}-\sum_{j=1}^{N} \beta_{j} f_{j, \tau-1}\right)^{2}\right\} .
\end{aligned}
$$

EWA and SRidge in practice: how to choose their (hyper)parameters. Following machine learning terminology, we call hyperparameters the parameters (learning rate, regularization factor, discount factor, etc.) of the learning method at hand. In our simulation

${ }^{14}$ The original bound of Azoury and Warmuth (2001, Theorem 4.6), as cited by Cesa-Bianchi and Lugosi (2006, Section 11.7) in the special case $\lambda=1 / 2$, reads

$$
\lambda \sum_{j=1}^{N} \beta_{j}^{2}+N \ln \left(1+\frac{T L^{2}}{N \lambda}\right) \max _{t \leqslant T}\left(\widehat{s}_{t}-s_{t}\right)^{2} .
$$

Nevertheless, this does not imply a logarithmic bound as the term $\max _{t \leqslant T}\left(\widehat{s}_{t}-s_{t}\right)^{2}$ may be large, as pointed out by Gerchinovitz (2011, page 66). One can show (details upon request) that this maximum is however never larger than

hence our stated bound.

$$
4 \max \left\{L^{2}, \frac{N T L^{4}}{\lambda}\right\}
$$


study we will not report the performance of the EWA and SRidge methods for several well-chosen sets of hyperparameters, as is usual in the literature (as for example in Wright, 2008 for Bayesian model averaging methods, or in other papers, where typically the performance of the methods at hand are reported for several, hand-picked values of the hyperparameters). We instead use a sequential grid search (i.e., perform a grid search at each step, which is a natural approach already considered in Devaine et al., 2013) and thus only report the results obtained for a single instance of the method. This instance does not require any previous knowledge since the hyperparameters are set online and do not have to be determined in advance. Details are provided in Appendix C.1 of the online supplementary material (Amat et al., 2018b).

\section{Data and fundamentals used}

Our main sample includes 12 floating exchange rates for major industrial economies from March 1973 to December 2014 (we have at most 502 data points per currency). We considered the same currencies as Molodtsova and Papell (2009). Machine learning methods should have superior performance, especially over long time periods, given the guarantees on their performance discussed in Section 3 - hence our desire to use the longest time-series possible. We use end-of-month exchange rates which are taken from the IMF's IFS database. We tried to extend the same data series for fundamentals as used in Molodtsova and Papell (2009), but some of them were discontinued. As a result, we tried to find the closest substitutes possible for the entire period 1973-2014 from similar sources (IMF, OECD) through Datastream. For robustness checks we also used real-time data obtained from the OECD for the period February 1999 to April 2017. A detailed description of the data is given in Appendix A of the online supplementary material (Amat et al., 2018b).

We principally study the behavior of 12 major floating currencies that are active throughout the 1973-2014 period. The introduction of the Euro in 1999 constrains the sample for some continental Europe currencies (FRF/USD, DEM/USD, ITL/USD, NLG/USD, PTE/USD). Even for the active currencies (USD/GBP, JPY/USD, CHF/USD, $\mathrm{CAD} / \mathrm{USD}$, SEK/USD, USD/AUD, DNK/USD), however, it was not possible to obtain all fundamentals time-series for the entire 1973-2014 period (see details for each in Appendix A of the online supplementary material).

Our fundamentals are formed as follows. The inflation differentials are calculated as 12-month changes in consumer price indexes (CPI). We use a money market rate or 3month interest rate differentials for the interest rate-based fundamentals. For differences in money stock growth and output growth we use the preceding 12-month trends in these variables. We do not detrend, filter or seasonally adjust the data. The output gaps for the Taylor-rule fundamentals are percentage deviations from "potential" output that were computed including (i) a linear trend, (ii) a quadratic trend, (iii) a linear and quadratic trend, and (iv) a Hodrick-Prescott filter using the data available prior to the date for which the output gap was calculated.

The data set thus created is freely available for download (Amat et al., 2018a). 


\section{Results}

\subsection{Results for "classic" fundamentals}

Our baseline results are shown in Tables 1 and 2, and include the RMSE $\times 100$ of the no-change prediction ${ }^{15}$ (column 1) and the Theil ratios (columns 2, 6, 10 and 14) of predictions, as well as the corresponding $p$-values of the CW and DM tests. The former are referred to as "CW $p$-values" and are found in columns 3, 7, 11 and 15 . As explained in Appendix B.3 of the online supplementary material (Amat et al., 2018b), these should be used with extreme caution in our context. Indeed, they correspond to fundamentals being useful $\left(H_{1}^{\prime}\right)$ or not $\left(H_{0}^{\prime}\right)$ in some existing underlying model - a matter of predictability not necessarily associated with better forecasting ability. On the other hand, the $p$-values for the DM tests are more reliable in our context, as the DM test is a general test for comparing forecasting abilities (see Appendix B.2 of the online supplementary material for details $\left.{ }^{16}\right)$. These $p$-values, are called "DM $p$-values" (columns 5, 9, 13 and 17) and evaluate the hypothesis $H_{0}$ that the difference in the forecasting performance of the method under scrutiny is not significantly better than that of the no-change prediction, against the alternative hypothesis $H_{1}$ that it is.

The sets of columns correspond to the forecasting methods: rolling OLS regression, recursive OLS regression, sequential ridge regression with discount factors and the exponentially weighted average strategy with discount factors respectively, while the sets of rows correspond to the sets of fundamentals discussed in Section 2.2: PPP fundamentals, UIRP fundamentals, monetary model fundamentals, and all of these combined.

Results allowing different coefficients on the same fundamentals across countries. Table 1 shows that we obtain better predictions than the no-change prediction for sequential ridge regression with discount factors and the exponentially weighted average strategy with discount factors methods using the PPP or UIRP fundamentals, allowing for different coefficients for fundamentals across countries. The improvements, however, are small (not higher than $1.3 \%$ in terms of RMSE) and most of the time we cannot reject the hypothesis that the forecasting methods being compared perform similarly. The exponentially weighted average strategy with discount factors is more successful as a method - both for the PPP and the UIRP it can improve upon the no-change prediction for 10 of the 12 currency pairs. In the PPP fundamentals case, this performance is statistically superior at the $10 \%$ level in 8 of the 12 cases according to the DM tests (and in 9 of the 12 according to the $\mathrm{CW}$ tests). Sequential ridge regression with discount factors is less successful, being able to outperform the no-change prediction in 7 of the 12 cases for PPP fundamentals, and 6 of the 12 for UIRP fundamentals. There is no evidence that by using monetary fundamentals or all fundamentals one could improve broadly upon the no-change prediction with these methods.

\footnotetext{
${ }^{15}$ These may differ for the same currency pair for different sets of fundamentals given that the latter are available for different periods (see the data Tables A.8-A.9 in Appendix A of the online supplementary material), so the forecasted periods may vary.

${ }^{16}$ In particular, for the DM tests, we compute $p$-values based on bootstrapping the time series at hand, as is commonly done in the literature, see references cited in Appendix B.2 of the online supplementary material.
} 
Results with coupled fundamentals. Table 2 presents the results in which we enforce symmetric coefficients across countries for the same fundamentals. The results are weaker than those where different coefficients were allowed. We are now able to beat random walk predictions (as signaled by Theil ratios $<1$ ) only for the exponentially weighted average strategy with discount factors for PPP ( 8 of the 12 currency pairs) and UIRP (10 of the 12), but very few of these are statistically significant at conventional levels.

Remarks on the performance of our methods. The conclusions we can make from Tables 1 and 2 are quite interesting. While allowing for different coefficients across countries, we can claim forecastability of exchange rates for PPP and UIRP fundamentals using the exponentially weighted average strategy with discount factors, which has not been previously demonstrated for such a wide range of currencies at such short range. The gains in RMSE compared to the random walk are small and often not statistically significant as judged by the DM test. However, comparing to existing literature - as surveyed by Rossi (2013) - these gains are substantial.

The theoretical tradeoff signalled in Section 3.2 between parsimony and adding fundamentals is not clear, which is visible in our results. For the decoupled case, when we combine all fundamentals - in the spirit of the information aggregation ability of machine learning techniques - we do not gain in terms of forecasting accuracy. Sometimes, the forecasts even turn out to be less precise in terms of RMSE. On the other hand, constraining the coefficients on fundamentals to be equal across countries leads to worse forecasting results for PPP- and UIRP-based fundamentals. However, when we use all fundamentals together (our version of "all but-the-kitchen-sink" regression) in the last panel of Table 2, we obtain better results for the coupled (constrained coefficients) case. Indeed, we can beat the no-change prediction in 8 of the 12 cases for the exponentially weighted average strategy with discount factors, while only in 5 of the 12 in the decoupled case (cf. the corresponding panel in Table 1).

There is a cost suffered by our methods when adding fundamentals that do not perform well. To rephrase this in the terms used in Section 3.2, the potential decrease in approximation error when considering all fundamentals is too small to be compensated by a larger sequential estimation error linked to dealing with more fundamentals. The important criterion is then the (a priori, of course, unknown) information content of the fundamentals used for forecasting that, themselves, by construction are predicted probably with errors based on their past values. Our results therefore show that parsimonious (PPP- and UIRP-based) forecasting equations seem to be preferable in our setting, as also seen in other studies of exchange rate predictability using different methods. This may be an indication that the different types of fundamentals we study contain essentially the same information ${ }^{17}$. However, the better performance of the settings with one fundamental allowing for differing coefficients across countries, rather than restricting them to be equal, shows that the concern that different monetary policy conducts or imperfectly comparable economic time-series pose additional difficulties in exchange rate

\footnotetext{
${ }^{17}$ Theoretically, with additional assumptions, there are links between the theories from which our "classic" fundamentals are taken. Interest rates could be high in a high inflation environment, and with the same risk premium both UIRP and PPP would predict a depreciation of such a currency. If inflation is primarily a monetary phenomenon, high monetary growth would be correlated with high inflation and interest rates.
} 
forecasting is warranted. One should underline that the performance of OLS methods worsens with the number of fundamentals, but no difference in forecasting performance between equations with constrained/unconstrained coefficients could be determined for these methods.

Performance of the rolling and recursive OLS strategies. The same fundamentals seem not to have much forecasting power when evaluated using the classical methods considered in the literature - either rolling or recursive OLS regression - no matter whether we allow for asymmetry in coefficients on the same fundamentals or not. For none of the currency pairs do we obtain a similar number of improvements in terms of RMSE over the no-change prediction ${ }^{18}$. These conclusions are in line with the existing empirical literature documenting that fundamentals do not allow for systematic improvements in forecasting (see Rossi, 2013).

Direct comparison of the machine learning and OLS strategies. Instead of making an indirect comparison between our forecasting methods and those typically used in the literature, we can directly compare their performance by comparing the Theil ratios (of the machine learning method's RMSE with respect to the OLS methods' ones) and perform an appropriate (bootstrapped) DM test. The results of this exercise are shown in Table 3, as well as in Table D.10 of the online supplementary material (Amat et al., 2018b). The first conclusion is that both methods do globally better than the OLS methods while comparing the Theil ratios, no matter what type of fundamentals are considered (even for monetary-model fundamentals and all fundamentals when the machine learning methods were not successful in beating the no-change prediction) or what assumption on the (a)symmetry of coefficients on fundamentals across countries is made. Interestingly, the largest gains are obtained when all fundamentals are used (last sub-table of each table) - up to $15.6 \%$ in terms of RMSE (for the SEK/USD pair in the coupled version of the exponentially weighted average strategy with discount factors vs. rolling OLS regression). This is partly because of the weak performance of the OLS methods as the number of fundamentals used grows, which has been signaled in the literature (Rossi, 2013), while

\footnotetext{
18 In Table 1 the $p$-value $4.1 \%$ of the DM test for USD/GBP prediction, using a rolling OLS regression based on PPP fundamentals, may seem odd given that the Theil ratio equals 1.0025 and the DM statistic takes the value -0.2934 . This is a consequence of the evaluation of the $p$-value through bootstrapping: it turns out that on the bootstrapped samples, the performance of the rolling OLS regressions are even worse (much higher Theil ratios, more negative DM statistics). The distribution of bootstrapped DM statistics is thus highly shifted to the left, which makes the value -0.2934 observed on the original data unlikely under the distribution of the bootstrapped DM statistic. (A detailed analysis of this can be provided upon request.) Such patterns occur in the analyzed data for OLS methods, as can be seen in other entries in the same table. This does not mean that a statistically significant gain in forecasting accuracy is achieved, as no gain in forecasting accuracy is achieved in the first place. The original Theil ratio is still $>1$ or, equivalently, the DM statistic is negative. This only illustrates the fact that the bootstrapping correction is (only) of help to better evaluate potential improvements in forecasting accuracy; it is meaningless in cases where no improvement exists in the first place. Similar observations are documented in the literature: see, e.g., Rogoff and Stavrakeva (2008, Table 2) and Mark and Sul (2001, Tables 6 and 7). The bootstrapping in the latter concerns Theil ratios (not DM statistics) but the issue is similar, of course; they always check whether, in discussing the results, the Theil ratios on the original data are $<1$. As for Rogoff and Stavrakeva (2008), they write (in their Appendix A): "A significant and positive DMW test implies that the structural model outperforms the random walk." Hence, positivity of the DM statistic is not to be forgotten.
} 
the effect of this increasing number is milder in the case of our machine learning methods (see, e.g., Mauricette et al., 2009).

Finally, we note that some descriptive statistics show that this on average good performance of our new methods does not come at the cost of local disasters. On the contrary, the forecasting errors seem to be uniformly better over time. Indeed, when computing the quantiles of the difference between the forecasting error of the no change minus the one of the methods under scrutiny (see Appendix B.1 of the online supplementary material), as shown in Table D.14 of Appendix D of the online supplementary material, we see that these differences are uniformly much larger for sequential ridge regression with discount factors and the exponentially weighted average strategy with discount factors than for the recursive or rolling OLS regressions when the methods exhibit forecastability for a given currency pair. By "uniformly" we mean here that quantiles of the same order for two sequences of differences are almost always ranked in the same manner, those corresponding to sequential ridge regression with discount factors and the exponentially weighted average strategy with discount factors (first sequence) being larger than those for rolling or recursive OLS regressions (second sequence).

\subsection{Robustness checks}

We conducted several robustness checks to verify whether our results were not due to some quirk related to the samples that we constructed. First, we decided to also consider 6 -month inflation, money stock or output changes in the decoupled version to see whether a different way of constructing fundamentals matters. This did not qualitatively change our results.

Next, we ran again the basic combinations of fundamentals and methods from Tables 1 and 2 on our data trimmed to shorter samples: 1980-2014, the post-Plaza accord period 1985-2014, the post ERM-crisis period 1992-2014, and 1973-2006, so as to see whether the inclusion of a particular period (e.g., the high inflation period of 1970s or the post financial crisis period) drives the results. We show the results for the 1980-2014 sample in Table D.15 of the online supplementary material. What we observe in general is that the forecasting properties of our methods still hold, though the observed gains against the no-change prediction are typically smaller, but - especially for the forecasts based on parsimonious sets of fundamentals (PPP, UIRP) - still often statistically significant. This may indicate that in practice, longer data series are in general beneficial for the (asymptotic) guarantees to kick in to minimize RMSE, as indicated in Section 3.2.

We also tried to work with what we call "absolute" fundamentals. This means that instead of inflation differentials we directly used price, money and output levels as substitutes for the PPP and monetary model fundamentals ${ }^{19}$. The results here are weaker. We can still obtain improvements with sequential ridge regression with discount factors. A problem arises, though, with the exponentially weighted average strategy with discount factors in that the forecast errors involving deviations of the exchange rates from the absolute fundamentals considered are much larger that those for relative fundamentals used in our original exercise. Also, the weights assigned to the absolute fundamentals

\footnotetext{
${ }^{19}$ The UIRP-model inspired forecasting equation has no such counterpart. "Absolute" fundamentals $A_{t}$ were created by adding price, money or output changes to an initial exchange rate in our data sets. Then the deviation of the actual nominal rate from its thus calculated "fundamental" value $A_{t}-s_{t}$ was used to predict $s_{t+1}-s_{t}$.
} 
by the method tend to be small (i.e., the predictions output by the method are close to the no-change ones). This is a documented fact: this method does not easily allow for fundamentals that err much and are consistently dominated by a fundamental with systematically good performance (a situation which arises in our case, the latter being the no-change prediction). This is in contrast with sequential ridge regression with discount factors, which can correct the fundamentals for proper scaling.

Last, we worked with the actual values of the fundamentals. That is, instead of "predicting" the next period's change in fundamentals using past values, we fed in the actual values that occurred in the course of economic activity. As stated in the literature starting with Meese and Rogoff (1983), these do not lead to good or better forecasts.

\subsection{Directional tests}

A different, though secondary, measure of forecast quality that has been employed in the literature is "directional tests", i.e., tests of whether forecasting methods are able to predict the direction of change of the exchange rate better than a fair coin toss. Although the methods considered in this paper are not constructed to be efficient in this regard - as their focus is on controlling RMSE (see Section 3.2) - we have tested how the predictions obtained by these methods fare in this case. In Table 4 , as well as in Table D.11 of the online supplementary material, we show the percentage of successes of each method (OLS regressions as well as sequential ridge regression with discount factors and the exponentially weighted average strategy with discount factors) in forecasting the direction of change of exchange rates with the corresponding (bootstrapped) DM $p$-value of the test of the difference with a fair coin toss.

The patterns that emerge are interesting. Machine learning methods are able to predict the correct direction in several cases $55 \%$ of the time (with a maximum of $60.8 \%$ ) - improvements which are often statistically significant. For machine-learning methods, the success in predicting RMSE goes typically hand in hand with the success of directional predictions for the decoupled fundamentals. This is not true for the forecasts made while constraining the coefficients to be equal, nor by OLS-based methods; in these cases, as the number of fundamentals included grows, they do better in "directional tests" while failing in terms of RMSE.

\subsection{Economic evaluation}

In Table 5 we show various economic criteria used to evaluate the economic benefits one would obtain using our forecasting methods as opposed to a random walk. This involves calculating investment returns, and we do this for the exponentially weighted average strategy with discount factors, generally obtaining improvements in terms of the RMSE, as shown in Table 1, which shows that detected improvements would also lead to superior performance from an investor's perspective.

The idea is to assess the utility of the forecasts from an investor's point of view, who builds one-period portfolios using information known at that given period under standard mean-variance preferences with a target standard deviation (see West et al., 1993, Della Corte et al., 2012 and Della Corte and Tsiakas, 2012). It is assumed in this context that investors make decisions on investing in money market rates of different countries, taking into consideration the forecasted movements of the exchange rates. Then various performance measures of such portfolios are compared to see whether some 
forecasting method generates predictions that can be profitably used by investors over those of a no-change model. (See Appendix $\mathrm{C}$ of the online supplementary material for further details.)

We consider the standard measures in the literature: a direct comparison of annualized returns for a portfolio using forecasts and a random walk, an annualized performance fee (in bps), annualized premium return (in bps), and annualized Sharpe and Sortino ratios. Given the oft-discussed concern that optimal portfolio selection applied to investment problems with exchange rates frequently leads to large portfolio weights (implying investors are taking very risky bets), we also study portfolio allocation when we restrict the weights $w_{1, t}, \ldots, w_{K, t}$ on the $K=7$ currencies $^{20}$ so that $w_{1, t}^{2}+\ldots+w_{K, t}^{2} \leqslant c$, which is a standard constraint in the machine-learning literature. (See Appendix $\mathrm{C}$ of the online supplementary material for the technical details of how this can be achieved. When we use such a constraint, we provide the corresponding value for $c$; otherwise, we write "no $c "$ in our tables.)

We see that in line with the results shown in Table 1 obtained from RMSE comparisons of forecasts, our methods would add value to investors using them over strategies based on no-change predictions for PPP or UIRP fundamentals. For the PPP, annualized returns are $13.48 \%$ for the EWA-based strategy as opposed to $11.83 \%$ for the random walk. However, investment risk is also an important factor in decision-making and investors would be willing to pay a performance fee of $229 \mathrm{bps}$, or would obtain a premium return of $277 \mathrm{bps}$, by sticking to our forecasts. Both the Sharpe and the Sortino ratios would be higher for the EWA-forecast based portfolios versus no-change ones (0.84 vs. 0.64 and 1.08 vs. 0.68 respectively). The same holds for UIRP-fundamentals. Returns, the Sharpe ratios, and the Sortino ratios, for a portfolio based on EWA-algorithm forecasts are higher $(12.13 \%$ vs. $11.44 \%, 0.78$ vs. 0.62 , and 1.38 vs. 0.65 respectively) and investors would be willing to pay a performance fee of 192 bps or obtain a premium return of 268 bps. This no longer holds true for predictions based on "monetary model" fundamentals or when we use all fundamentals together. It can be seen that constraining the weights (with $c=1$ or $c=2$ ) greatly improves the results in every relevant way for all types of fundamentals. We achieve a premium return larger than 360 bps with the UIRP fundamental and constraint $c=2$.

In Table D.16 of Appendix D of the online supplementary material we repeat the exercise, dropping the DNK/USD exchange rate, which is highly correlated with the SEK/USD and CHF/USD ones. This correlation creates some instability in the weights and leads to, in our calculations, optimally shorting one of these currencies with a very high weight and going long in the others. We see that the results of the economic criterion evaluation greatly improve when dropping the DNK/USD exchange rate, especially for "monetary model" fundamentals and when we use all fundamentals together. In Tables D.17 and D.18 of the online supplementary material we impose an additional constraint on the weights, requiring that their sum on the currencies is smaller than 1 , i.e., $w_{1, t}+\ldots+w_{K, t} \leqslant 1$.

We therefore observe that overall, investors would benefit from using our forecasts instead of the random walk prediction, and would be willing to pay up to slightly more than 360 bps for our best predictions in our basic scenarios.

\footnotetext{
${ }^{20}$ We consider: GBP, JPY, CHF, CAD, SEK, DNK and AUD, but not the currencies that later merged to become the Euro as they would mean a shorter overall time horizon.
} 


\subsection{Real-time data results}

We re-ran our methods using real-time data. The use of real-time data can help to alleviate the concern that forecasting performance is improperly evaluated because the information known at the time by market participants is not taken into account. Future revisions of the data that are reported by statistical agencies and available to researchers may blur the information that investors actually possessed while making decisions in the past, and hence bias a proper evaluation of the forecasts. We note in passing that all UIRP-fundamentals forecasts shown for example in Tables 1 and 2 are in this sense conducted with real-time data all along, and as such give a useful benchmark.

Unfortunately for us, the real-time data we obtained from the OECD ${ }^{21}$ starts only from February 1999, and we thus conducted our study on a shorter ${ }^{22}$ data set: February 1999-April 2017, data permitting. The results are given in Table 6 for the case where we allow asymmetric coefficients on the fundamentals. Compared to Tables 1 and 2, this points to a deterioration of the forecasting abilities of both of our machine learning methods, though we still obtain improvements in RMSE versus the random walk in the majority of the cases for PPP-and UIRP-based fundamentals. This is further confirmed in the economic evaluation criterion in Table 7 , as well as in the directional results in Table D.13 of the online supplementary material. In a separate study, shown in Tables D.19-D.24 in Appendix D of the online supplementary material, we re-ran the real-time data and revised-fundamentals forecasts on the same data period of February 1999-December 2014 (not all time-series from our original data set could be extended to 2017), with similar conclusions.

Several points need to be made here. First, as explained earlier in Section 3.2, shortening time-series in general leads to worse predictions for these machine-learning methods. The UIRP-based fundamentals are always real-time, and although we see a similar number of Theil values $<1$ for forecasts using them in comparison to what was shown in Table 1 (for the same set of currencies), these improvements are smaller (close to 1), though there is also an across-the-board performance drop in Tables 4 and 5. Second, OLS-based methods do not provide different (better) forecasting performance either. In fact, as shown in Table D.12 of the online supplementary material, machine-learning methods beat OLS-based ones in terms of RMSE improvement in a similar fashion as before (i.e., Table 3), at times spectacularly (cf. the $25.1 \%$ gain in terms of RMSE for $\mathrm{CHF} / \mathrm{USD}$ for sequential ridge regression with discount factors and all fundamentals considered). Third, using same time-period comparisons for 1999-2014 (Tables D.19-D.24 of the online supplementary material), there is no difference in performance when using real-time or revised data. This is in line with the literature, e.g., Ince (2014), which broadly concludes that the lack of use of real-time and actual data is not related to the failure of OLS-based methods. The conclusions based on these exercises have to be taken with caution, however, as they may be sample-specific. It is clear from Figures D.1-D.3 of the online supplementary material, based on the original revised-data forecasting, that after the 2008 crisis (ca. the last 80 observations) the pattern of the weights picked by the exponentially weighted average strategy with discount factors changes dramatically

\footnotetext{
${ }^{21}$ Available at http://stats . oecd.org/mei/default.asp?rev=1

${ }^{22}$ Because the period is essentially half as long as before, we also set $t_{0}=60$ months as a training period for the algorithms and as a window for rolling OLS regression, instead of the original value $t_{0}=120$ months.
} 
for the three shown currencies (also true for the others analyzed, but not shown), which could point to an important "regime" change, perhaps related to the central bank or market reactions to the crisis.

\subsection{Taylor-rule fundamentals}

A recent strand in the literature has identified Taylor-rule fundamentals as useful in achieving exchange rate predictability (which is not the same as forecastability; see Footnote 25). As Taylor-rule fundamentals can be created from the data in our possession, we also re-ran forecasting equations based on (5) with these fundamentals for our basic decoupled sample. The results are shown in Table D.25 of Appendix D of the online supplementary material.

Forecasts based on Taylor-rule model fundamentals beat the random walk in 9 of the 12 cases, no matter how we calculate the output gaps. In 6 of the 12 cases for fundamentals including output gaps - constructed either using deviations from both a linear and a quadratic trend, or from a Hodrick-Prescott filtered trend - such improvements are statistically significant at the $10 \%$ level according to the bootstrapped DM-test $p$-values. In this respect, globally they fare better than forecasts based on UIRP fundamentals (more statistically significant results) but slightly worse than predictions based on PPP fundamentals. Indeed, the Taylor-rule fundamentals as shown in (5) do include inflation differentials, precisely those used in PPP-based models - see (2) -, whereas interest rate differentials are lagged by one period. As we do not estimate models, we do not investigate further what signs the coefficients on the inflation rates have in our forecasting equations.

Also in these cases, OLS methods do not ${ }^{23}$ allow us to consistently beat the random walk. The rolling OLS regression actually achieves a forecasting accuracy that is consistently worse than the random walk (all Theil rations are $>1$, all DM test statistics are negative). As for recursive OLS regression, gains in forecasting accuracy are observed only for 3 of the $4 \times 12$ cases considered (but these 3 occurrences are all statistically significant).

\section{Related literature}

Exchange rate forecasting literature. Rossi (2013) and Della Corte and Tsiakas (2012) provide comprehensive recent reviews of the exchange-rate forecasting literature so we keep ours to a bare minimum. There have been different ways in which researchers have tried to cope with the negative result of Meese and Rogoff (1983) that showed that the simple exchange rate models from the 1970s (i.e., those that proposed the fundamentals we study here) did poorly in comparison to a random walk without drift (a no-change prediction) in forecasting exchange rates in the floating period after 1973 for short forecast horizons. One way to cope, that we have pursued here, has been to use better

\footnotetext{
${ }^{23}$ A similar remark needs to be made as in Section 5.1, see Footnote 18 therein: in many cases, especially for the rolling OLS regression, the Theil ratios are $>1$ (equivalently, the DM statistics are negative) while the bootstrapped DM $p$-values would otherwise indicate statistical significance. However, this merely means that there is no gain in forecasting accuracy in the first place, so determining whether it is significant or not is irrelevant. Technical details on why such seemingly paradoxical situations arise are provided in the mentioned footnote. Indeed, this is a documented fact in the literature.
} 
tools to extract information from the data (better forecasting tools, not necessarily statistical/econometric tools). Some of the other solutions proposed involve, for example, cointegration techniques (in Mark, 1995) combined with the use of panel data (in Mark and Sul, 2001), long samples in panels as in Rapach and Wohar (2002), and including a large set of countries (in Cerra and Saxena, 2010). These techniques are typically used for longer-horizon (over 1 year) forecasts when it is believed that the long-run relationship, as modeled by the cointegrating equations, kicks in. The cited studies obtain some success in demonstrating predictability and/or forecastability at longer horizons (typically more than 2 years). We have focused on short-term forecasts and use single-equation methods - a setup in which forecastability has not been ascertained until relatively recently. For the short-run, Greenaway-Mcgrevy et al. (2012) obtained considerable success in outpredicting the no-change prediction using factor analysis, extracting the factors from the exchange rates themselves (but not the economic fundamentals). Dal Bianco et al. (2012) obtained forecastability for the Euro/U.S. dollar rate at weekly to monthly horizons using a stylized econometric model that mixes information from different fundamentals arriving at different frequencies. Our method is more general, directly geared for prediction, and importantly, comes with certain theoretical guarantees on convergence of the RMSEs (see Section 3.2).

Sequential combination of fundamentals. The methodology used for forecasting in this paper consists of sequentially combining (aggregating) fundamentals. The distinguishing feature of our methodology is that it is sequential in nature; in contrast, all other approaches we know of that forecast exchange rates use batch estimation or learning methods that need to be run in an incremental way (which usually leads to the loss of the theoretical guarantees associated with the batch case). These approaches can be divided into two groups: (i) the learning methods of the machine-learning literature, and (ii) the estimation methods of the statistics literature.

The first group contains, for instance, the studies of Li et al. (2015) and Wright (2008) for exchange rates, and Bajari et al. (2015) for demand estimation. The first two references are perhaps the closest efforts to ours insofar as the idea of extracting information from fundamentals to forecast exchange rates is concerned. In the introduction, we have already compared our approach and results to those of $\mathrm{Li}$ et al. (2015): the final improvement in performance of end-of-month forecasts with respect to no change are comparable, though ours are slightly better. More importantly however, we obtain this improvement by considering only well-identified fundamentals (PPP or UIRP fundamentals, instead of using "kitchen-sink" aggregation) and by implementing machine-learning methods that come with theoretical performance guarantees (unlike the elastic net method used by $\mathrm{Li}$ et al. (2015)). Our approach thus conveys economic meaning and could therefore be considered "safer". Wright (2008) used a method called Bayesian model averaging. The building blocks for prediction he uses are a large number of predictors, many of which do not come from the standard models considered in this paper (not only classic fundamentals are used). His approach does not give improved forecasts compared to the no-change prediction in a statistically significant way in most cases he considers. Moreover, he does not explain how to properly choose the "shrinkage" parameter that retains the informativeness of priors without knowing the properties of the data ex ante. Our 
methods, in contrast, are entirely data-driven ${ }^{24}$.

As for estimation methods, two approaches related to ours are estimation of nonlinear models, and models with time-varying parameters. Rossi (2013) states that various nonlinear methods were not particularly successful in forecasting exchange rates, while Rossi (2006) questions the robustness of time-varying parameter models. Bacchetta et al. (2010) argue that the gain from using such an approach would be minimal in practice. On simulated data, these authors find that the benefits from using such models in terms of greater explanatory power are in practice outweighed by additional estimation errors in the time-varying parameters. Schinasi and Swamy (1989) reassess the study of Meese and Rogoff (1983) using various nonlinear methods, including an early version of ridge regression. Engel (1994) documents the failure of a Markov-switching model to beat the no-change prediction in forecasting.

Other issues considered in exchange rate forecasting. Another way that researchers have tried to improve the ability to forecast exchange rates from fundamentals is to consider different economic models with other fundamentals. It has been seen recently that exchange rate models based on Taylor-rule fundamentals perform well in ascertaining the predictability ${ }^{25}$ of exchange rates at short horizons (see Engel and West, 2006, Molodtsova and Papell, 2009, Molodtsova et al., 2008, Giacomini and Rossi, 2010, Molodtsova et al., 2011, Rossi and Inoue, 2012; though Rogoff and Stavrakeva, 2008 disagree) but do not find that these perform much better in terms of forecasting. For this reason we test our methods on Taylor-rule fundamentals, obtaining good forecasting results - only the plain vanilla inflation rates used as fundamentals work better (see Section 5.6).

Another successful fundamental was the behavior of net foreign assets, as in Gourinchas and Rey (2007) and Della Corte et al. (2012). The fundamentals to conduct these tests are available at 3-month frequencies, resulting in fewer observations that can be used, so we did not investigate them here. Other studies have assessed the forecasting ability of exchange rate models of the 1990s, including Cheung et al. (2005), differences in the term structures of forward premia (Clarida et al., 2003), and the scapegoat model (Fratzscher et al., 2015). Given the scope of our exercise and the gaps in some necessary data, we did not evaluate these models with our machine-learning methods, but this may be a useful research agenda for the future.

\footnotetext{
${ }^{24}$ In fact, some inspiration could be taken from them to perform a data-driven choice of the "shrinkage" parameter of Wright (2008).

25 Predictability is a different concept to forecastability. In Molodtsova and Papell (2009), it means testing whether the estimated coefficients of a model are jointly significantly different from zero when explaining changes in the exchange rate. It does not mean that a model that exhibits predictability necessarily provides better forecasts (in the literature, typically it does not). In general, the focus of these and many other attempts is rather to assess whether fundamentals play a role in exchange rate determination, since it can be motivated theoretically why the forecasts they produce in terms of an evaluation criterion such as RMSE may fare worse than those of a forecast based on no change in the exchange rate. (See Rossi, 2013 for a discussion of this. Special tests were designed by West, 1996 and Clark and West, 2006, 2007 for this purpose. See also Appendix B.3 of the online supplementary material.) In this paper, however, we are not focused on predictability - we actually are interested in whether we can produce better forecasts of exchange rates than the no-change one. We are also not interested in validating a particular model - that is, trying to fit coefficients and check whether the signs and magnitudes are those posited by the theory; we simply try to extract from the fundamentals in question information useful for the behavior of exchange rates.
} 


\section{Conclusions}

In this paper we have applied methods stemming from the field of machine learning sequential ridge regression with discount factors and the exponentially weighted average strategy with discount factors - to the perennial problem of exchange rate forecasting. In doing so, we obtain gains in forecasting in terms of the standard RMSE criterion for PPP or UIRP fundamentals that were not found using traditionally applied estimation methods based on OLS regressions. The key is to use these machine-learning forecasting schemes to do what they are good for: produce forecasts - and not try to estimate some underlying model (if any such model exists) as has traditionally been the case with more statistical methods. We conclude thus that a major problem in international economics - whether there is a short-term relationship between "classic" fundamentals and exchange rates that can be detected and that beats the random walk - is answered in the affirmative under the condition that proper machine-learning techniques, e.g., sequential ridge regression with discount factors, or the exponentially weighted average strategy with discount factors, are applied. Our success points to the potential of such techniques for improving the evaluation of economic problems.

Machine learning techniques also serve to effectively aggregate information from many sources. A tempting exercise, beyond the scope of this paper, is to evaluate the forecasting performance when including many more fundamentals than the "classic" ones considered here that come from suggestions in the literature - for example those based on productivity, interest rate yield curves, net foreign assets, etc. One set of fundamentals - based on the UIRP relationship - is especially promising since interest rates (for various maturities) can be obtained for long-time periods at high frequencies. Venturing further, one could consider many more time-series that are not typically associated with exchange rate forecasting, in the true spirit of machine learning.

As with any new method applied to exchange rate forecasting, it remains to be seen whether our results can be replicated for other currencies, samples, forecasting periods and fundamentals. However, given the robustness of the results shown in this paper, we hope that the application of these methods to exchange rate forecasting will stand the test of time and allow for better predictions and decision-making in the future.

\section{Acknowledgements}

This work was supported by Investissements d'Avenir (grant number ANR-11-IDEX0003/Labex Ecodec/ANR-11-LABX-0047).

\section{References}

Alvarez, F., Atkeson, A., Kehoe, P. J., 2007. If exchange rates are random walks, then almost everything we say about monetary policy is wrong. American Economic Review 97, 339-345.

Amat, C., Michalski, T., Stoltz, G., 2018a. Data set associated with the article "Fundamentals and exchange rate forecastability with simple machine learning methods". Posted on Mendeley.com.

URL http://dx.doi.org/10.17632/yxystdn2hz.1 
Amat, C., Michalski, T., Stoltz, G., 2018b. Fundamentals and exchange rate forecastability with simple machine learning methods. Supplementary material.

URL https://halshs.archives-ouvertes.fr/halshs-01003914

Auer, P., Cesa-Bianchi, N., Gentile, C., 2002. Adaptive and self-confident on-line learning algorithms. Journal of Computer and System Sciences 64, 48-75.

Azoury, K. S., Warmuth, M., 2001. Relative loss bounds for on-line density estimation with the exponential family of distributions. Machine Learning 43, 211-246.

Bacchetta, P., van Wincoop, E., 2004. A scapegoat model of exchange-rate fluctuations. American Economic Review 94, 114-118.

Bacchetta, P., van Wincoop, E., Beutler, T., June 2010. Can parameter instability explain the Meese-Rogoff puzzle? In: NBER International Seminar on Macroeconomics 2009. University of Chicago Press, pp. 125-173.

URL http://www. nber.org/chapters/c11912

Bajari, P., Nekipelov, D., Ryan, S. P., Yang, M., 2015. Demand estimation with machine learning and model combination. NBER Working paper 20955.

Boyd, S., Vandenberghe, L., 2009. Convex Optimization, 2nd Edition. Cambridge University Press.

Cerra, V., Saxena, S. C., 2010. The monetary model strikes back: Evidence from the world. Journal of International Economics 81, 184-196.

Cesa-Bianchi, N., 1999. Analysis of two gradient-based algorithms for on-line regression. Journal of Computer and System Sciences 59 (3), 392-411.

Cesa-Bianchi, N., Freund, Y., Haussler, D., Helmbold, D. P., Schapire, R., Warmuth, M., 1997. How to use expert advice. Journal of the ACM 44 (3), 427-485.

Cesa-Bianchi, N., Lugosi, G., 2006. Prediction, Learning, and Games. Cambridge University Press.

Cheung, Y.-W., Chinn, M. D., Garcia Pascual, A., 2005. Empirical exchange rate models of the nineties: Are any fit to survive? Journal of International Money and Finance $24,1150-1175$.

Clarida, R. H., Sarno, L., Taylor, M. P., Valente, G., 2003. The out-of-sample success of term structure models: A step beyond. Journal of International Economics 60, 61-83.

Clark, T. E., West, K. D., 2006. Using out-of-sample mean squared prediction errors to test the martingale difference hypothesis. Journal of Econometrics 135 (1-2), 155-186.

Clark, T. E., West, K. D., 2007. Approximately normal tests for equal predictive accuracy in nested models. Journal of Econometrics 138, 291-311.

Dal Bianco, M., Camacho, M., Perez Quiros, G., 2012. Short-run forecasting of the EuroDollar exchange rate with economic fundamentals. Journal of International Money and Finance 31, 377-396. 
Debry, E., Mallet, V., 2014. Ensemble forecasting with machine learning algorithms for ozone, nitrogen dioxide and PM10 on the Prev'Air platform. Atmospheric Environment 91, 71-84.

Della Corte, P., Sarno, L., Sestieri, G., 2012. The predictive information content of external imbalances for exchange rate returns: How much is it worth? The Review of Economics and Statistics 94, 100-115.

Della Corte, P., Sarno, L., Tsiakas, I., 2009. An economic evaluation of empirical exchange rate models. Review of Financial Studies 22, 3491-3530.

Della Corte, P., Sarno, L., Tsiakas, I., 2011. Spot and forward volatility in foreign exchange. Journal of Financial Economics 100, 496-513.

Della Corte, P., Tsiakas, I., 2012. Statistical and economic methods for evaluating exchange rate predictability. In: James, J., Marsh, I. W., Sarno, L. (Eds.), Handbook of Exchange Rates. Wiley, pp. 221-263.

Devaine, M., Gaillard, P., Goude, Y., Stoltz, G., 2013. Forecasting the electricity consumption by aggregation of specialized experts; application to Slovakian and French country-wide (half-)hourly predictions. Machine Learning 90(2), 231-260.

Diebold, F. X., 2012. Comparing predictive accuracy, twenty years later: a personal perspective on the use and abuse of Diebold-Mariano tests. NBER Working paper 18391.

Diebold, F. X., Mariano, R. S., 1995. Comparing predictive accuracy. Journal of Business and Economic Statistics 13 (3), 253-263.

Engel, C., 1994. Can the Markov switching model forecast exchange rates? Journal of International Economics 36, 151-165.

Engel, C., West, K. D., 2005. Exchange rates and fundamentals. Journal of Political Economy 113, 485-517.

Engel, C., West, K. D., 2006. Taylor rules and the Deutschmark-Dollar real exchange rate. Journal of Money, Credit and Banking 38 (5), 1175-1194.

Fratzscher, M., Rime, D., Sarno, L., Zinna, G., 2015. The scapegoat theory of exchange rates: the first tests. Journal of Monetary Economics 70, 1-21.

Gaillard, P., Goude, Y., 2015. Forecasting the electricity consumption by aggregating experts; how to design a good set of experts. In: Antoniadis, A., Brossat, X., Poggi, J.-M. (Eds.), Modeling and Stochastic Learning for Forecasting in High Dimension. Lecture Notes in Statistics. Springer, to appear.

Gerchinovitz, S., 2011. Prediction of individual sequences and prediction in the statistical framework: some links around sparse regression and aggregation techniques. Ph.D. thesis, Université Paris-Sud, Orsay.

Giacomini, R., Rossi, B., 2010. Forecast comparisons in unstable environments. Journal of Applied Econometrics 25 (4), 595-620. 
Gourinchas, P.-O., Rey, H., 2007. International financial adjustment. Journal of Political Economy 115 (4), 665-703.

Greenaway-Mcgrevy, R., Mark, N. C., Sul, D., Wu, J.-L., 2012. Exchange rates as exchange rate common factors. Working paper.

Hoerl, A. E., Kennard, R. W., 1970. Ridge regression: Biased estimation for nonorthogonal problems. Technometrics 12, 55-67.

Ince, O., 2014. Forecasting exchange rates out-of-sample with panel methods and realtime data. Journal of International Money and Finance 43, 1-18.

Li, J., Tsiakas, I., Wang, W., 2015. Predicting exchange rates out of sample: can economic fundamentals beat the random walk? Journal of Financial Econometrics 13 (2), 293341 .

Littlestone, N., Warmuth, M., 1994. The weighted majority algorithm. Information and Computation 108, 212-261.

Mallet, V., 2010. Ensemble forecast of analyses: Coupling data assimilation and sequential aggregation. Journal of Geophysical Research 115, D24303.

Mallet, V., Mauricette, B., Stoltz, G., 2007. Description of sequential aggregation methods and their performances for ozone ensemble forecasting. Technical report DMA07-08, École normale supérieure, Paris, available at ftp://ftp.dma.ens.fr/pub/ reports/dma-07-08.pdf.

Mark, N. C., 1995. Exchange rates and fundamentals: Evidence on long-horizon predictability. American Economic Review 85, 201-218.

Mark, N. C., Sul, D., 2001. Nominal exchange rates and monetary fundamentals: Evidence from a small post-Bretton Woods panel. Journal of International Economics 53 29-52.

Mauricette, B., Mallet, V., Stoltz, G., 2009. Ozone ensemble forecast with machine learning algorithms. Journal of Geophysical Research 114, D05307.

Meese, R. A., Rogoff, K., 1983. Empirical exchange rate models of the seventies. Do they fit out of sample? Journal of International Economics 14, 3-24.

Molodtsova, T., Nikolsko-Rzhevskyy, A., Papell, D. H., 2008. Taylor rules with real-time data: A tale of two countries and one exchange rate. Journal of Monetary Economics 55, S63-S79.

Molodtsova, T., Nikolsko-Rzhevskyy, A., Papell, D. H., March-April 2011. Taylor Rules and the Euro. Journal of Money, Credit and Banking 43 (2-3), 535-552.

Molodtsova, T., Papell, D. H., 2009. Out-of-sample exchange rate predictability with Taylor rule fundamentals. Journal of International Economics 77, 167-180.

Plakandaras, V., Papadimitriou, T., Gogas, P., 2015. Forecasting daily and monthly exchange rates with machine learning techniques. Journal of Forecasting 34, 560-573. 
Rapach, D. E., Wohar, M. E., 2002. Testing the monetary model of exchange rate determination: new evidence from a century of data. Journal of International Economics $58,359-385$.

Ravn, M., Uhlig, H., 2002. On adjusting the Hodrick-Prescott filter for the frequency of observations. The Review of Economics and Statistics 84, 371-375.

Rogoff, K. S., Stavrakeva, V., 2008. The continuing puzzle of short horizon exchange rate forecasting. Working Paper 14071, National Bureau of Economic Research.

Rossi, B., 2006. Are exchange rates really random walks? Some evidence robust to parameter instability. Macroeconomic Dynamics 10, 20-38.

Rossi, B., 2013. Exchange rate predictability. Journal of Economic Literature 51, 1063 1119 .

Rossi, B., Inoue, A., 2012. Out-of-sample forecast tests robust to the choice of window size. Journal of Business and Economic Statistics 30, 432-453.

Schinasi, G. J., Swamy, P. A. V. B., 1989. The out-of-sample forecasting performance of exchange rate models when coefficients are allowed to change. Journal of International Money and Finance 8, 375-390.

Stoltz, G., 2010. Agrégation séquentielle de prédicteurs : méthodologie générale et applications à la prévision de la qualité de l'air et à celle de la consommation électrique. Journal de la Société Française de Statistique 151 (2), 66-106.

Vovk, V., 1990. Aggregating strategies. In: Proceedings of the Third Annual Workshop on Computational Learning Theory (COLT). pp. 372-383.

Vovk, V., 2001. Competitive on-line statistics. International Statistical Review 69, 213248.

West, K. D., 1996. Asymptotic inference about predictive ability. Econometrica 64, 10671084 .

West, K. D., 2006. Forecast evaluation. In: Elliott, G., Granger, C. W., Timmermann, A. (Eds.), Handbook of Economic Forecasting. Vol. 1. Elsevier, pp. 99-134.

West, K. D., Edison, H. J., Dongchul, C., 1993. A utility based comparison of some models of exchange rate volatility. Journal of International Economics 35, 23-45.

Wright, J. H., 2008. Bayesian Model Averaging and exchange rate forecasts. Journal of Econometrics 146, 329-341. 
Table 1: 1-month ahead forecasts for the PPP, UIRP, monetary model and all fundamentals: decoupled formulation.

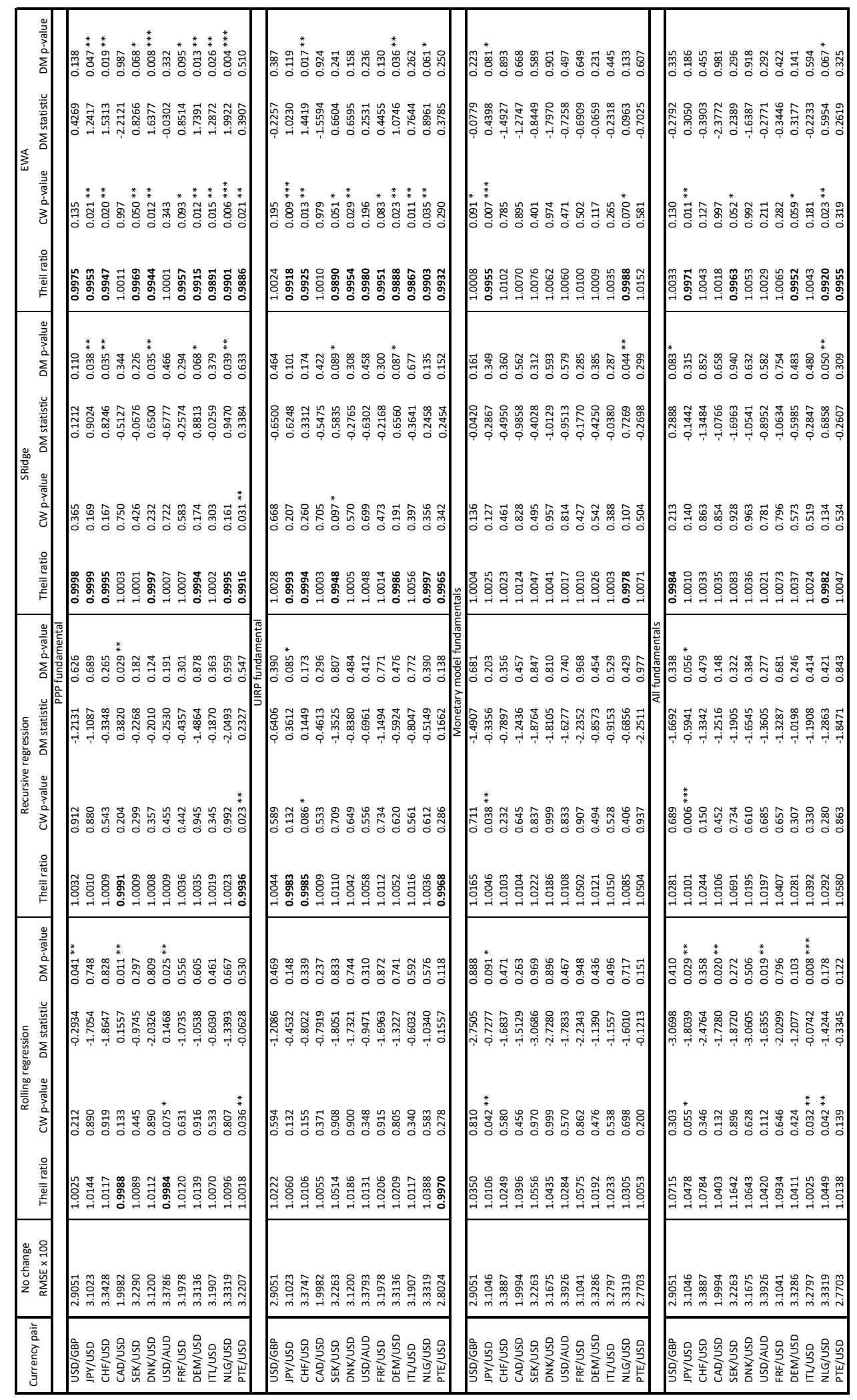

Notes: This table presents the results of forecasting end-of-month exchange rate 1-month ahead by the no-change prediction, the rolling OLS, recursive OLS, SRidge and EWA methods based on decoupled fundamentals. Column 1 shows the RMSE values for the no-change prediction. For each method, the Theil ratio (RMSE of the given method / RMSE of the no-change prediction), the CW p-values, the DM statistic and its bootstrapped p-value are shown. The CW and DM tests are one-sided tests of equal outof-sample prediction accuracy $\left(H_{0}\right)$ against superior out-of-sample prediction accuracy $\left(H_{1}\right)$ for the methods considered compared to the no-change prediction. ${ }^{* *},{ }^{* *}$, and $*$ denote statistical significance at the $1 \%, 5 \%$, and $10 \%$ levels. Theil ratios $<1$ are indicated in bold. Sample period for the exchange rates: March 1973 - December 2014 unless shorter (indicated in Appendix A of the online supplementary material). 
Table 2: 1-month ahead forecasts for the PPP, UIRP, monetary model and all fundamentals: coupled formulation.

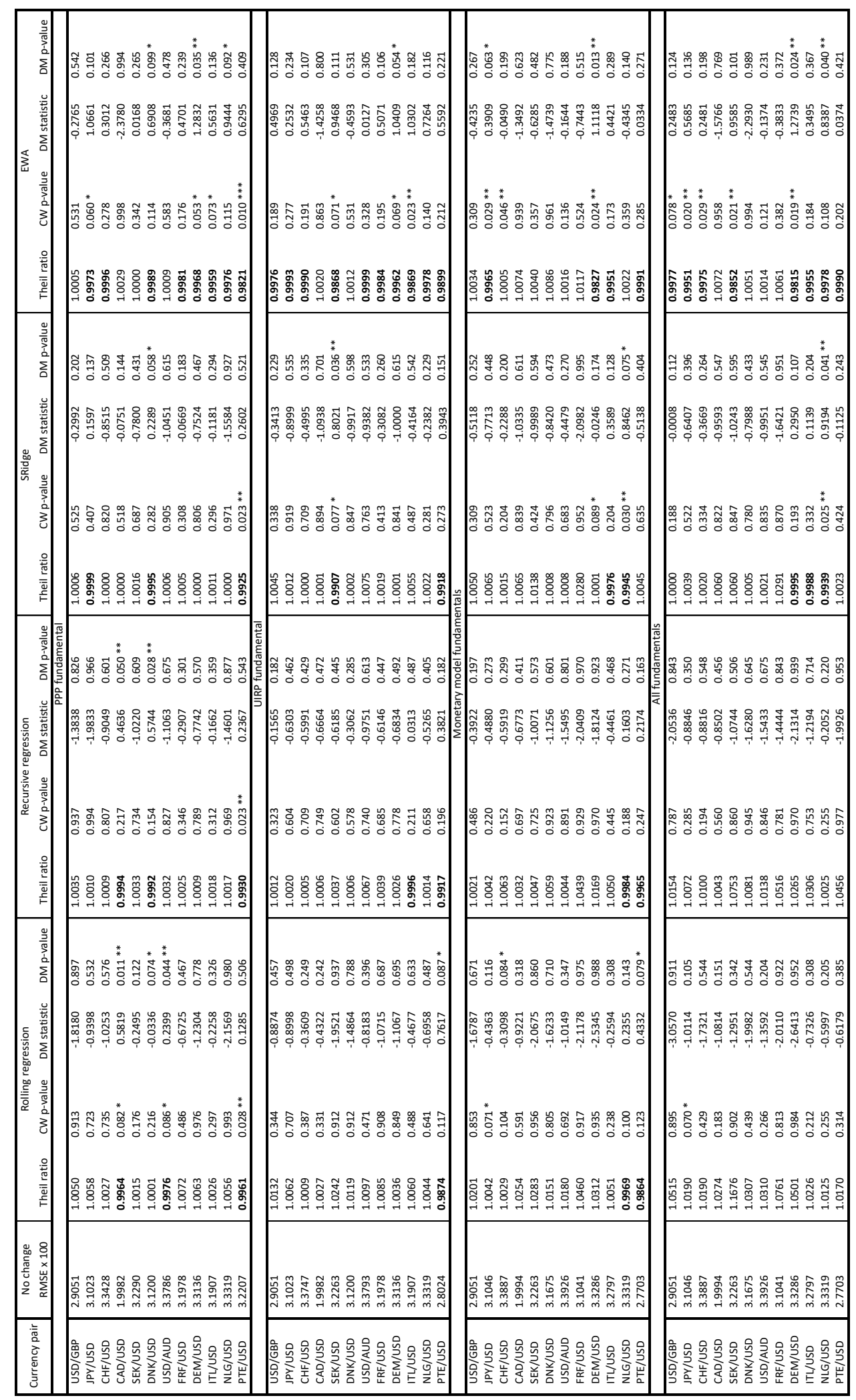

Notes: This table presents the results of forecasting end-of-month exchange rate 1-month ahead by the no-change prediction, the rolling OLS, recursive OLS, SRidge and EWA methods based on coupled fundamentals. Column 1 shows the RMSE values for the no-change prediction. For each method, the Theil ratio (RMSE of the given method / RMSE of the no-change prediction), the CW p-values, the DM statistic and its bootstrapped p-value are shown. The CW and DM tests are one-sided tests of equal outof-sample prediction accuracy $\left(H_{0}\right)$ against superior out-of-sample prediction accuracy $\left(H_{1}\right)$ for the methods considered compared to the no-change prediction. ${ }^{* *},{ }^{* *}$, and $*$ denote statistical significance at the $1 \%, 5 \%$, and $10 \%$ levels. Theil ratios $<1$ are indicated in bold. Sample period for the exchange rates: March 1973 - December 2014 unless shorter (indicated in Appendix A of the online supplementary material). 


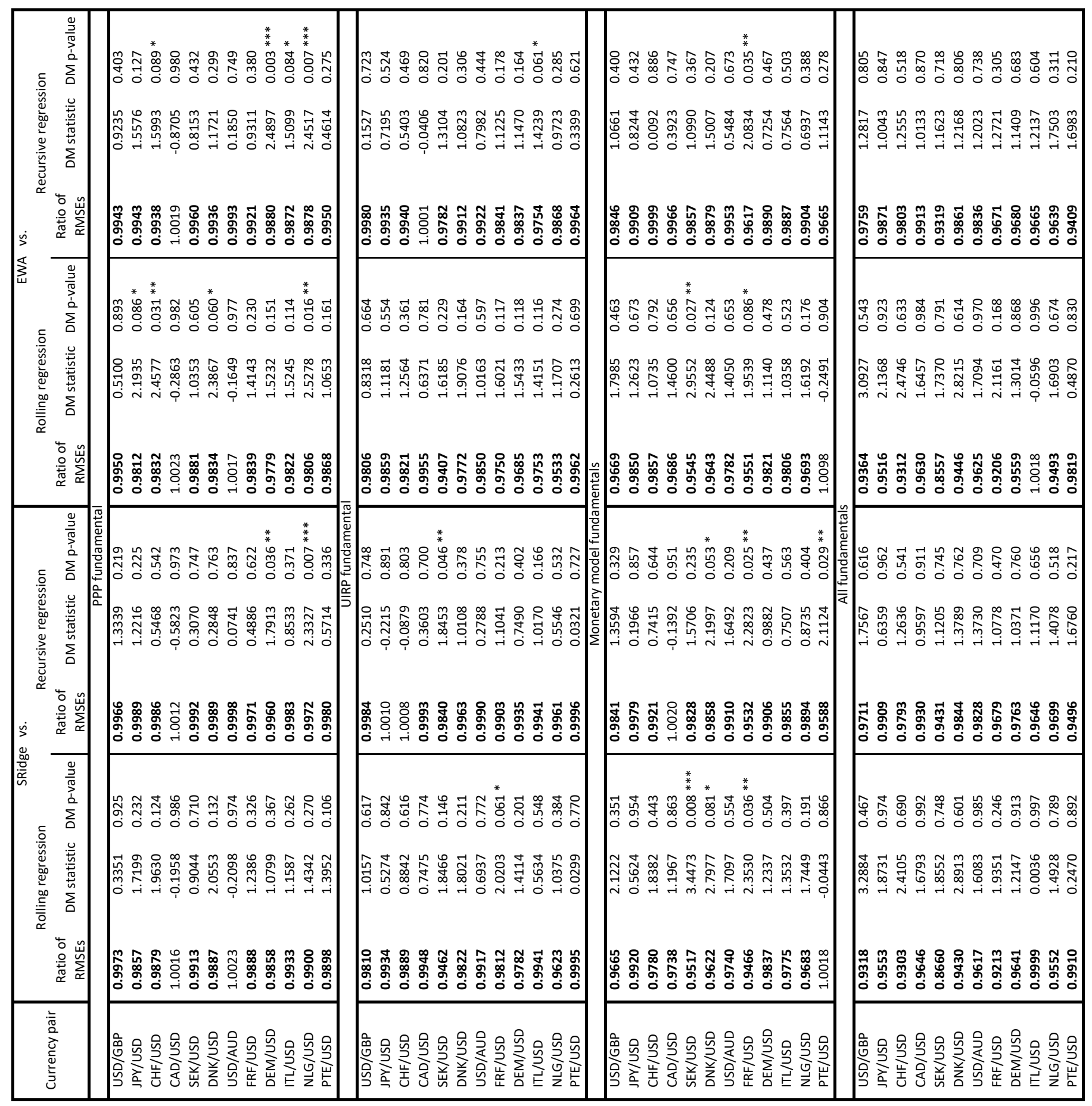

Notes: This table presents the comparison of forecasting performance between, respectively, SRidge and EWA vs. the rolling and recursive OLS based on decoupled fundamentals. Columns 1-6 show the comparison between SRidge and OLS methods, while columns 7-12 for EWA vs. OLS. For each comparison, the Theil ratio (RMSE of the given method / RMSE of the compared) and the DM statistic and its bootstrapped p-value are shown. The DM test is a one-sided test of equal out-of-sample prediction accuracy $\left(H_{0}\right)$ against superior out-of-sample prediction accuracy $\left(H_{1}\right)$ of the machine-learning method considered against the OLS methods. ***, **, and * denote statistical significance at the $1 \%, 5 \%$, and $10 \%$ levels. Theil ratios $<1$ are indicated in bold. Sample period for the exchange rates: March 1973 - December 2014 unless shorter (indicated in Appendix A of the online supplementary material). 
Table 4: Directional predictions of the exchange rates based on decoupled fundamentals.

\begin{tabular}{|c|c|c|c|c|c|c|c|c|c|c|c|c|}
\hline \multirow[b]{2}{*}{ Currency pair } & \multicolumn{3}{|c|}{ Rolling regression } & \multicolumn{3}{|c|}{ Recursive regression } & \multicolumn{3}{|c|}{ Sridge } & \multicolumn{3}{|c|}{ EWA } \\
\hline & $\begin{array}{c}\text { Proportion of } \\
\text { changes } \\
\text { predicted }\end{array}$ & DM statistic & DM p-value & $\begin{array}{c}\text { Proportion of } \\
\text { changes } \\
\text { predicted }\end{array}$ & DM statistic & DM p-value & $\begin{array}{c}\text { Proportion of } \\
\text { changes } \\
\text { predicted }\end{array}$ & DM statistic & DM p-value & $\begin{array}{l}\text { Proportion of } \\
\text { changes } \\
\text { predicted }\end{array}$ & DM statistic & DM p-value \\
\hline \multicolumn{13}{|c|}{ PPP fundamental } \\
\hline USD/GBP & 0.512 & 0.4294 & 0.300 & 0.493 & -0.2498 & 0.593 & 0.470 & -0.9744 & 0.829 & 0.522 & 0.6041 & 0.249 \\
\hline JPY/USD & 0.470 & -1.1431 & 0.860 & 0.462 & -1.3166 & 0.906 & 0.546 & 1.7192 & $0.017^{* *}$ & 0.543 & 1.4995 & $0.027 * *$ \\
\hline CHF/USD & 0.525 & 0.8142 & 0.165 & 0.488 & -0.3462 & 0.637 & 0.556 & 1.9711 & $0.011 * *$ & 0.541 & 1.3711 & $0.064 *$ \\
\hline CAD/USD & 0.528 & 0.9373 & 0.138 & 0.496 & -0.1207 & 0.554 & 0.496 & -0.1153 & 0.516 & 0.441 & -2.2202 & 0.989 \\
\hline SEK/USD & 0.501 & 0.0410 & 0.459 & 0.480 & -0.5793 & 0.718 & 0.493 & -0.1838 & 0.547 & 0.512 & 0.4047 & 0.314 \\
\hline DNK/USD & 0.499 & -0.0424 & 0.495 & 0.525 & 0.6536 & 0.223 & 0.564 & 2.1772 & $0.010^{* * *}$ & 0.559 & 2.2801 & $0.004^{* * *}$ \\
\hline USD/AUD & 0.494 & -0.1572 & 0.491 & 0.497 & -0.0741 & 0.475 & 0.483 & -0.5116 & 0.657 & 0.480 & -0.6659 & 0.725 \\
\hline FRF/USD & 0.481 & -0.3854 & 0.655 & 0.540 & 0.6175 & 0.248 & 0.481 & -0.4106 & 0.679 & 0.571 & 1.8923 & $0.028 * *$ \\
\hline DEM/USD & 0.455 & -1.0227 & 0.885 & 0.481 & -0.4448 & 0.728 & 0.603 & 2.3448 & $0.020 * *$ & 0.608 & 2.5567 & $0.008 * * *$ \\
\hline ITL/USD & 0.487 & -0.2303 & 0.597 & 0.466 & -0.6356 & 0.745 & 0.466 & -0.6356 & 0.755 & 0.577 & 1.3190 & $0.089 *$ \\
\hline NLG/USD & 0.519 & 0.3670 & 0.397 & 0.455 & -0.9079 & 0.833 & 0.587 & 1.9547 & $0.040 * *$ & 0.593 & 1.9582 & $0.055^{*}$ \\
\hline PTE/USD & 0.481 & -0.2859 & 0.777 & 0.497 & -0.0432 & 0.680 & 0.497 & -0.0432 & 0.702 & 0.550 & 0.9484 & 0.290 \\
\hline \multicolumn{13}{|c|}{ UIRP fundamental } \\
\hline USD/GBP & 0.496 & -0.1160 & 0.531 & 0.522 & 0.7841 & 0.177 & 0.480 & -0.6037 & 0.733 & 0.517 & 0.5310 & 0.268 \\
\hline JPY/USD & 0.546 & 1.4607 & $0.037^{* *}$ & 0.522 & 0.7917 & 0.168 & 0.562 & 2.4264 & $0.003 * * *$ & 0.570 & 2.7419 & $0.001 * * *$ \\
\hline CHF/USD & 0.566 & 2.4290 & $0.011 * *$ & 0.547 & 1.7035 & $0.044 * *$ & 0.550 & 1.7495 & $0.033 * *$ & 0.563 & 2.0034 & $0.006 * * *$ \\
\hline CAD/USD & 0.551 & 1.9736 & $0.015^{* *}$ & 0.522 & 0.7710 & 0.202 & 0.493 & -0.1882 & 0.583 & 0.483 & -0.5147 & 0.651 \\
\hline SEK/USD & 0.497 & -0.0855 & 0.504 & 0.471 & -0.9192 & 0.839 & 0.508 & 0.2847 & 0.346 & 0.539 & 1.4367 & $0.062 *$ \\
\hline DNK/USD & 0.501 & 0.0427 & 0.486 & 0.483 & -0.4942 & 0.685 & 0.512 & 0.4001 & 0.328 & 0.564 & 2.5218 & $0.002 * * *$ \\
\hline USD/AUD & 0.500 & 0.0000 & 0.424 & 0.514 & 0.4352 & 0.287 & 0.470 & -0.9811 & 0.792 & 0.492 & -0.2896 & 0.397 \\
\hline FRF/USD & 0.497 & -0.0413 & 0.494 & 0.460 & -0.7051 & 0.772 & 0.561 & 1.6855 & $0.029 * *$ & 0.593 & 2.5907 & $0.002 * * *$ \\
\hline DEM/USD & 0.508 & 0.1344 & 0.599 & 0.540 & 0.8111 & 0.368 & 0.593 & 1.9107 & $0.095 *$ & 0.608 & 2.5974 & $0.007 * * *$ \\
\hline ITL/USD & 0.497 & -0.0470 & 0.506 & 0.466 & -0.6481 & 0.762 & 0.450 & -0.9429 & 0.855 & 0.603 & 2.1527 & $0.012 * *$ \\
\hline NLG/USD & 0.556 & 1.2824 & 0.126 & 0.561 & 1.4899 & $0.100 *$ & 0.593 & 2.1242 & $0.032 * *$ & 0.593 & 2.1898 & $0.013^{* *}$ \\
\hline PTE/USD & 0.479 & -0.3564 & 0.612 & 0.437 & -0.8302 & 0.772 & 0.437 & -1.0768 & 0.841 & 0.507 & 0.1187 & 0.383 \\
\hline \multicolumn{13}{|c|}{ Monetary model fundamentals } \\
\hline USD/GBP & 0.501 & 0.0512 & 0.461 & 0.535 & 1.0881 & 0.128 & \begin{tabular}{|l|}
0.509 \\
\end{tabular} & 0.2975 & 0.366 & 0.517 & 0.4324 & 0.247 \\
\hline JPY/USD & 0.532 & 1.1515 & $0.093 *$ & 0.524 & 0.7503 & 0.208 & 0.542 & 1.3905 & $0.067 *$ & 0.558 & 2.1103 & $0.008^{* * *}$ \\
\hline CHF/USD & 0.497 & -0.0913 & 0.536 & 0.541 & 1.4121 & $0.060 *$ & 0.541 & 1.3316 & $0.073 *$ & 0.533 & 1.2019 & $0.084 *$ \\
\hline CAD/USD & 0.513 & 0.5132 & 0.274 & 0.489 & -0.3737 & 0.626 & 0.487 & -0.4104 & 0.642 & 0.487 & -0.4902 & 0.674 \\
\hline SEK/USD & 0.432 & -2.4520 & 0.992 & 0.474 & -0.7033 & 0.772 & 0.537 & 1.4292 & $0.062 *$ & 0.524 & 0.7452 & 0.326 \\
\hline DNK/USD & 0.437 & -2.0040 & 0.984 & 0.480 & -0.5277 & 0.687 & 0.480 & -0.5528 & 0.733 & 0.489 & -0.3536 & 0.696 \\
\hline USD/AUD & 0.526 & 0.8000 & 0.154 & 0.489 & -0.4265 & 0.644 & 0.477 & -0.6859 & 0.732 & 0.497 & -0.0933 & 0.478 \\
\hline FRF/USD & 0.525 & 0.4293 & 0.300 & 0.467 & -0.5844 & 0.748 & 0.558 & 1.2868 & $0.052 *$ & 0.550 & 1.1010 & $0.085 *$ \\
\hline DEM/USD & 0.559 & 1.5897 & $0.072 *$ & 0.576 & 1.8096 & $0.058 *$ & 0.559 & 1.0695 & 0.217 & 0.548 & 1.2069 & $0.057 *$ \\
\hline ITL/USD & 0.449 & -0.8892 & 0.841 & 0.442 & -1.1587 & 0.897 & 0.513 & 0.2405 & 0.406 & 0.538 & 0.8534 & 0.118 \\
\hline NLG/USD & 0.434 & -1.2346 & 0.945 & 0.513 & 0.2685 & 0.461 & 0.582 & 1.7186 & $0.078 *$ & 0.534 & 0.9146 & 0.140 \\
\hline PTE/USD & 0.457 & -0.5744 & 0.748 & 0.386 & -1.9096 & 0.978 & 0.371 & -2.1989 & 0.982 & 0.471 & -0.4383 & 0.468 \\
\hline \multicolumn{13}{|c|}{ All fundamentals } \\
\hline USD/GBP & 0.556 & 2.0805 & $0.011^{* *}$ & 0.491 & -0.3228 & 0.637 & 0.496 & -0.1258 & 0.514 & 0.488 & -0.3126 & 0.596 \\
\hline JPY/USD & 0.537 & 1.4172 & $0.079 *$ & 0.537 & 1.0926 & 0.147 & 0.566 & 2.1856 & $0.010 * * *$ & 0.558 & 2.2724 & $0.006 * * *$ \\
\hline CHF/USD & 0.525 & 0.8156 & 0.341 & 0.514 & 0.4455 & 0.535 & 0.541 & 1.3797 & $0.054^{*}$ & 0.547 & 1.5987 & $0.027 * *$ \\
\hline CAD/USD & 0.558 & 1.8536 & $0.036^{* *}$ & 0.503 & 0.0918 & 0.534 & 0.495 & -0.1529 & 0.570 & 0.455 & -1.4481 & 0.924 \\
\hline SEK/USD & 0.505 & 0.1815 & 0.465 & 0.495 & -0.1603 & 0.582 & 0.487 & -0.3850 & 0.620 & 0.542 & 1.6474 & $0.072 *$ \\
\hline DNK/USD & 0.529 & 0.8728 & 0.169 & 0.511 & 0.3648 & 0.352 & 0.443 & -1.6257 & 0.957 & 0.483 & -0.5244 & 0.718 \\
\hline USD/AUD & 0.517 & 0.5688 & 0.283 & 0.520 & 0.6296 & 0.293 & 0.472 & -0.8429 & 0.758 & 0.500 & 0.0000 & 0.310 \\
\hline FRF/USD & 0.567 & 1.4214 & $0.092 *$ & 0.550 & 0.9472 & 0.217 & 0.500 & 0.0000 & 0.484 & 0.533 & 0.7098 & 0.235 \\
\hline DEM/USD & 0.537 & 0.7360 & 0.310 & 0.508 & 0.1919 & 0.562 & 0.542 & 1.0706 & 0.267 & 0.576 & 1.5195 & $0.062 *$ \\
\hline ITL/USD & 0.513 & 0.2581 & 0.424 & 0.513 & 0.2700 & 0.408 & 0.429 & -1.2882 & 0.915 & 0.558 & 1.4508 & $0.027^{* *}$ \\
\hline NLG/USD & 0.513 & 0.2924 & 0.460 & 0.540 & 0.9389 & 0.213 & 0.582 & 1.7087 & $0.072 *$ & 0.561 & 1.2465 & $0.098 *$ \\
\hline PTE/USD & 0.486 & -0.1967 & 0.607 & 0.414 & -1.3264 & 0.925 & 0.471 & -0.4789 & 0.658 & 0.471 & -0.4730 & 0.666 \\
\hline
\end{tabular}

Notes: This table presents the results of forecasting the direction of change of the end-of-month exchange rate 1-month ahead by the rolling OLS, recursive OLS, SRidge and EWA methods based on decoupled fundamentals. For each method, the share of correctly forecasted changes, the DM statistic and its bootstrapped p-value are shown. The DM tests is a one-sided test of equal out-of-sample prediction accuracy $\left(H_{0}\right)$ against superior out-of-sample prediction accuracy $\left(H_{1}\right)$ for the methods considered against the benchmark of a $50 \%$ success rate. ${ }^{* * *},{ }^{* *}$, and ${ }^{*}$ denote statistical significance at the $1 \%, 5 \%$, and $10 \%$ levels. Shares $>0.5$ are indicated in bold. Sample period for the exchange rates: March 1973 - December 2014 unless shorter (indicated in Appendix A of the online supplementary material). 
Table 5: Economic criterion for evaluating forecasts for the EWA algorithm based on decoupled fundamentals.

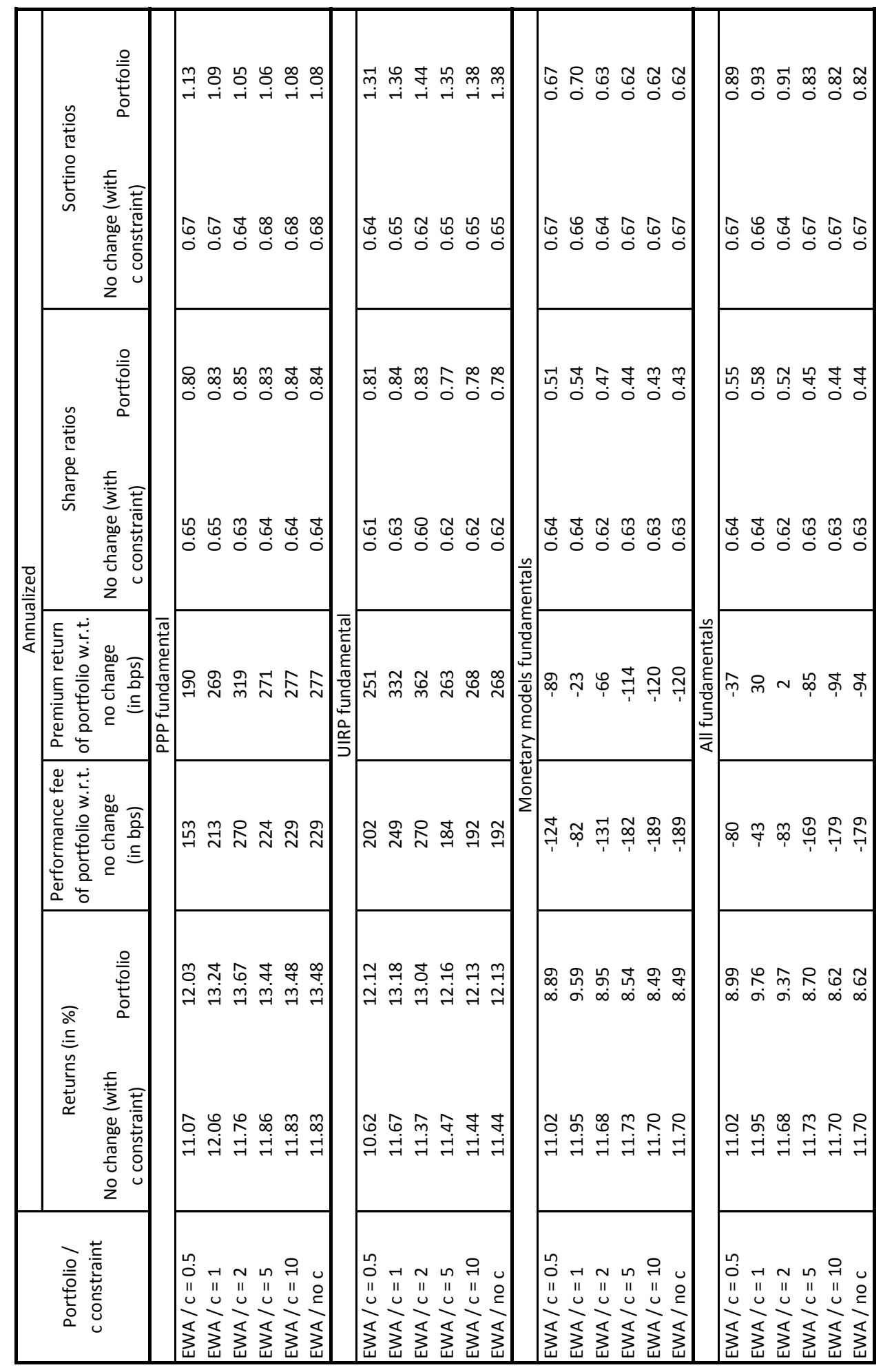

Notes: This table presents the comparison of performance of portfolios formed using forecasts from the EWA algorithm and the no-change prediction based on decoupled fundamentals, using the procedure described in Section 5.4. Column 1 gives the conditions under which the portfolios were constructed. In columns 2 and 3 the annualized returns are given for the EWA and no-change prediction-based portfolios respectively. Column 4 shows the performance fee in basis points (bps) while column 5 the premium return (in bps) of the EWA-based portfolios relative to the ones formed based on the no-change forecast. The Sharpe and Sortino ratios for portfolios created using the two competing forecasting methods are reported in columns 6-10. For details on how these portfolios were created please consult Appendix C.3 of the online supplementary material. The results for the portfolios based on the no-change exchange rate predictions vary because of the constraints on weights and because for different sets of fundamentals different time periods are considered depending on the availability of data. 
Table 6: 1-month ahead forecasts for the PPP, UIRP, monetary model and all fundamentals on the real-time data set: decoupled formulation.

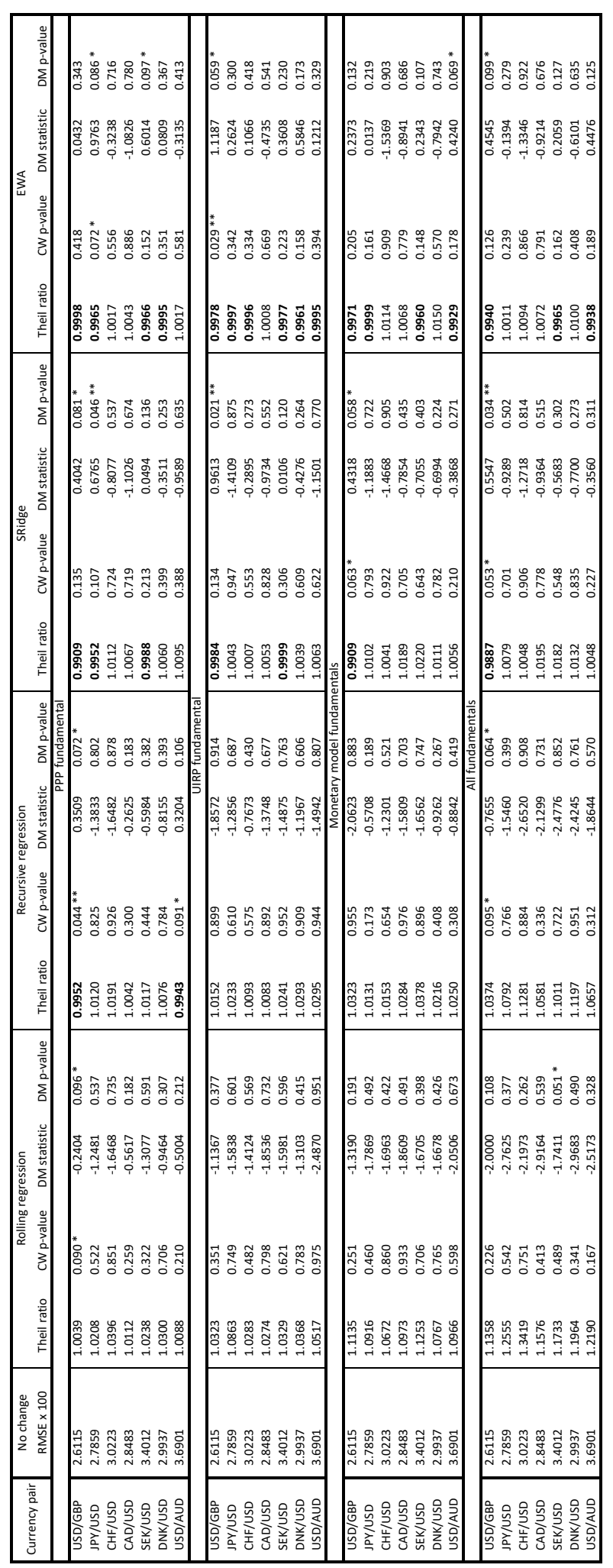

Notes: This table presents the results of forecasting end-of-month exchange rate 1-month ahead by the no-change prediction, the rolling OLS, recursive OLS, SRidge and EWA methods for decoupled fundamentals on real-time data. Column 1 shows the RMSE values for the no-change prediction. For each method, the Theil ratio (RMSE of the given method / RMSE of the no-change prediction), the CW-p-values, the DM statistic and its bootstrapped p-value are shown. The CW and DM tests are one-sided tests of equal out-of-sample prediction accuracy $\left(H_{0}\right)$ against superior out-of-sample prediction accuracy $\left(H_{1}\right)$ for the methods considered compared to the no-change prediction. ${ }^{* * *},{ }^{* *}$, and ${ }^{*}$ denote statistical significance at the $1 \%, 5 \%$, and $10 \%$ levels. Theil ratios $<1$ are indicated in bold. Sample period for the exchange rates: February 1999 - April 2017, data permitting (more details in Appendix A of the online supplementary material). 
Table 7: Economic criterion for evaluating forecasts on the real-time data set for the EWA algorithm based on decoupled fundamentals.

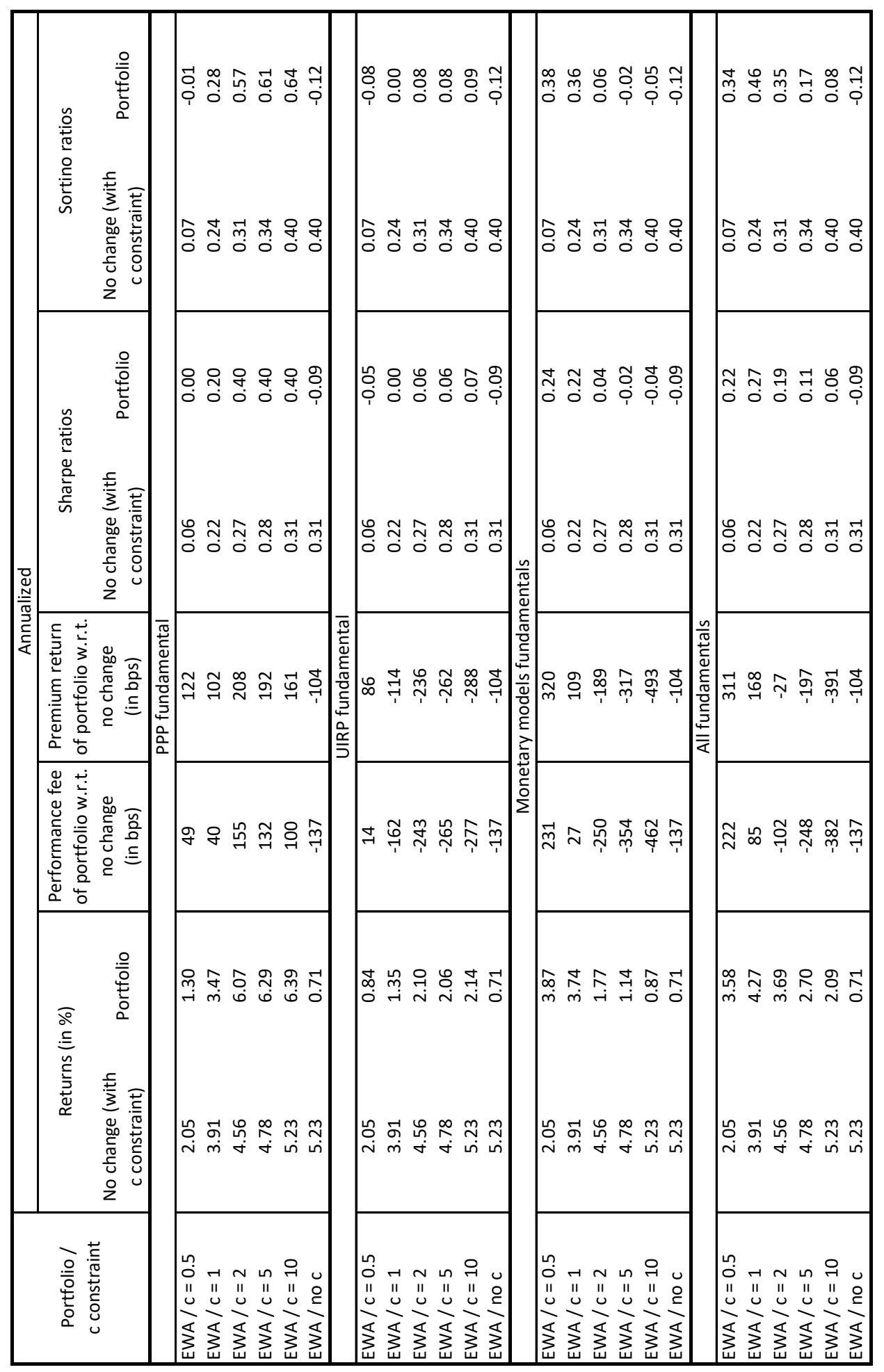

Notes: This table presents the comparison of performance of portfolios formed using forecasts from the EWA algorithm and the no-change prediction for the real-time data set based on decoupled fundamentals, using the procedure described in Section 5.4. Column 1 gives the conditions under which the portfolios were constructed. In columns 2 and 3 the annualized returns are given for the EWA and no-change prediction-based portfolios respectively. Column 4 shows the performance fee in basis points (bps) while column 5 the premium return (in bps) of the EWA-based portfolios relative to the ones formed based on the no-change forecast. The Sharpe and Sortino ratios for portfolios created using the two competing forecasting methods are reported in columns 6-10. For details on how these portfolios were created please consult Appendix C.3 of the online supplementary material. The results for the portfolios based on the no-change exchange rate predictions vary because of the constraints on weights and because for different sets of fundamentals different time periods are considered depending on the availability of data. 
Fundamentals and exchange rate forecastability with simple machine learning methods

\author{
Christophe Amat ${ }^{\mathrm{a}}$, Tomasz Michalski ${ }^{\mathrm{b}, *}$, Gilles Stoltz $^{\mathrm{c}}$ \\ ${ }^{a}$ Ecole Polytechnique, Palaiseau, France \\ ${ }^{b}$ HEC Paris - GREGHEC, Jouy-en-Josas, France \\ ${ }^{c}$ HEC Paris - CNRS, Jouy-en-Josas, France
}

\title{
Appendices published online
}

Posted at https://halshs.archives-ouvertes.fr/halshs-01003914
A. Detailed data description
B. Assessing forecast quality
C. Additional technical details (notes on computations and forecasts)
D. Additional graphs and tables 


\section{Appendix A. Detailed data description}

We recall that, as indicated in Section 4, the data used is freely available for download (Amat et al., 2018a).

The original fundamentals dataset of Molodtsova and Papell (2009) was extended to the period 1973-2014 whenever possible. For the discontinued time-series we used instead Datastream as the principal source. For those, as there were typically more time-series available without a clear advantage of one over the others, only those that were finally used to generate predictions presented in the paper are listed (we did not get qualitatively different results using the others). Data was obtained from Datastream on $30 / 01 / 2014$. When the code from the original data series was known, it was given instead. All series are monthly unless noted.

Quarterly time-series (there were only three of them) were transformed into monthly ones by local quadratic interpolation, i.e., a local quadratic polynomial was fit for each set of three adjacent quarterly observations; then, the monthly observations right before and after the central quarterly observation (the second one in the set) were imputed using the values of the quadratic polynomial. The output gap was estimated for each country with at least 24 data points. For the Hodrick-Prescott filter we used $\lambda=129600$ as advocated by Ravn and Uhlig (2002).

The exact data series (and the periods for which they could be used) are described in detail in Tables A.8-A.9 below. In particular, the three aforementioned quarterly time-series are indicated therein by a "quarterly" comment in their descriptions.

Real-time data on CPI, money stocks and industrial production for the period February 1999 to April 2017 were obtained from the OECD MEI data base (http://stats . oecd.org/mei/default.asp?rev=1) for countries whose currencies were not superseded by the euro (i.e., the British pound, Japanese yen, Swiss franc, Canadian dollar, Swedish krona, Danish kronor, Australian dollar and the comparison currency - the U.S. dollar). The time-series for M2 U.S. money supply until March 2001 (as there is a break in OECD reporting of M3/M2 series) were directly taken from the Federal Reserve historical releases website (https://www.federalreserve.gov/releases/h6/). 
Table A.8: Data series description, part I.

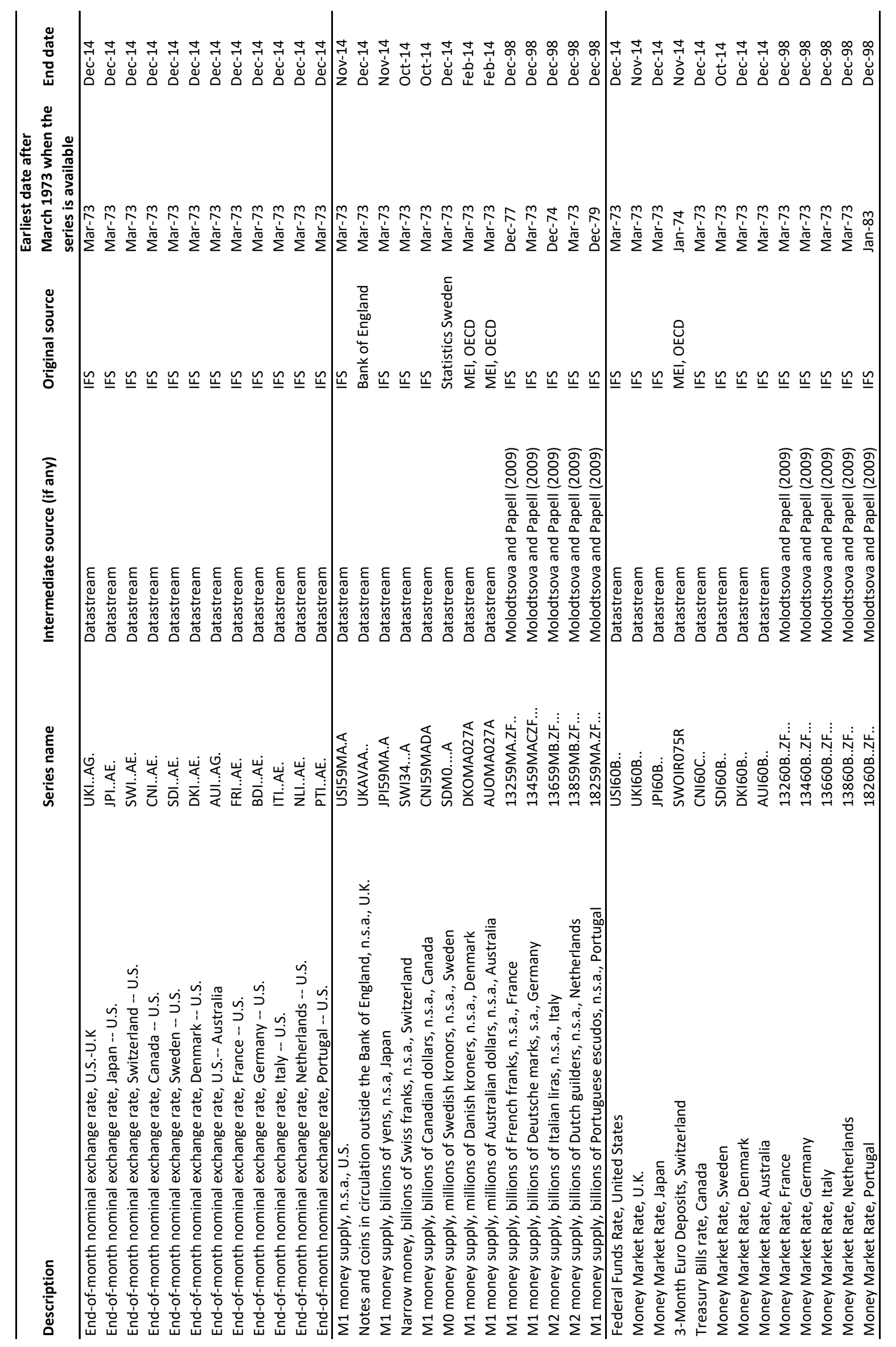


Table A.9: Data series description, part II.

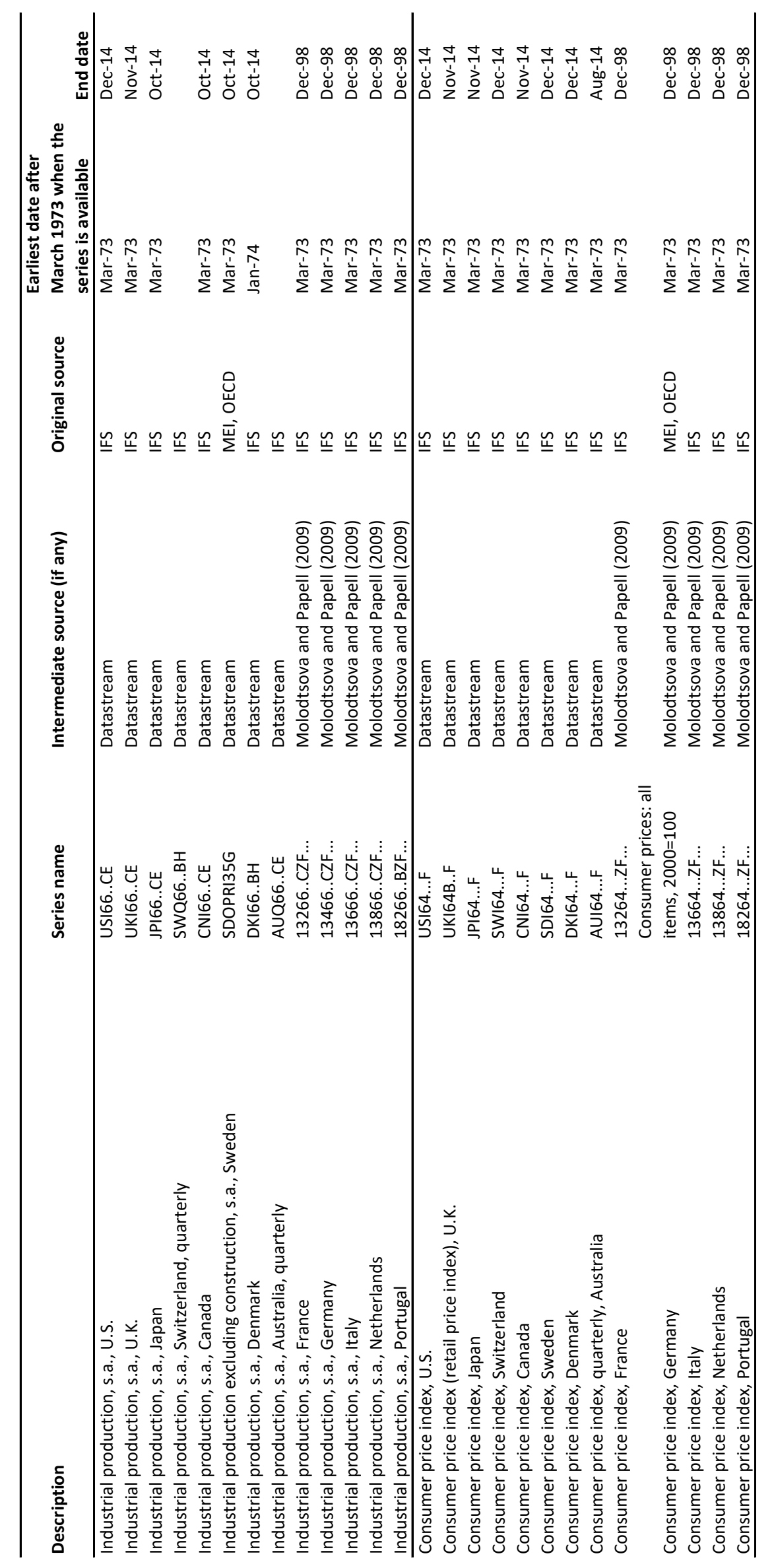




\section{Appendix B. Assessing forecast quality}

We denote by $T$ the total number of monthly values to be forecast, from months 1 to T. As is standard in the literature (see Molodtsova and Papell, 2009 and Rossi, 2013) and for essentially the same reasons, namely, the consideration of rolling OLS regressions, we allow for a training period of length $t_{0}=120$ months (10 years) and only evaluate the accuracy of the forecasts in months $t_{0}+1=121$ to $T$. To that end, we consider the root mean square error,

$$
\begin{aligned}
\text { RMSE } & =\sqrt{\frac{1}{T-t_{0}} \sum_{t=t_{0}+1}^{T}\left(\widehat{s}_{t}-s_{t}\right)^{2}} \\
& =\sqrt{\frac{1}{T-t_{0}} \sum_{t=t_{0}+1}^{T}\left(\left(\widehat{s}_{t}-s_{t-1}\right)-\left(s_{t}-s_{t-1}\right)\right)^{2}},
\end{aligned}
$$

and note that (by subtracting and adding the pivotal values $s_{t-1}$ ) this root mean square error is for both the logarithms of exchange rates $s_{t}$ and for changes in logarithms of exchange rates $s_{t}-s_{t-1}$.

We want to investigate whether the improvements in RMSE of one method over another are statistically significant. Denote by $\widehat{s}_{t}^{\prime}$ and $\widehat{s}_{t}$ the respective forecasts of two methods of interest. We aim to test the hypothesis $H_{0}$ that the difference in forecasting accuracy is not significant against the alternative hypothesis $H_{1}$ that the second method - the one outputting the forecasts $\widehat{s}_{t}$ - is significantly better, on average. To that end, the standard practice (see the two tests presented below) is to consider the instantaneous differences in accuracy

$$
d_{t}=\left(\widehat{s}_{t}-s_{t}\right)^{2}-\left(\widehat{s}_{t}-s_{t}\right)^{2} .
$$

We denote by

$$
\bar{d}_{T}=\frac{1}{T-t_{0}} \sum_{t=t_{0}+1}^{T} d_{t} \quad \text { and } \quad \bar{\sigma}_{T}^{2}=\frac{1}{T-t_{0}} \sum_{t=t_{0}+1}^{T}\left(d_{t}-\bar{d}_{T}\right)^{2}
$$

their empirical average and variance.

\section{Appendix B.1. Descriptive statistics}

In this section we consider the no-change prediction as the benchmark; it provides the forecasts $\widehat{s}_{t}^{\prime}=s_{t-1}$.

To get an initial feeling of whether $H_{0}$ should be rejected or not in this case, we can take a look at the quantiles of the sequence of $d_{t}$. For example, in Table D.14 of Appendix D, we see that in the sequential ridge regression with discount factors and the exponentially weighted average strategy with discount factors cases, the distribution of the differences $d_{t}$ is shifted toward positive values: the $75 \%$ and $90 \%$ quantiles are larger in absolute value than the $25 \%$ and $10 \%$ ones. This is not the case for rolling or recursive OLS regressions. Additional comments on these matters are provided at the end of Section 5.1. 


\section{Appendix B.2. A general test for comparing predictive accuracies}

In this section we sometimes still consider the no-change prediction as the benchmark: $\widehat{s}_{t}^{\prime}=s_{t-1}$, but also consider other benchmarks, like rolling or recursive OLS regression, e.g., in Table 3. We recall that we aim to test the hypothesis $H_{0}$ that the difference in forecasting accuracy between the method under scrutiny and the benchmark is not significant against the alternative hypothesis $H_{1}$ that the method - the one outputting the forecasts $\widehat{s}_{t}$ - is significantly better, on average.

The Diebold-Mariano test with relevant assumptions. Diebold and Mariano (1995) have presented a test relying directly on the differences $d_{t}$ in the forecasting errors, even though these may be serially correlated. Only mild and direct assumptions on the statistical behavior of these differences $d_{t}$ need to made. Here, we state their results with a rectangular lag, as they advocate.

More precisely, they showed that under assumptions of covariance stationarity and short memory of the differences $d_{t}$, for a properly chosen truncation lag denoted by $H \geqslant 0$, the test statistics

$$
S_{\mathrm{DM}, H}=\sqrt{T-t_{0}} \frac{\bar{d}_{T}}{\sqrt{\bar{\sigma}_{T}^{H, 2}}},
$$

where

$$
\bar{\sigma}_{T}^{H, 2}=\frac{1}{T-t_{0}} \sum_{t=t_{0}+1}^{T}\left(d_{t}-\bar{d}_{T}\right)^{2}+\frac{2}{T-t_{0}} \sum_{\tau=1}^{H} \sum_{t=\tau+t_{0}+1}^{T}\left(d_{t}-\bar{d}_{T}\right)\left(d_{t-\tau}-\bar{d}_{T}\right),
$$

converge to a $\mathcal{N}(0,1)$ distribution under $H_{0}$ and to $+\infty$ under $H_{1}$. Diebold (2012) emphasized the generality of the method to compare the predictive accuracy of forecasts between any two methods, as long as the mild assumption stated above, namely, covariance stationarity of the differences in accuracy $d_{t}$, holds. He explains in Section 2.2 of the mentioned reference why this assumption is natural and often met in practice.

The choice of the truncation lag $H$ was partially left open; Diebold and Mariano suggested choosing it as a function of the length of the short memory (of the autocovariance degree). Estimating by $\widehat{H}$ a proper $H$ based on our data, then substituting its value, would lead to considering the test statistic $S_{\mathrm{DM} . \widehat{H}}$, which would no longer be guaranteed to converge to a $\mathcal{N}(0,1)$ distribution under $H_{0}$. We instead take a more robust approach to reject $H_{0}$, that is, we build a more conservative test than the original test based on a good a priori value of $H$. Namely, we consider

$$
S_{\mathrm{DM}}=\sqrt{T-t_{0}} \frac{\bar{d}_{T}}{\sqrt{\max _{H \in\{0,1, \ldots, 20\}} \bar{\sigma}_{T}^{H, 2}}},
$$

which is smaller than any of the corresponding original statistics $S_{\mathrm{DM}, H}$. The limiting distribution under $H_{0}$ has a cumulative distribution function that is uniformly smaller than that of the $\mathcal{N}(0,1)$ distribution on positive numbers (while convergence to $+\infty$ is still achieved under $H_{1}$, at a slower rate). Note that for our data set a maximal value of 20 for $H$ was set because it corresponds roughly to the maximal value of $\sqrt{T-t_{0}}$ on our data. In the following, the test described above will be referred to as the DM test. 
We do not comment on the direct computation of the $p$-values for this DM test using quantiles of the normal distribution (which would be a conservative approach, i.e., the size and power of the test would be smaller than usual), as we rather resort to a bootstrapped approach to compute them.

Bootstrapped version. We follow here, as others have, the method and choices made by Mark and Sul (2001), Rogoff and Stavrakeva (2008), and Ince (2014). They all point out that the DM test is undersized and has less power than it should have when the conditions for its applications are not met, in particular, in the presence of forecast biases. They recommend computing its $p$-values based on a bootstrap procedure. Briefly, $M=$ 1000 bootstrapped simulations of the exchange rate and fundamentals time-series are computed, the relevant forecasting procedure (e.g., the exponentially weighted average strategy with discount factors) and its benchmark (e.g., the no-change predictor or rolling OLS regression) are applied to each simulation, and the resulting DM test statistic is computed (on each). This gives rise to an (empirical) distribution for the DM test statistic under $H_{0}$. Then, the DM test statistic is computed on the original data and the bootstrapped DM $p$-value is obtained, in a one-sided way, based on this distribution (it equals the empirical frequency of simulated DM test statistics that are above the original DM test statistic). These bootstrapped DM $p$-values are found in various tables of this article in the columns labeled "DM $p$-value" (without any specific reference to their bootstrap origin).

We now describe the method to simulate the time-series at hand. There are slight variations between the methods considered by Mark and Sul (2001), Rogoff and Stavrakeva (2008) and Ince (2014); we chose to stick to Rogoff and Stavrakeva (2008) here. For each country and each fundamental $j$, the data generating process is assumed to be

$$
\begin{aligned}
\Delta s_{t} & =\varepsilon_{t} \\
\Delta f_{j, t} & =\alpha_{j}+\beta_{j} t+\gamma_{j} f_{j, t-1}+\sum_{k=1}^{d} \delta_{j, k} \Delta s_{t-k}+\sum_{k=1}^{\ell} \zeta_{j, k} \Delta f_{j, t-k}+\varepsilon_{j, t}^{\prime},
\end{aligned}
$$

where $s_{t}$ and $f_{j, t}$ are defined as in Section 2.1, and $\Delta$ is shorthand for the unit variations

$$
\Delta s_{t}=s_{t}-s_{t-1} \quad \text { and } \quad \Delta f_{j, t}=f_{j, t}-f_{j, t-1},
$$

with $\varepsilon_{t}$ and $\varepsilon_{j, t}^{\prime}$ denoting the residual terms, which are modeled by random variables. That is, an autoregressive model on the evolution of the fundamentals is considered, with some external input given by the evolution of the exchange rate. For each country and each fundamental $j$, the parameters $d$ and $\ell$, as well as the coefficients $\delta_{j, k}$ and $\zeta_{j, k}$ of the autoregressive equation, are chosen separately. Given $d$ and $\ell$, the coefficients are estimated using OLS regression. The parameters $d$ and $\ell$ selected minimize Akaike's information criterion (AIC) over the choices considered, respectively, integers 1-10 for $d$ and 1-30 for $\ell$ (we set these intervals so that in our simulations, $d$ and $\ell$ rarely reach the upper values 10 and 30). These two parameters may differ across countries and fundamentals. AIC is also used to decide whether to include no constant and no trend, a constant and no trend, or a constant and a trend. Note in passing that the residuals $\varepsilon_{j, t}^{\prime}$ are also estimated by quantities denoted $\hat{\varepsilon}_{j, t}^{\prime}$. Once the data generating processes have been all estimated, the exchange rates $s_{t}$ and fundamentals $f_{j, t}$ are simulated recursively as follows. 
First, we need two inputs for this recursive simulation: (i) seeds to initiate the autoregressive simulations, and (ii) values for the stochastic residuals $\varepsilon_{j, t}^{\prime}$ and $\varepsilon_{t}$. The latter will be sampled with replacement among the $\hat{\varepsilon}_{j, \tau}^{\prime}$ and the $\Delta s_{\tau}$, where $\tau \in\{1, \ldots, T\}$. As for the seeds, they need to be in number $\max \{d, \ell\}$; we obtain them by sampling with replacement among the original vectors $\left(\Delta s_{\tau}, f_{1, \tau}, \ldots, f_{N, \tau}\right)$, where $\tau \in\{1, \ldots, T\}$. Note that we use here the case-by-case resampling also considered in Rogoff and Stavrakeva (2008); we do not consider blocks of vectors.

Last, we note that we actually simulate the process until round $T+100$ and discard the 100 first observations thus simulated, to obtain time-series of the same length $T$ as the original series. We do so, following Rogoff and Stavrakeva (2008), to minimize the influence of the choice of seeds and make sure that the re-simulated times series are in a steady state.

\section{Appendix B.3. A test for the case of nested forecasting equations?}

The tests by West (1996), Clark and West $(2006,2007)$ are designed for comparing models, while we are interested here only in comparing forecasts, and not in assuming the existence of models - see Diebold (2012) and Rossi (2013) for related discussions. Furthermore, the tests by Clark and West have been designed and studied for only the rolling and recursive OLS regression cases, while here we are obviously interested in alternative methods. This is underlined in the original papers and pointed out again by Rogoff and Stavrakeva (2008).

Nonetheless, for the sake of completeness, we computed $p$-values associated with the test developed by Clark and West for nested models (even though we consider "forecasting equations" only and not models). These $p$-values should merely be considered as statistical indicators of predictability. We believe that the most significant tests being performed in our study are the DM tests mentioned above. The readers will note that in our tables, the test by Clark and West is typically less conservative than the DM one (as seen in other empirical and theoretical studies).

Mathematical description of the test. For this test we restrict our attention to the nochange prediction as the benchmark method: $\widehat{s}_{t+1}^{\prime}=s_{t}$.

This no-change prediction is nested into the larger forecasting equation (1). In this case, assuming the existence of underlying models, Clark and West (2006) argued that the differences in forecasting abilities $d_{t}$ were an unfair measure, and advocated instead to use adjusted differences, which in our context would be

$$
a_{t}=d_{t}+\left(\widehat{s}_{t}-s_{t-1}\right)^{2},
$$

where $\widehat{s}_{t}$ is the prediction associated with the larger forecasting equation (1). We denote by

$$
\bar{a}_{T}=\frac{1}{T-t_{0}+1} \sum_{t=t_{0}+1}^{T} a_{t}
$$

the empirical average of the adjusted differences $a_{t}$ and

$$
\bar{S}_{T}^{2}=\frac{1}{T-t_{0}+1} \sum_{t=t_{0}+1}^{T}\left(a_{t}-\bar{a}_{T}\right)^{2}
$$


their empirical variance. The test statistic is equal to

$$
S_{\mathrm{CW}}=\sqrt{T-t_{0}+1} \frac{\bar{a}_{T}}{\sqrt{\bar{S}_{T}^{2}}} .
$$

The hypotheses tested are in terms of an underlying model (if we are ready to assume that one exists). Namely, $H_{0}^{\prime}$ : the true underlying model is the no-change model versus $H_{1}^{\prime}$ : the true underlying linear model uses at least one fundamental of (1). Here again, we see that the test concerns predictability, not forecastability. Under the assumption that residuals of the models form a martingale difference sequence, and provided that rolling and recursive OLS regressions are considered when selecting coefficients in (1), Clark and West (2006) showed that $S_{\mathrm{CW}}$ converges to a $\mathcal{N}(0,1)$ distribution under $H_{0}^{\prime}$ and to $+\infty$ under $H_{1}^{\prime}$. The $p$-values associated with the test thus constructed are found in the tables in the columns labeled "CW $p$-value"; they are based on quantiles of the normal distribution. In this article, this test will be referred to as the $\mathrm{CW}$ test.

We recall that as indicated several times above, these $p$-values are merely indicators, and of predictability rather than of forecastability. We include them here for the sake of completeness. 


\section{Appendix C. Additional technical details}

In this section, we provide

- explanations on how to run a single instance of EWA and SRidge in practice, by choosing their hyperparameters (Appendix C.1) well,

- some additional details on the numerical implementation of our machine-learning methods (Appendix C.2),

- a more complete description of the economic evaluation of our forecasting strategies, including a presentation of how to include constraints on the currency weights (Appendix C.3).

\section{Appendix C.1. EWA and SRidge in practice: how to choose their hyperparameters}

We recall that in our simulation study we do not report the performance of the EWA and SRidge methods for several well-chosen (hand-picked) sets of hyperparameters, as is usual in the literature. We instead re-use a more operational approach already considered in Devaine et al. (2013), based on a sequential grid search. We thus only report the results obtained for one run of the method. The aim of this section is to state all details of the grid-search approach followed.

For EWA, the single run selects at each time instance $t$, based on available data, the hyperparameters $\eta_{t}>0$ and $\gamma_{t}>0$ (rather than the fixed $\gamma$ initially considered) to be used in (8). We chose $\kappa=2$, which is common in the literature and allows us to have a theoretical bound given condition (10). More precisely, the parameters $\eta_{t}$ and $\gamma_{t}$ for the prediction of instance $t+1$ are respectively selected in the grids $\mathcal{E} / \sqrt{t}$ and $\mathcal{G}$, where

$$
\begin{aligned}
\mathcal{E} & =\left\{m \times 10^{k}, m \in\{1,2,5\} \text { and } k \in\{-4,-3,-2,-1\}\right\} \cup\{1\}, \\
\text { and } \quad \mathcal{G} & =\{0\} \cup\left\{m \times 10^{k}, \quad m \in\{1,2,5\} \text { and } k \in\{0,1,2\}\right\} \cup\{1000\} \\
& =\{0,1,2,5,10,20,50,100,200,500,1000\} .
\end{aligned}
$$

These grids are (somewhat) evenly spaced on a logarithmic scale. To predict instance $t+1$, we resort to (8), with the pair of parameters $(\eta, \gamma)$ in the grids $\mathcal{E}$ and $\mathcal{G}$, whose associated EWA strategy with sequence of learning parameters $\eta, \eta / \sqrt{1}, \ldots, \eta / \sqrt{t-1}$ and fixed discount factor $\gamma$ performed best overall on instances 1 to $t$.

We proceed similarly for SRidge: we take the same discount power $\kappa=2$ as in the EWA case and calibrate the hyperparameters $\lambda_{t}$ and $\gamma_{t}$ to be used for month $t+1$ based on past data, using the same grid $\mathcal{G}$ for the $\gamma$ parameters, and the grid for $\lambda$ given by

$$
\begin{aligned}
\Lambda & =\{0\} \cup\left\{m \times 10^{k}, \quad m \in\{1,2,5\} \text { and } k \in\{1,2,3\}\right\} \cup\{10000\} \\
& =\{0,1,2,5,10,20,50,100,200,500,1000,2000,5000,10000\} .
\end{aligned}
$$

\section{Appendix C.2. Notes on computations and forecasts}

Calculations were performed with the Scilab 5.5.0 software; the code is available upon request.

We recall that the training period for our methods was set at 120 months, which is standard in the literature, and that, correspondingly, the rolling OLS regressions were 
estimated with 120 months of past data at each instance. The computed coefficients of our forecasts vary greatly over time and it is difficult to discern any temporal patterns - also a feature known in the literature. This is not a surprise as our forecasting methods do not aim to estimate some model - they merely output efficient forecasts. The regularization and discount terms that are computed from past data are non-zero most of the time, and differ substantially between currencies, fundamentals used, and time periods. Most of the time the best discount factors are high - above 10 - which means that short-term trends are weighted most strongly.

We tried different grids from those in Appendix C.1. All grids were logarithmic, as recommended by machine learning theory. We found that a larger grid typically yielded small improvements in terms of RMSE with respect to the initial grid - but not always, as the RMSE of predictions can get worse. With a denser grid we may overfit the regularization and the discounting terms themselves. Also, smaller obtained RMSEs do not guarantee better DM tests being obtained; all depends on the errors of individual predictions. It appears that there is a large set of said parameters where the quality of predictions (measured by the RMSE) are qualitatively very similar, all corresponding to an improvement over the OLS methods.

\section{Appendix C.3. Economic evaluation of forecasting strategies}

We recall here the methodology introduced by Della Corte et al. $(2009,2011)$ and well-summarized in $\mathrm{Li}$ et al. (2015). We followed it scrupulously to produce our main economic evaluation results, namely, those reported in Table 5 in the "no $c$ " lines. We also considered two additional approaches that deal with known problems in economic criterion evaluation - by constraining the weights, which led to the lines with " $c=\ldots$ " in the main and certain other tables. These: Tables D.17 and D.18, show results under a summation-to-less-than-1 constraint for the weights. Below, we recall only the basic elements of the methodology for economic evaluation, so as to be able to describe the two modified approaches.

Brief description of the basic methodology for economic evaluation. We consider one domestic (US) bond, with risk-free return rate $r_{\text {free, } t}$, and $K$ foreign bonds, indexed by $k=1, \ldots, K$, with risk-free return rates in their local currencies (that would be used as UIRP fundamentals) but with risky returns $r_{k, t}$ in US dollars because (and only because) of the exchange-rate risk. The subscripts $t$ index the time instances, namely, months in our case. At each month $t-1$, an investor forecasts the evolution of the exchange rates using our methods, and based on these forecasts, picks weights $w_{t}=\left(w_{1, t}, \ldots, w_{K, t}\right)^{\mathrm{T}} \in \mathbb{R}^{K}$ determining how to invest their money in the foreign bonds for month $t$; these weights are real numbers. Their return in month $t$ equals

$$
\sum_{k=1}^{K} w_{k, t} r_{k, t}+\left(1-\sum_{k=1}^{K} w_{k, t}\right) r_{\text {free }, t}=r_{\text {free }, t}+\sum_{k=1}^{K} w_{k, t}\left(r_{k, t}-r_{\text {free }, t}\right) .
$$

Next, the returns $r_{k, t}$ are not yet known in month $t-1$, but their conditional means $\bar{r}_{k, t}$ and variance-covariance matrix $\Sigma_{t}$ can be assumed to be (where conditioning is with respect to the $r_{k, t-1}$ values for month $t-1$ ). Indeed, the covariance matrix $\Sigma_{t}$ used follows from an empirical estimation based on the past covariances, while the means $\bar{r}_{k, t}$ 
are given precisely by our forecasts. A target conditional volatility $\bar{\sigma}$ is then set and the weights are picked to optimize the following mean-variance problem:

$$
\begin{aligned}
\max _{w_{t} \in \mathbb{R}^{K}} & r_{\text {free }, t}+\sum_{k=1}^{K} w_{k, t}\left(\bar{r}_{k, t}-r_{\text {free }, t}\right), \\
\text { subject to } & w_{t}^{\mathrm{T}} \Sigma_{t} w_{t} \leqslant \bar{\sigma}^{2} .
\end{aligned}
$$

A closed-form solution exists when no further constraint is imposed on the weights.

Imposing further constraints on the weights. As indicated in the main text, the weights chosen as above often have large components - especially if the returns of some currencies are highly correlated - and are unstable over time. A good way to avoid such issues is to add a squared regularization term; given a parameter $c>0$, we want to perform the optimization

$$
\begin{aligned}
\max _{w_{t} \in \mathbb{R}^{K}} & r_{\text {free }, t}+\sum_{k=1}^{K} w_{k, t}\left(\bar{r}_{k, t}-r_{\text {free }, t}\right), \\
\text { subject to } & w_{t}^{\mathrm{T}} \Sigma_{t} w_{t} \leqslant \bar{\sigma}^{2} \\
\text { and } & \sum_{k=1}^{K} w_{k, t}^{2} \leqslant c .
\end{aligned}
$$

We provide no closed-form solution to this problem, and instead solve it numerically to obtain our results. As this constrained maximization problem is a particular case of what is called a quadratically constrained quadratic program (QCQP), its solution is equal to that of its Lagrangian dual problem, which is of the form: minimize a (nonlinear) function of finitely many variables $\lambda_{1}, \lambda_{2}, \ldots$, subject to positivity constraints on only the $\lambda_{i}$. See Boyd and Vandenberghe (2009, Section 5.2.4) for details on this equivalence. Now, the dual problem is easily solved numerically with the help of toolboxes from various software packages; in our case, we resorted to the optim routine of Scilab.

Further constraining the problem as

$$
\begin{aligned}
\max _{w_{t} \in \mathbb{R}^{K}} & r_{\text {free }, t}+\sum_{k=1}^{K} w_{k, t}\left(\bar{r}_{k, t}-r_{\text {free }, t}\right), \\
\text { subject to } & w_{t}^{\mathrm{T}} \Sigma_{t} w_{t} \leqslant \bar{\sigma}^{2} \\
\text { and } & \sum_{k=1}^{K} w_{k, t}^{2} \leqslant c \\
\text { and } & \sum_{k=1}^{K} w_{k, t} \leqslant 1,
\end{aligned}
$$

it remains a QCQP, so the methodology above still applies.

Details on these calculations (exact statements of the dual problems, code written to solve them, etc.) are available upon request. 


\section{Appendix D. Additional graphs and tables}

This section provides complementary results as well as tables omitted from the main text.

Tables with results discussed in the text.

- Tables D.10-D.13 give supplementary information to the basic results presented in Section 5 .

- Table D.14 gives the quantiles of the differences in forecasting error between the no-change prediction and the methods we look at.

- Table D.15 gives the results for a subsample spanning the whole range of observations from January 1980 until the end of our sample. Note that some results are not available for the PTE/USD pair as data on interest rates starts in 1983.

- Tables D.16-D.18 show robustness results for the economic evaluation of the prediction performance of the exponentially weighted average strategy with discount factors: with or without the Danish Krone, and with or without a summation-toless-than-1 constraint on the currency weights.

- Tables D.19-D.24 show our results for the shorter period discussed in Section 5.5, namely, February 1999-December 2014.

- Table D.25 shows the results for the Taylor-rule based exchange rate fundamentals in their "decoupled" (heterogenous) form.

Comments on the weights seen in the exponentially weighted average strategy with discount factors for forecasts with all fundamentals. By construction, the exponentially weighted average strategy with discount factors has a natural interpretation as being able to identify "scapegoat" fundamentals that could have been considered by traders in some periods as in the model of Bacchetta and van Wincoop (2004). This is because based on past data and discounting, the fundamentals that are best at predicting exchange rates in a given period are given more weight while forming predictions. It is insightful therefore to investigate the behavior of these weights with time. For the sake of brevity, we show the results in Figures D.1-D.3 only for the case of predictions with all fundamentals for JPY/USD, USD/GBP and CHF/USD. Note that these were the setups which did not necessarily perform well in comparison to parsimonious models such as those based solely on PPP or UIRP; however, they did much better overall than the OLS methods as measured by Theil ratios and in direct comparisons.

The codes for the various fundamentals are the following: PPP refers to inflation rates, UIRP to money-market interest rates; MONEY to money stocks, and $\mathrm{Y}$ to industrial production, while INTCH denotes changes in money market interest rates. Lastly, "nochange" refers to the no-change forecast that also is assigned a weight in the exponentially weighted average strategy with discount factors.

The conclusion from these figures is striking: typically only one fundamental is given a predominant weight in a given period, and most of the time for very short time intervals. In most cases, the fundamentals that are given the most weight pertain to money market 
interest rates and money supply, and for one country only. Rarely do we observe this behavior for inflation or industrial production. It would be consistent with the scapegoat model as well as the fickle nature of expectations about the fundamentals driving the exchange rate. This is, however, open to yet another interpretation. It also may point out that different shocks to different fundamentals (news) could be driving exchange rates at different times, and - given their prevalence in our data - monetary policy and financial market shocks are the most important. 
Table D.10: Relative forecasting performance of machine learning methods vs. rolling and recursive regressions based on coupled fundamentals.

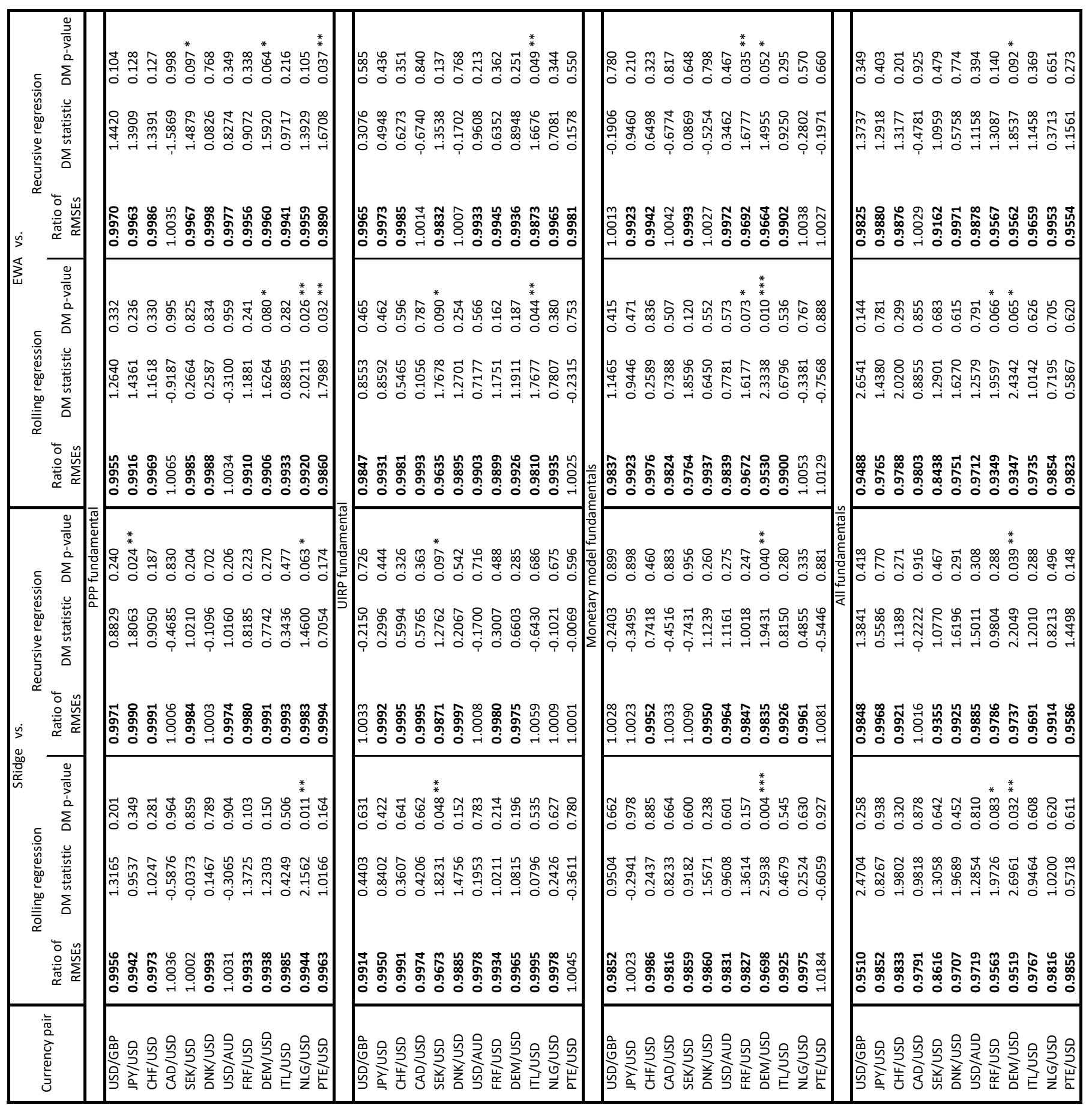

Notes: This table presents the comparison of forecasting performance between, respectively, SRidge and EWA vs. the rolling and recursive OLS for coupled fundamentals. Columns 1-6 show the comparison between SRidge and OLS methods, while columns 7-12 for EWA vs. OLS. For each comparison, the Theil ratio (RMSE of the given method / RMSE of the compared) and the DM statistic and its bootstrapped p-value are shown. The DM test is a one-sided test of equal out-of-sample prediction accuracy $\left(H_{0}\right)$ against superior out-of-sample prediction accuracy $\left(H_{1}\right)$ of the machine-learning method considered against the OLS methods. ***, **, and $*$ denote statistical significance at the $1 \%, 5 \%$, and $10 \%$ levels. Theil ratios $<1$ are indicated in bold. Sample period for the exchange rates: March 1973 - December 2014 unless shorter (indicated in Appendix A). 
Table D.11: Directional predictions of the exchange rates based on coupled fundamentals.

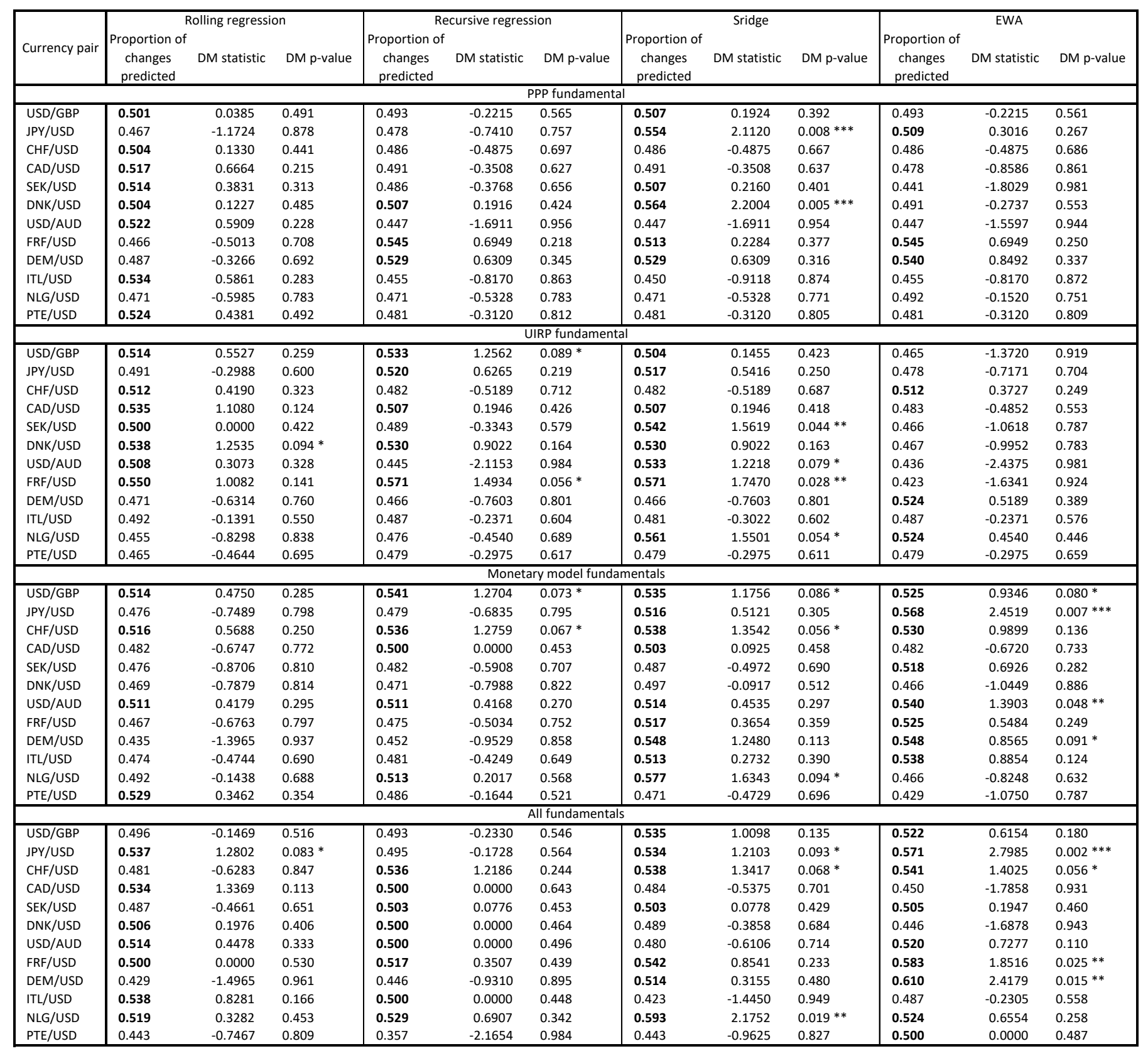

Notes: This table presents the results of forecasting the direction of change of the end-of-month exchange rate 1-month ahead by the rolling OLS, recursive OLS, SRidge and EWA methods for based on coupled fundamentals. For each method, the share of correctly forecasted changes, the DM statistic and its bootstrapped p-value are shown. The DM tests is a one-sided test of equal out-of-sample prediction accuracy $\left(H_{0}\right)$ against superior out-of-sample prediction accuracy $\left(H_{1}\right)$ for the methods considered against the benchmark of a $50 \%$ success rate. ${ }^{* * *},{ }^{* *}$, and ${ }^{*}$ denote statistical significance at the $1 \%, 5 \%$, and $10 \%$ levels. Shares $>0.5$ are indicated in bold. Sample period for the exchange rates: March 1973 - December 2014 unless shorter (indicated in Appendix A). 
Table D.12: Relative forecasting performance on the real-time data set of machine-learning methods vs. rolling and recursive regressions based on coupled fundamentals.

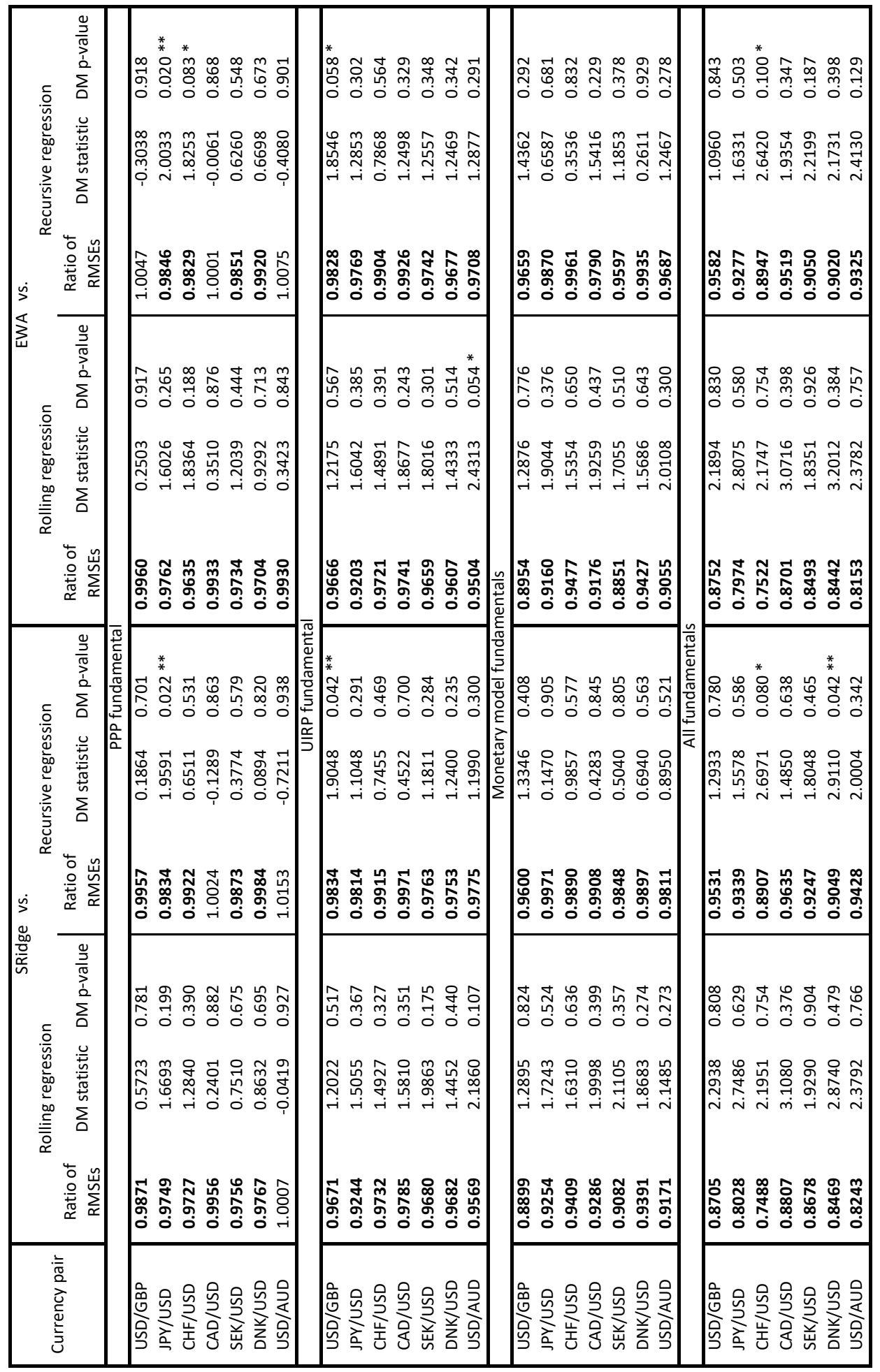

Notes: This table presents the comparison of forecasting performance between, respectively, SRidge and EWA vs. the rolling and recursive OLS for the real-time data set, based on decoupled fundamentals. Columns 1-6 show the comparison between SRidge and OLS methods, while columns 7-12 for EWA vs. OLS. For each comparison, the Theil ratio (RMSE of the given method / RMSE of the compared) and the DM statistic and its bootstrapped p-value are shown. The DM test is a one-sided test of equal out-of-sample prediction accuracy $\left(H_{0}\right)$ against superior out-of-sample prediction accuracy $\left(H_{1}\right)$ of the machine-learning method considered against the OLS methods. ${ }^{* *},{ }^{* *}$, and ${ }^{*}$ denote statistical significance at the 1\%, 5\%, and $10 \%$ levels. Theil ratios $<1$ are indicated in bold. Sample period for the exchange rates: February 1999 - December 2014. 


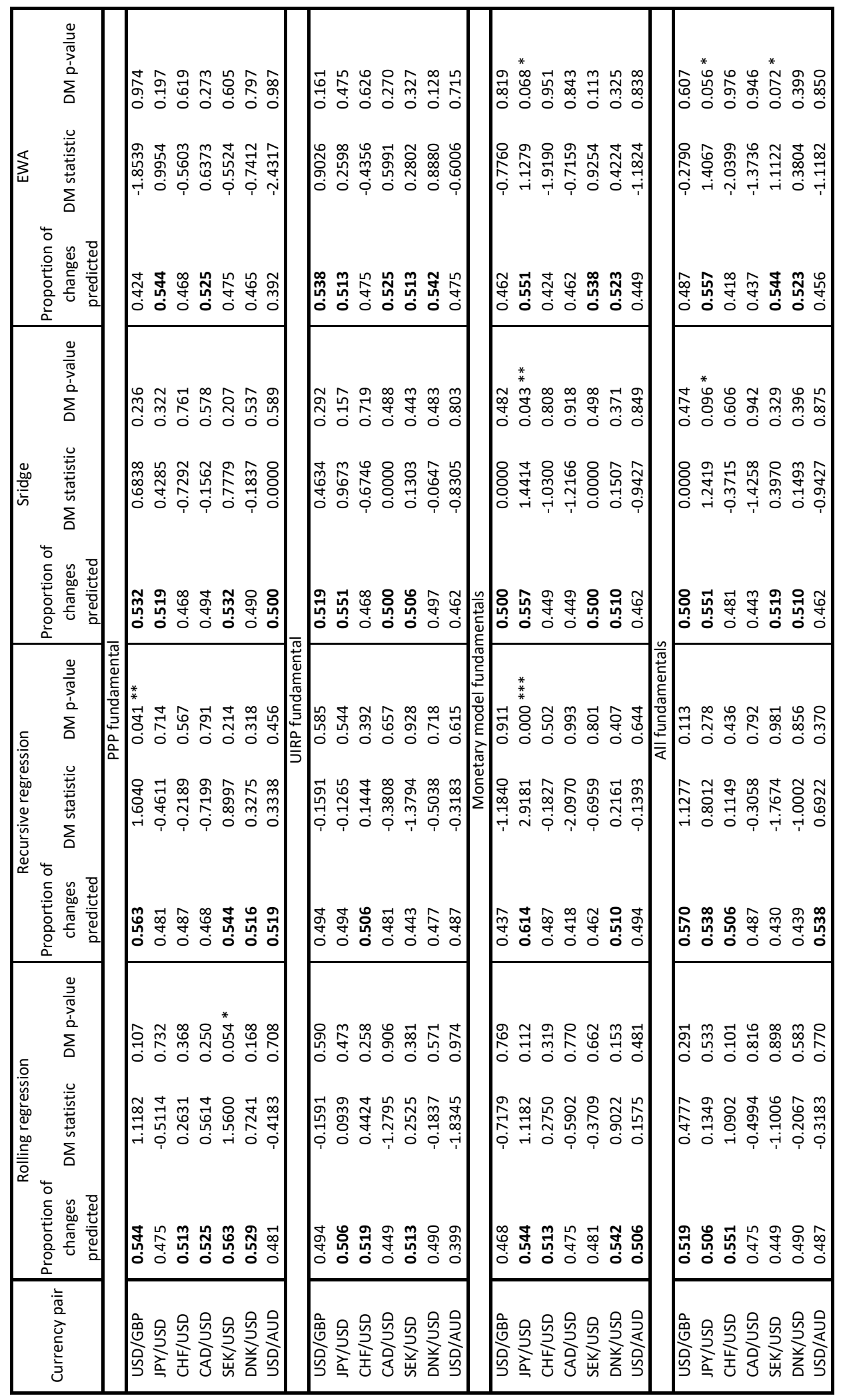

Notes: This table presents the results of forecasting the direction of change of the end-of-month exchange rate 1-month ahead by the rolling OLS, recursive OLS, SRidge and EWA methods for the real-time data set, based on decoupled fundamentals. For each method, the share of correctly forecasted changes, the DM statistic and its bootstrapped p-value are shown. The DM tests is a one-sided test of equal out-of-sample prediction accuracy $\left(H_{0}\right)$ against superior out-of-sample prediction accuracy $\left(H_{1}\right)$ of the methods considered against the benchmark of a $50 \%$ success rate. ${ }^{* * *},{ }^{* *}$, and ${ }^{*}$ denote statistical significance at the $1 \%, 5 \%$, and $10 \%$ levels. Shares $>0.5$ are indicated in bold. 
Table D.14: Distributions of the differences in forecasting error between the no-change prediction and the method under scrutiny based on decoupled fundamentals.

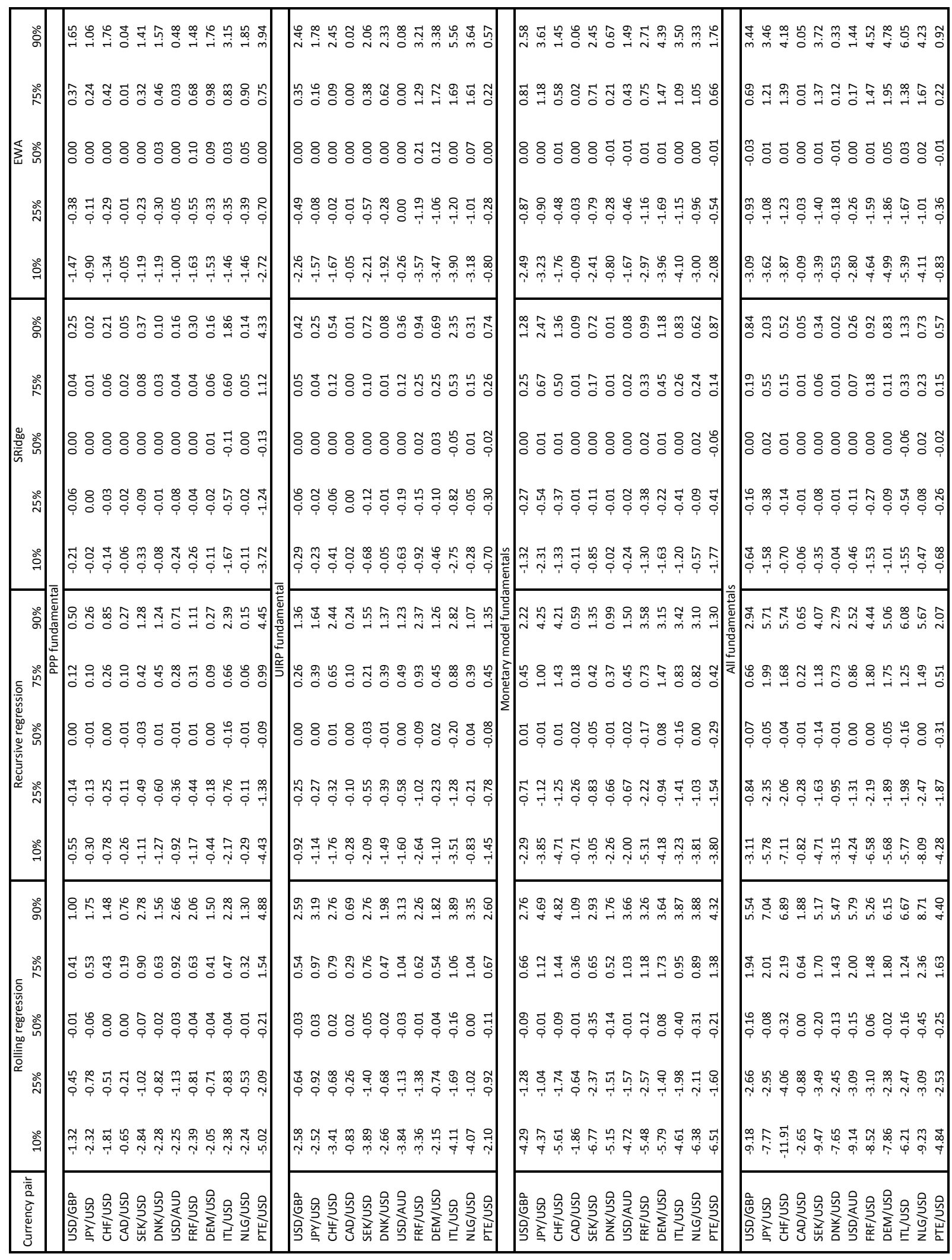

Notes: This table presents the results of forecasting end-of-month exchange rate 1-month ahead by the no-change prediction, the rolling OLS, recursive OLS, SRidge and EWA methods based on decoupled fundamentals. For each method, the time-series of the differences between the monthly forecasting errors of the no-change predictions and the ones of the method under scrutiny are computed. The table reports the quantiles of these time-series at the $10 \%, 25 \%, 50 \%, 75 \%$ and $90 \%$ levels, to give an idea of the distributions of the monthly forecasting errors. Distributions of errors that are shifted towards positive values indicate methods that outperfom the no-change prediction. Sample period for the exchange rates: March 1973 - December 2014 unless shorter (indicated in Appendix A). 
Table D.15: 1-month ahead forecasts for the PPP, UIRP, monetary model and all fundamentals: shorter-period sample (1980 onwards, decoupled fundamentals).

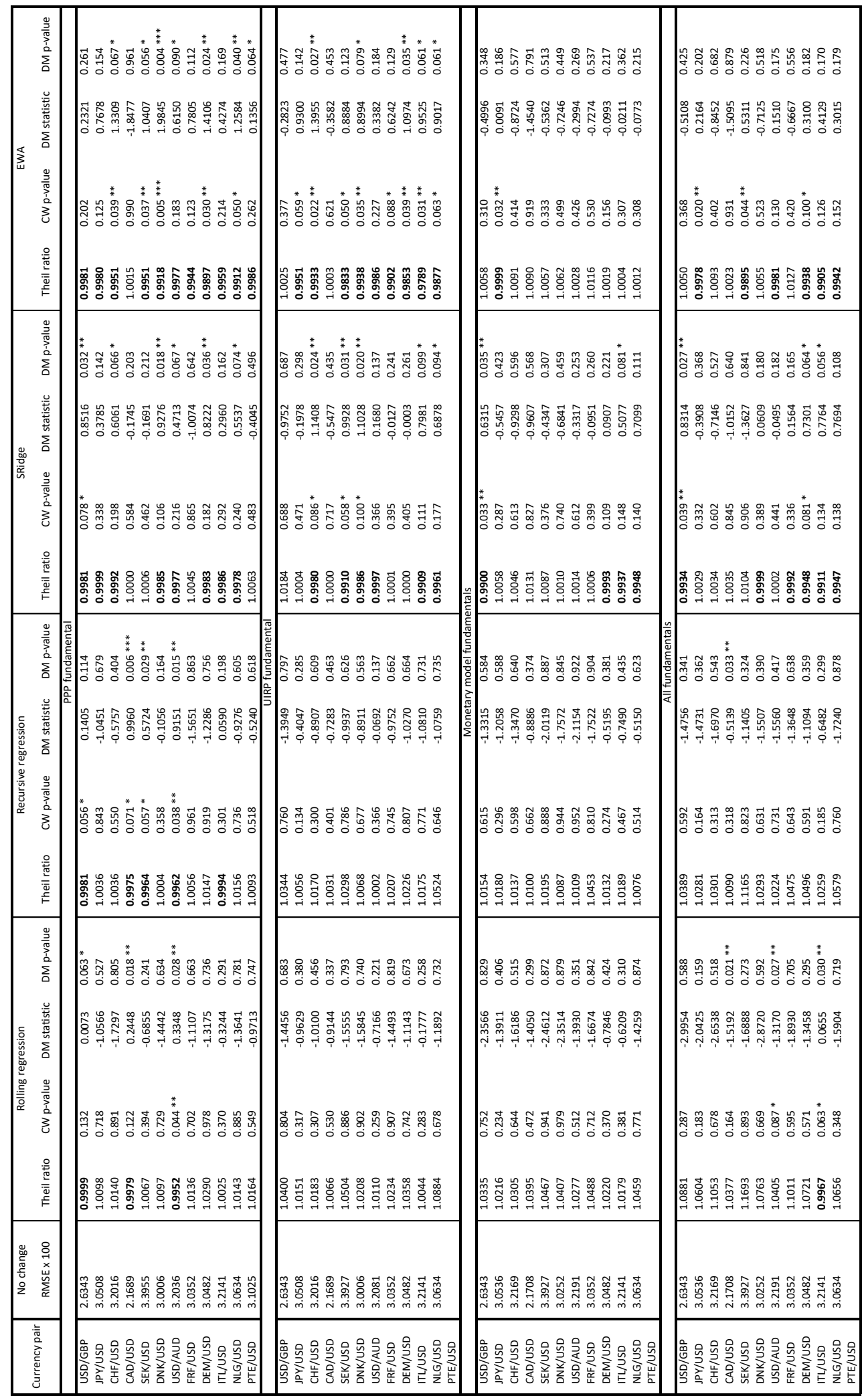

Notes: This table presents the results of forecasting end-of-month exchange rate 1-month ahead by the no-change prediction, the rolling OLS, recursive OLS, SRidge and EWA methods for decoupled fundamentals for the time period 1980-2014. Column 1 shows the RMSE values for the no-change prediction. For each method, the Theil ratio (RMSE of the given method / RMSE of the no-change prediction), the CW-p-values, the DM statistic and its bootstrapped p-value are shown. The CW and DM tests are one-sided tests of equal out-of-sample prediction accuracy $\left(H_{0}\right)$ against superior out-of-sample prediction accuracy $\left(H_{1}\right)$ for the methods considered compared to the no-change prediction. ${ }^{* * *}, * *$, and $*$ denote statistical significance at the $1 \%, 5 \%$, and $10 \%$ levels. Theil ratios $<1$ are indicated in bold. 
Table D.16: Economic criterion for evaluating forecasts for the EWA algorithm without the DNK/USD exchange rate.

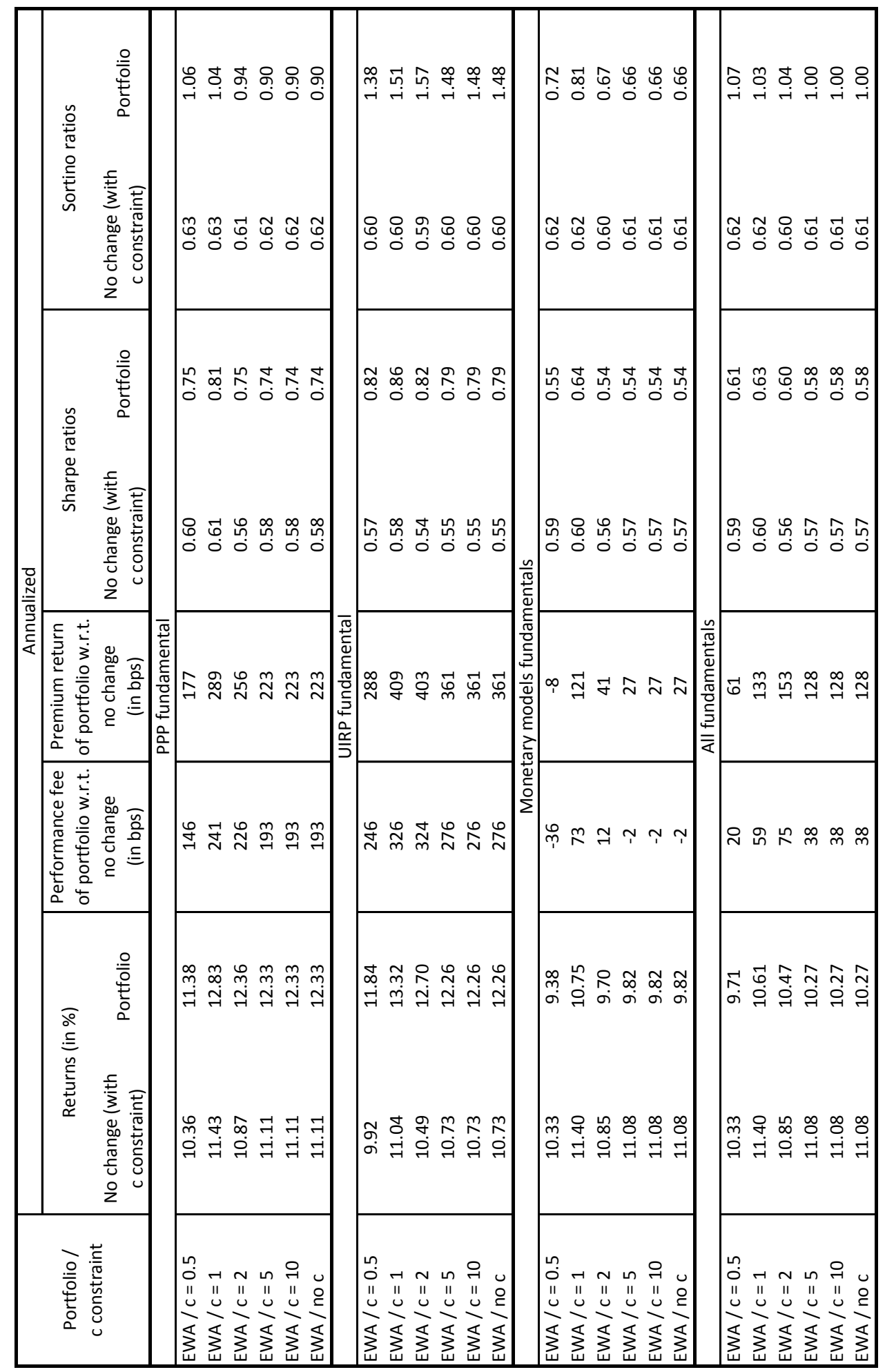

Notes: This table presents the comparison of performance of portfolios formed using forecasts from the EWA algorithm and the no-change prediction based on decoupled fundamentals without the DNK/USD exchange rate, using the procedure described in Section 5.4. Column 1 gives the conditions under which the portfolios were constructed. In columns 2 and 3 the annualized returns are given for the EWA and no-change prediction-based portfolios respectively. Column 4 shows the performance fee in basis points (bps) while column 5 the premium return (in bps) of the EWA-based portfolios relative to the ones formed based on the no-change forecast. The Sharpe and Sortino ratios for portfolios created using the two competing forecasting methods are reported in columns 6-10. For details on how these portfolios were created please consult Appendix C.3. The results for the portfolios based on the no-change exchange rate predictions vary because of the constraints on weights and because for different sets of fundamentals different time periods are considered depending on the availability of data. 
Table D.17: Economic criterion for evaluating forecasts for the EWA algorithm with an additional summation-to-less-than-1 constraint on the weights.

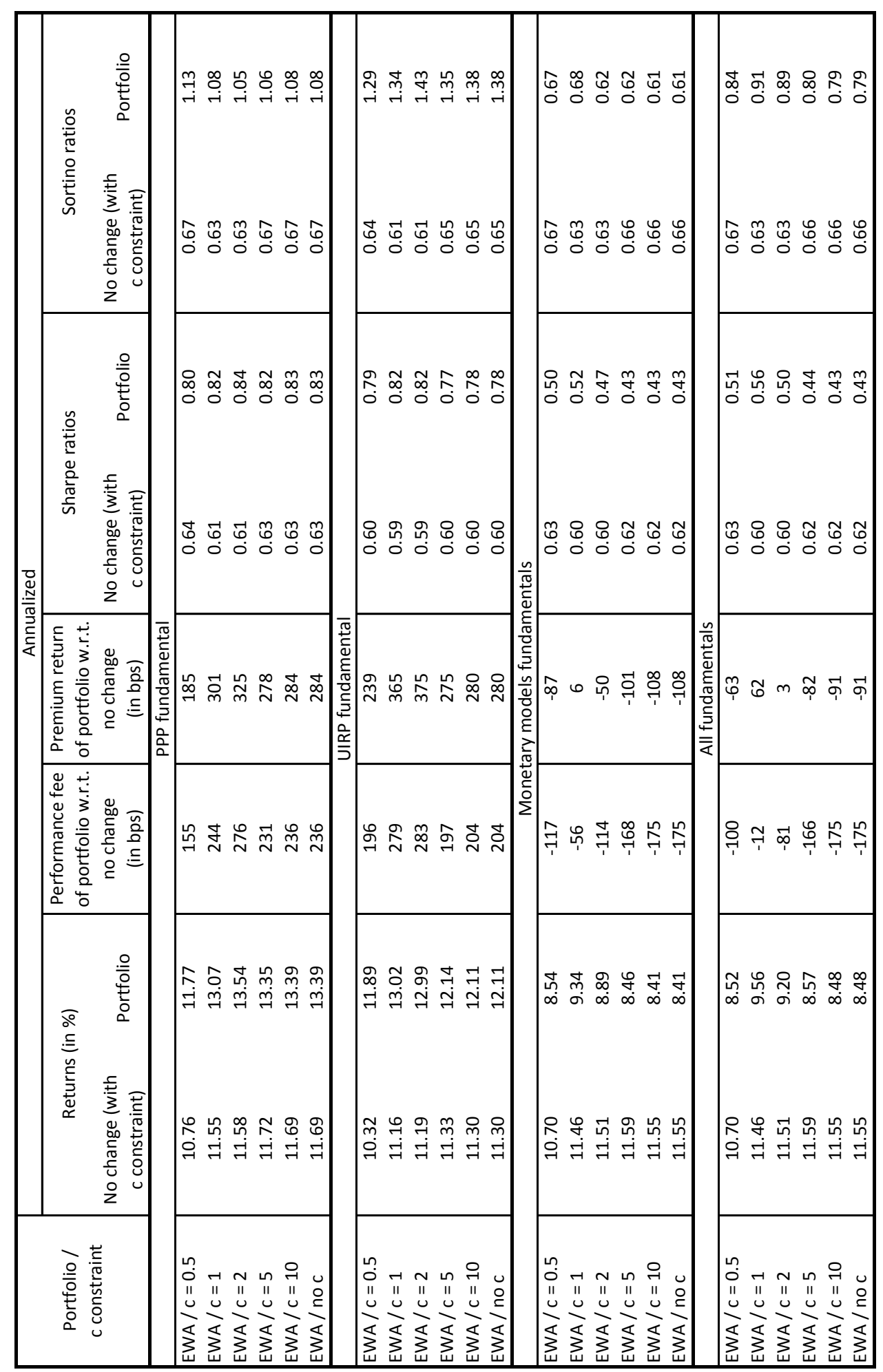

Notes: This table presents the comparison of performance of portfolios formed using forecasts from the EWA algorithm and the no-change prediction based on decoupled fundamentals with an additional summation-to-less-than- 1 constraint on the weights, using the procedure described in Section 5.4. Column 1 gives the conditions under which the portfolios were constructed. In columns 2 and 3 the annualized returns are given for the EWA and no-change prediction-based portfolios respectively. Column 4 shows the performance fee in basis points (bps) while column 5 the premium return (in bps) of the EWA-based portfolios relative to the ones formed based on the no-change forecast. The Sharpe and Sortino ratios for portfolios created using the two competing forecasting methods are reported in columns 6-10. For details on how these portfolios were created please consult Appendix C.3. The results for the portfolios based on the no-change exchange rate predictions vary because of the constraints on weights and because for different sets of fundamentals different time periods are considered depending on the availability of data. 
Table D.18: Economic criterion for evaluating forecasts for the EWA algorithm without the DNK/USD exchange rate but with an additional summation-to-less-than-1 constraint on the weights.

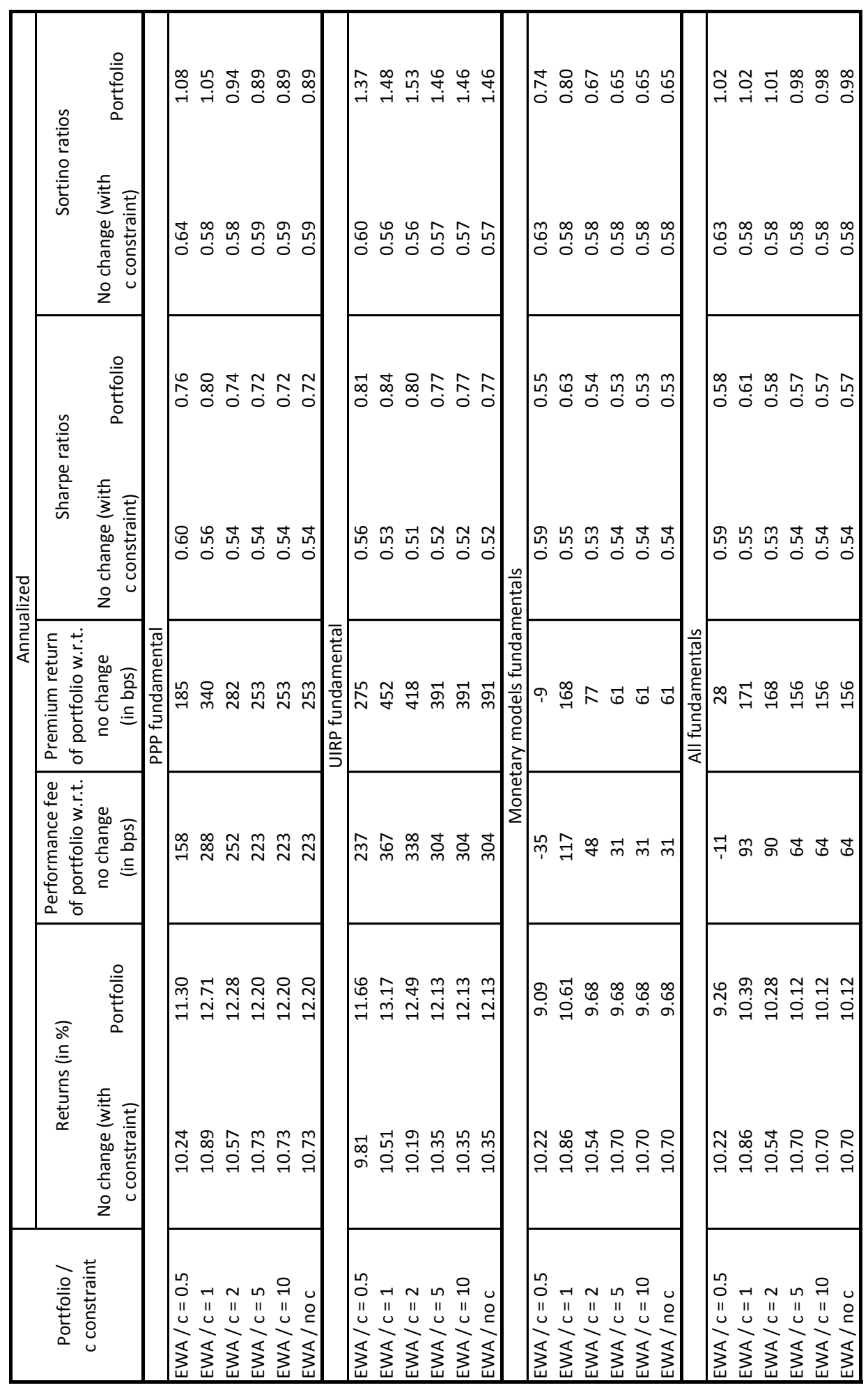

This table presents the comparison of performance of portfolios formed using forecasts from the EWA algorithm and the no-change prediction based on decoupled fundamentals with an additional summation-to-less-than-1 constraint on the weights and without the DNK/USD exchange rate, using the procedure described in Section 5.4. Column 1 gives the conditions under which the portfolios were constructed. In columns 2 and 3 the annualized returns are given for the EWA and no-change prediction-based portfolios respectively. Column 4 shows the performance fee in basis points (bps) while column 5 the premium return (in bps) of the EWA-based portfolios relative to the ones formed based on the no-change forecast. The Sharpe and Sortino ratios for portfolios created using the two competing forecasting methods are reported in columns 6-10. For details on how these portfolios were created please consult Appendix C.3. The results for the portfolios based on the no-change exchange rate predictions vary because of the constraints on weights and because for different sets of fundamentals different time periods are considered depending on the availability of data. 
Table D.19: 1-month ahead forecasts for the PPP, UIRP, monetary model and all fundamentals based on decoupled fundamentals, for a sample February 1999 - December 2014.

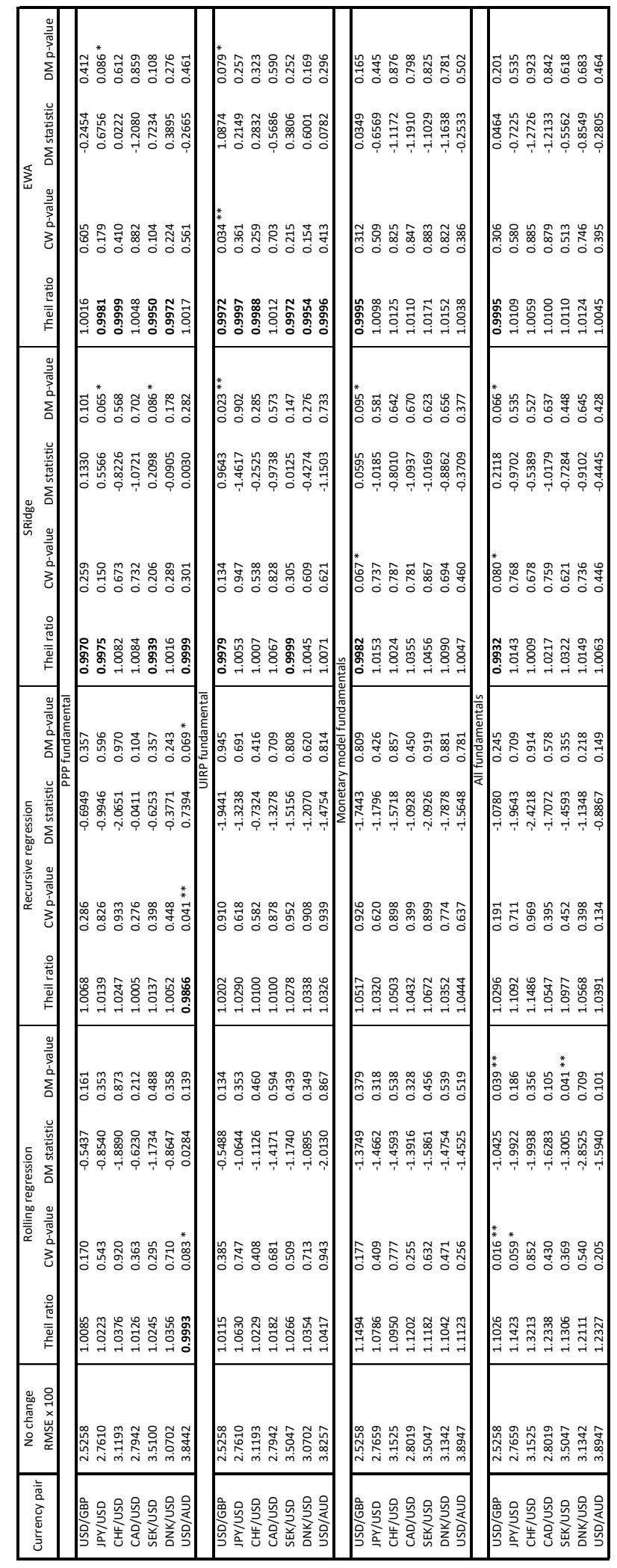

Notes: This table presents the results of forecasting end-of-month exchange rate 1-month ahead by the no-change prediction, the rolling OLS, recursive OLS, SRidge and EWA methods for decoupled fundamentals on a sample February 1999 - December 2014. Column 1 shows the RMSE values for the no-change prediction. For each method, the Theil ratio (RMSE of the given method / RMSE of the no-change prediction), the CW-p-values, the DM statistic and its bootstrapped p-value are shown. The CW and DM tests are one-sided tests of equal out-of-sample prediction accuracy $\left(H_{0}\right)$ against superior out-of-sample prediction accuracy $\left(H_{1}\right)$ for the methods considered compared to the no-change prediction. ${ }^{* *},{ }^{* *}$, and ${ }^{*}$ denote statistical significance at the $1 \%, 5 \%$, and $10 \%$ levels. Theil ratios $<1$ are indicated in bold. 
Table D.20: 1-month ahead forecasts on the real-time data set for the PPP, UIRP, monetary model and all fundamentals based on decoupled fundamentals, for a sample February 1999 - December 2014.

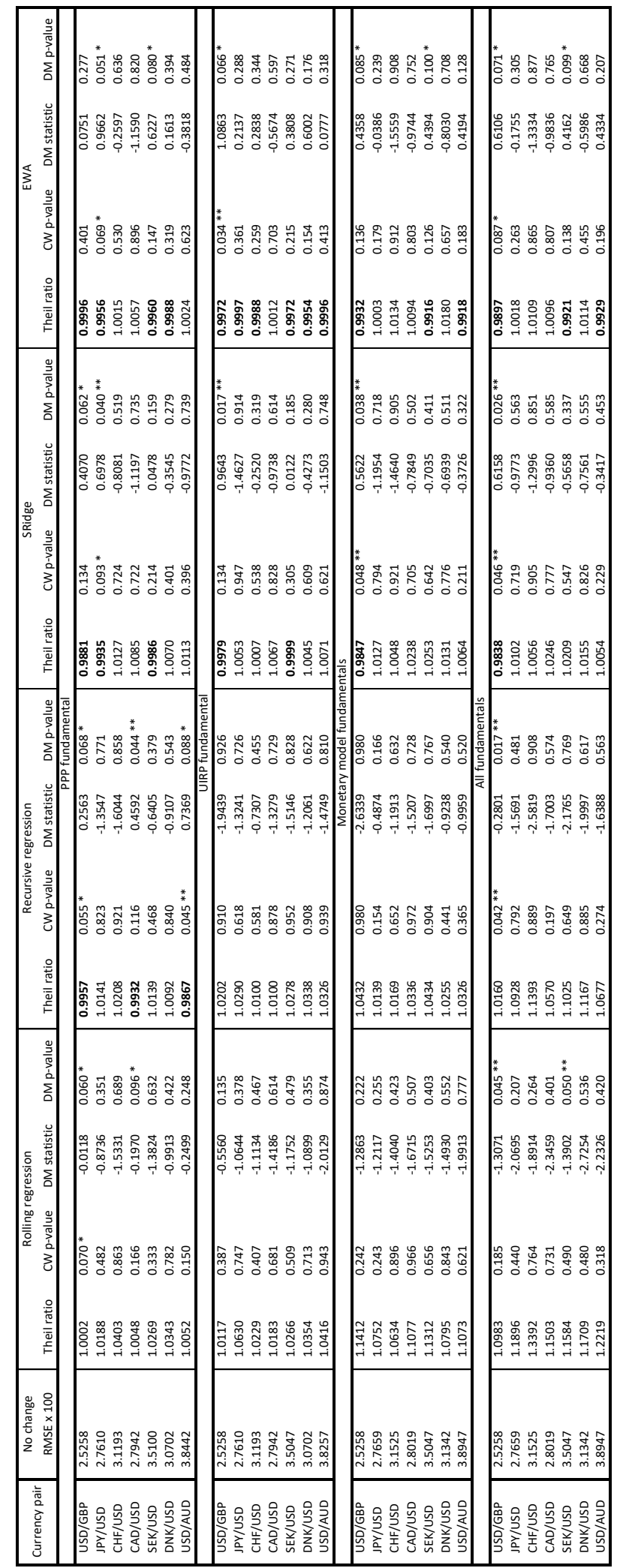

Notes: This table presents the results of forecasting end-of-month exchange rate 1-month ahead by the no-change prediction, the rolling OLS, recursive OLS, SRidge and EWA methods for decoupled fundamentals on a sample February 1999 - December 2014 using real time data. Column 1 shows the RMSE values for the no-change prediction. For each method, the Theil ratio (RMSE of the given method / RMSE of the no-change prediction), the CW-p-values, the DM statistic and its bootstrapped p-value are shown. The $\mathrm{CW}$ and DM tests are one-sided tests of equal out-of-sample prediction accuracy $\left(H_{0}\right)$ against superior out-of-sample prediction accuracy $\left(H_{1}\right)$ for the methods considered compared to the no-change prediction. ${ }^{* * *}$, **, and * denote statistical significance at the $1 \%, 5 \%$, and $10 \%$ levels. Theil ratios $<1$ are indicated in bold. 
Table D.21: Relative forecasting performance of the machine learning methods vs. rolling and recursive regressions based on decoupled fundamentals, for a sample February 1999 - December 2014.

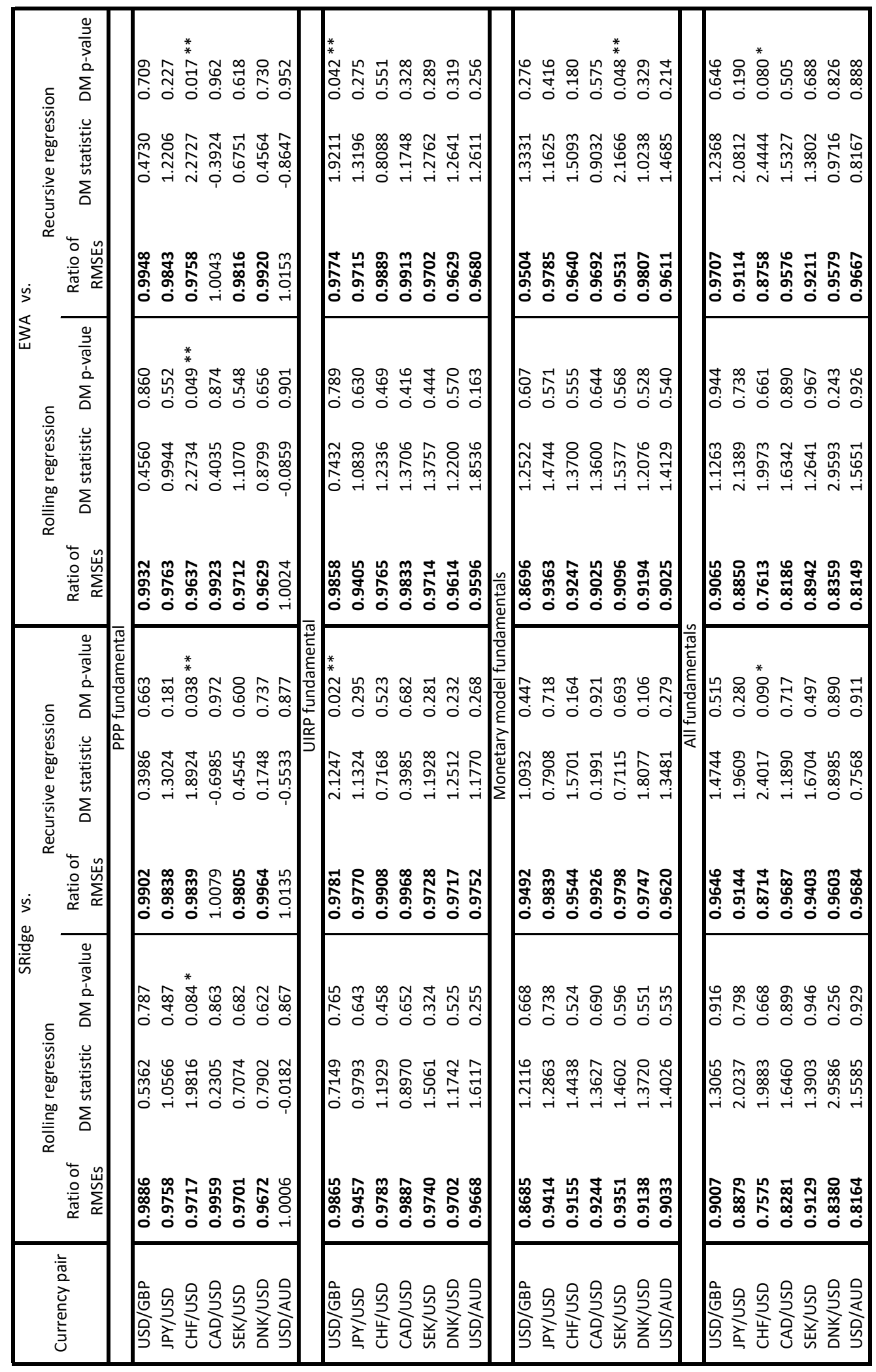

Notes: This table presents the comparison of forecasting performance between, respectively, SRidge and EWA vs. the rolling and recursive OLS for decoupled fundamentals for the period February 1999-December 2014. Columns 1-6 show the comparison between SRidge and OLS methods, while columns 7-12 for EWA vs. OLS. For each comparison, the Theil ratio (RMSE of the given method / RMSE of the compared) and the DM statistic and its bootstrapped p-value are shown. The DM test is a one-sided test of equal out-of-sample prediction accuracy $\left(H_{0}\right)$ against superior out-of-sample prediction accuracy $\left(H_{1}\right)$ of the machine-learning method considered against the OLS methods. ${ }^{* * *},{ }^{* *}$, and ${ }^{*}$ denote statistical significance at the $1 \%, 5 \%$, and $10 \%$ levels. Theil ratios $<1$ are indicated in bold. 
Table D.22: Relative forecasting performance on the real-time data set (February 1999 - December 2014) of the machine learning methods vs. rolling and recursive regressions based on decoupled fundamentals.

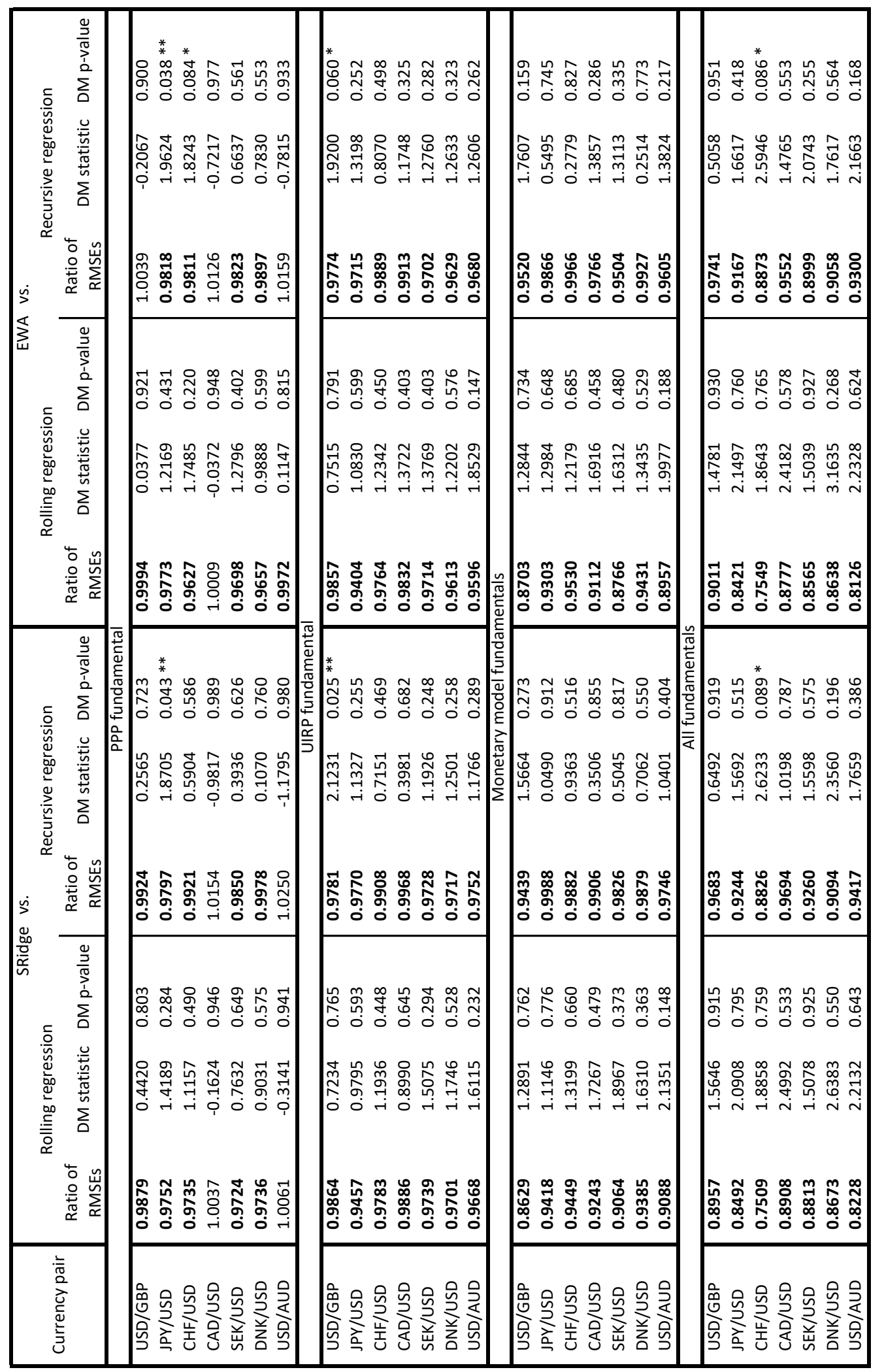

Notes: This table presents the comparison of forecasting performance between, respectively, SRidge and EWA vs. the rolling and recursive OLS for decoupled fundamentals for the period February 1999-December 2014 using real-time data. Columns 1-6 show the comparison between SRidge and OLS methods, while columns 7-12 for EWA vs. OLS. For each comparison, the Theil ratio (RMSE of the given method / RMSE of the compared) and the DM statistic and its bootstrapped p-value are shown. The DM test is a one-sided test of equal out-of-sample prediction accuracy $\left(H_{0}\right)$ against superior out-of-sample prediction accuracy $\left(H_{1}\right)$ of the machine-learning method considered against the OLS methods. $* * *, * *$, and ${ }^{*}$ denote statistical significance at the $1 \%, 5 \%$, and $10 \%$ levels. Theil ratios $<1$ are indicated in bold. 
Table D.23: Directional predictions of the exchange rates based on decoupled fundamentals, for a sample February 1999 - December 2014.

\begin{tabular}{|c|c|c|c|c|}
\hline 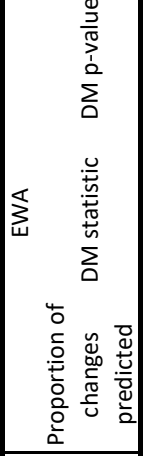 & 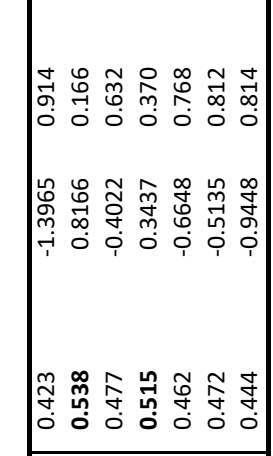 & 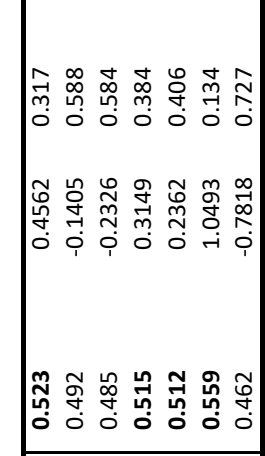 & 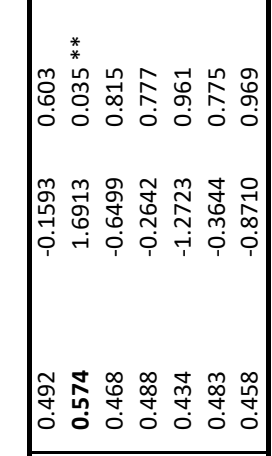 & 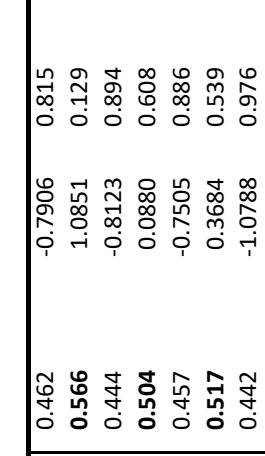 \\
\hline 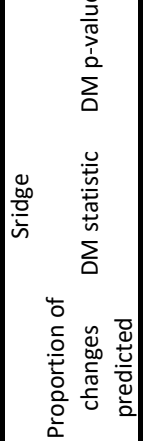 & 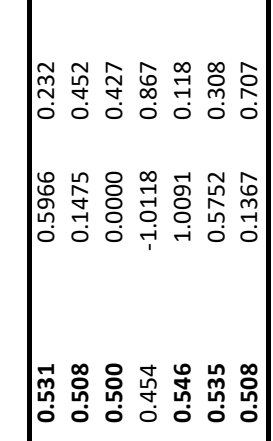 & 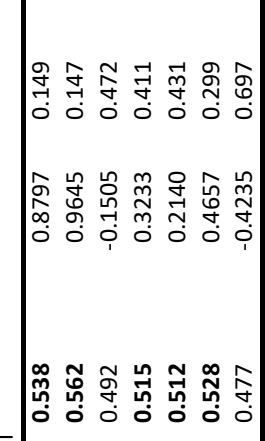 & 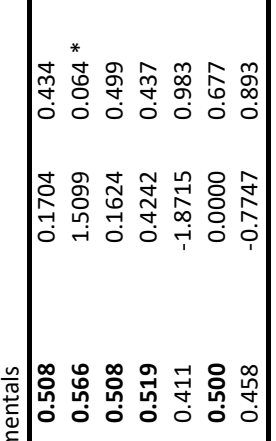 & 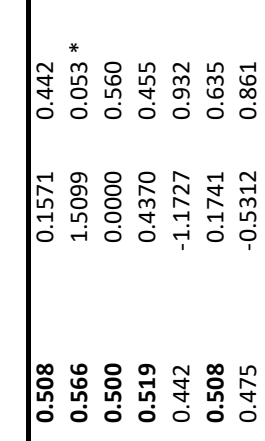 \\
\hline 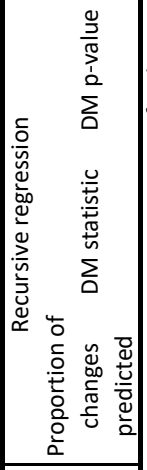 & 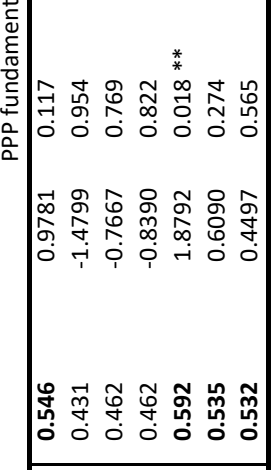 & 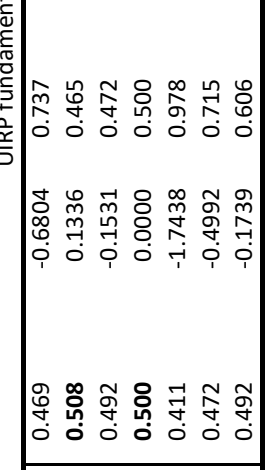 & 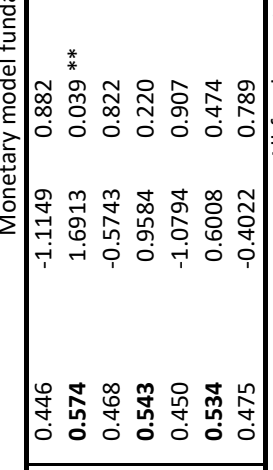 & 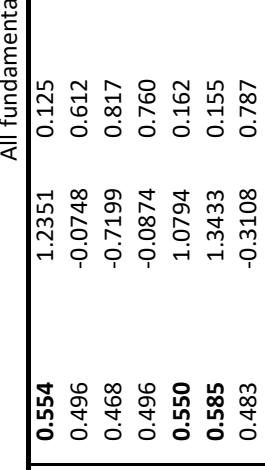 \\
\hline 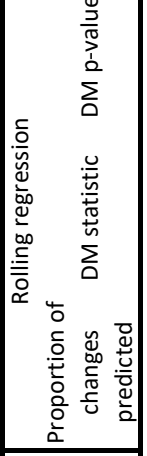 & 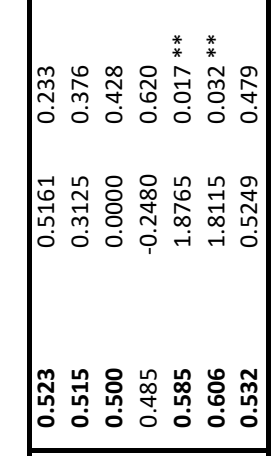 & 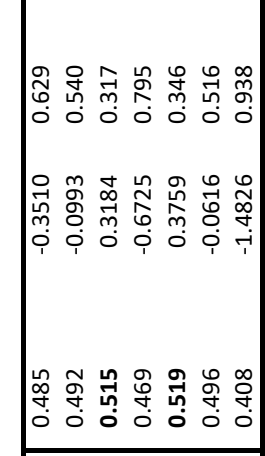 & 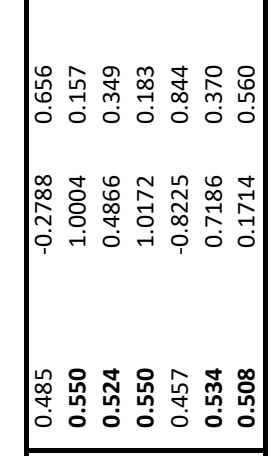 & 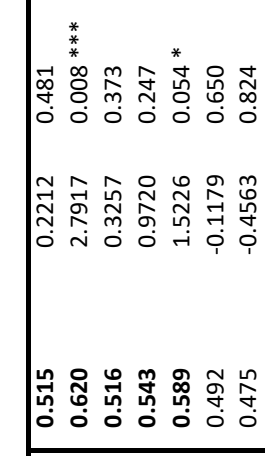 \\
\hline 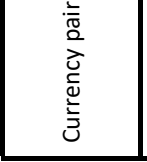 & 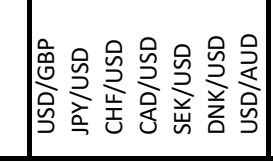 & 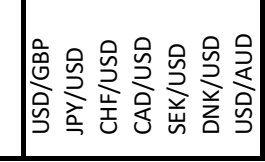 & 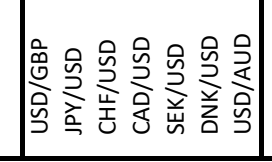 & 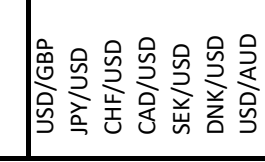 \\
\hline
\end{tabular}

Notes: This table presents the results of forecasting the direction of change of the end-of-month exchange rate 1-month ahead by the rolling OLS, recursive OLS, SRidge and EWA methods for a sample February 1999 - December 2014, based on decoupled fundamentals. For each method, the share of correctly forecasted changes, the DM statistic and its bootstrapped p-value are shown. The DM tests is a one-sided test of equal out-of-sample prediction accuracy $\left(H_{0}\right)$ against superior out-of-sample prediction accuracy $\left(H_{1}\right)$ for the methods considered against the benchmark of a $50 \%$ success rate. ${ }^{* * *},{ }^{* *}$, and ${ }^{*}$ denote statistical significance at the $1 \%, 5 \%$, and $10 \%$ levels. Shares $>0.5$ are indicated in bold. 
Table D.24: Directional predictions of the exchange rates on the real-time data set (February 1999 - December 2014) based on decoupled fundamentals.

\begin{tabular}{|c|c|c|c|c|}
\hline 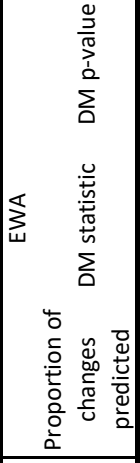 & 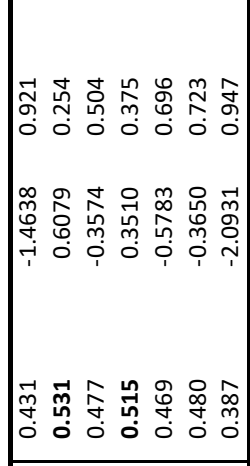 & 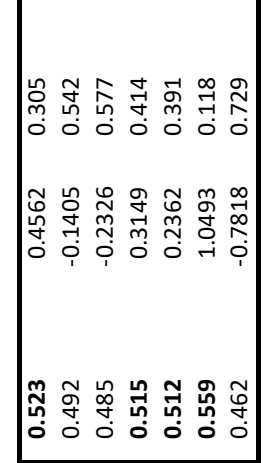 & 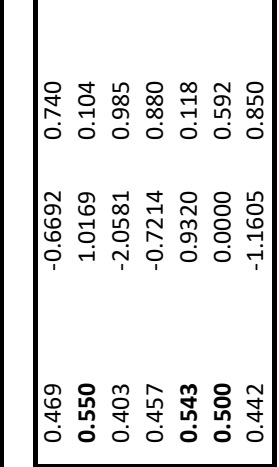 & 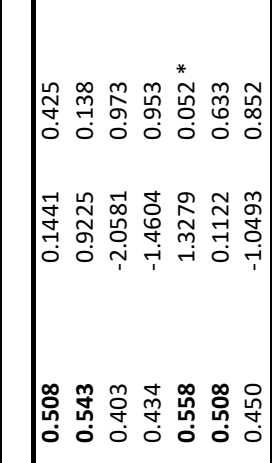 \\
\hline 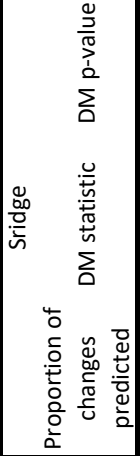 & 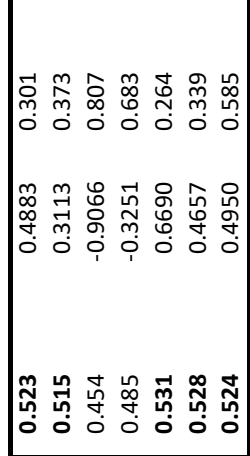 & 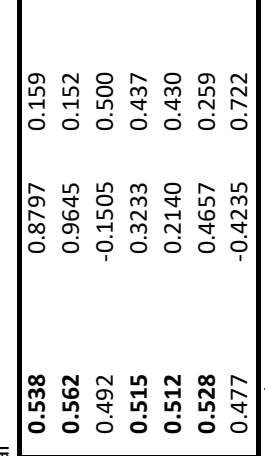 & 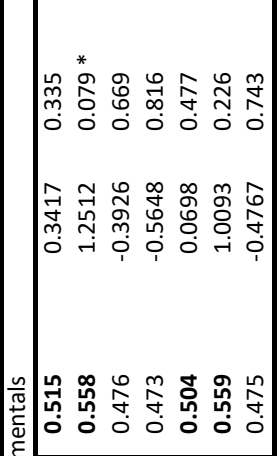 & 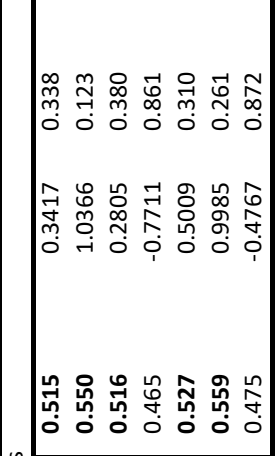 \\
\hline 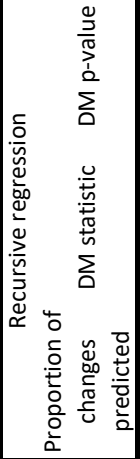 & 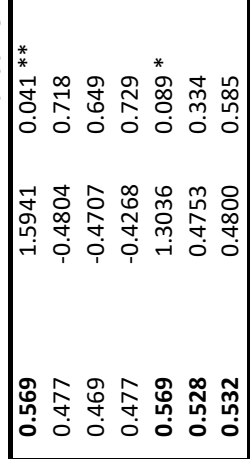 & 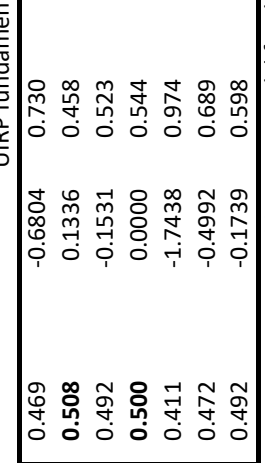 & 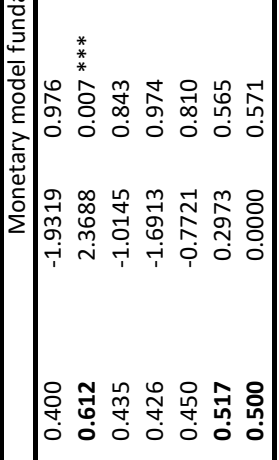 & 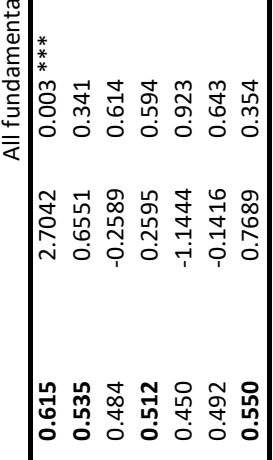 \\
\hline 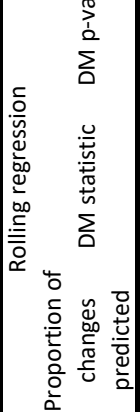 & 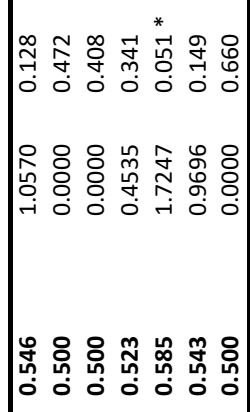 & 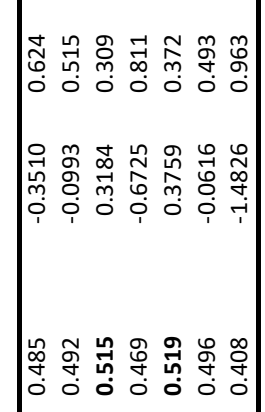 & 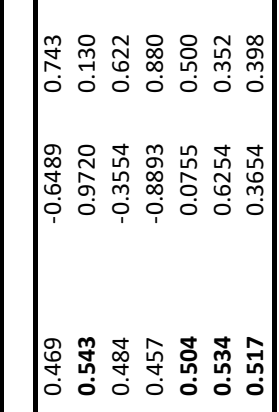 & 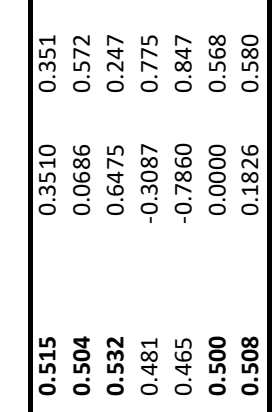 \\
\hline 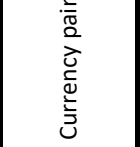 & 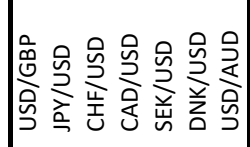 & 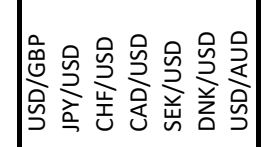 & 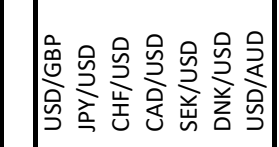 & 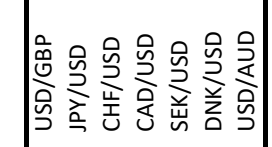 \\
\hline
\end{tabular}

Notes: This table presents the results of forecasting the direction of change of the end-of-month exchange rate 1-month ahead by the rolling OLS, recursive OLS, SRidge and EWA methods based on decoupled fundamentals for the period February 1999-December 2014 using real-time data. For each method, the share of correctly forecasted changes, the DM statistic and its bootstrapped p-value are shown. The DM tests is a one-sided test of equal out-of-sample prediction accuracy $\left(H_{0}\right)$ against superior out-of-sample prediction accuracy $\left(H_{1}\right)$ for the methods considered against the benchmark of a $50 \%$ success rate. ${ }^{* * *},{ }^{* *}$, and ${ }^{*}$ denote statistical significance at the $1 \%, 5 \%$, and $10 \%$ levels. Shares $>0.5$ are indicated in bold. 
Table D.25: 1-month ahead forecasts using decoupled Taylor-rule fundamentals.

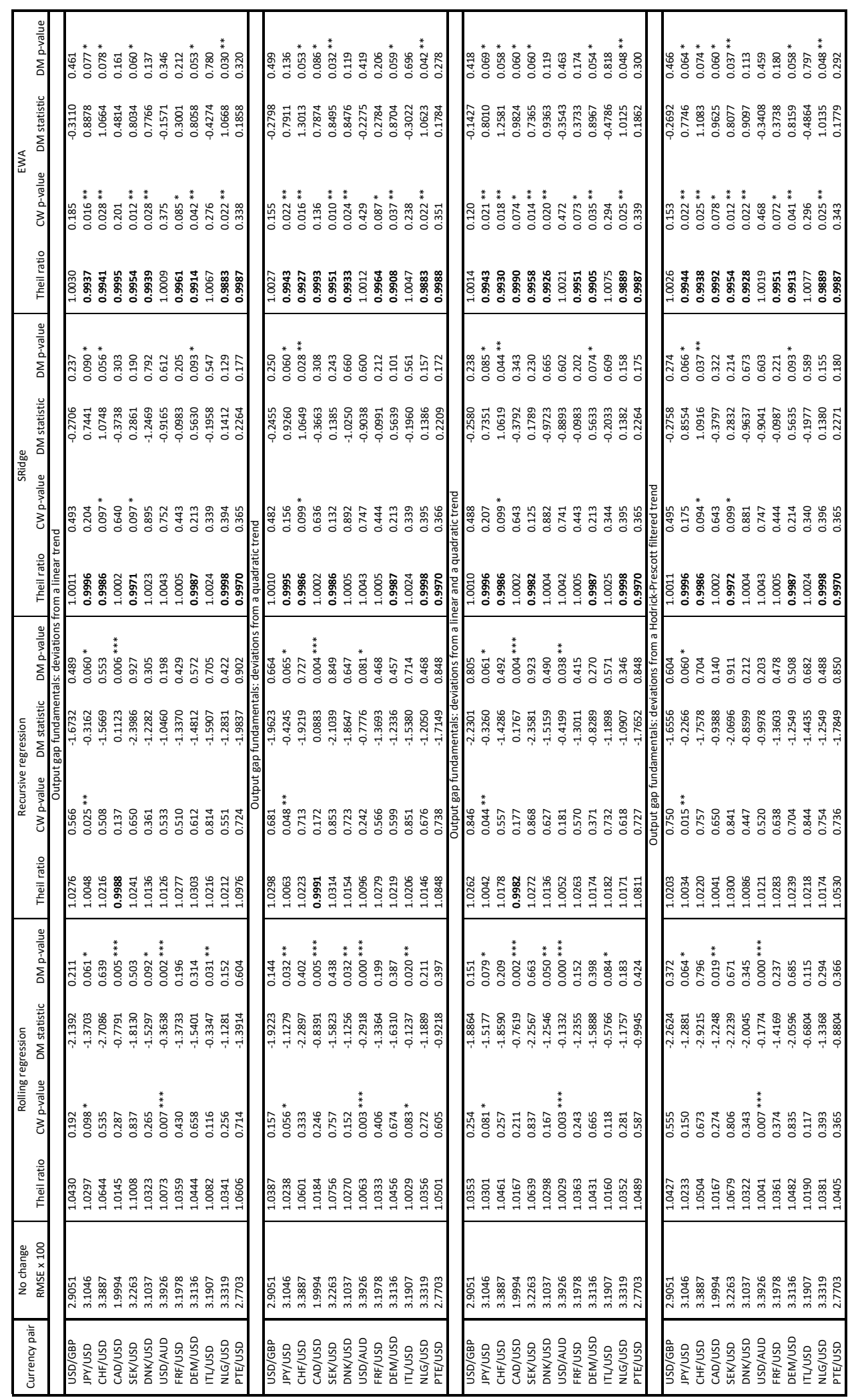

Notes: This table presents the results of forecasting end-of-month exchange rate 1-month ahead by the no-change prediction, the rolling OLS, recursive OLS, SRidge and EWA methods for different sets of Taylor-rule fundamentals. Column 1 shows the RMSE values for the no-change prediction. For each method, the Theil ratio (RMSE of the given method / RMSE of the no-change prediction), the CW-p-values, the DM statistic and its bootstrapped p-value are shown. The CW and DM tests are one-sided tests of equal out-of-sample prediction accuracy $\left(H_{0}\right)$ against superior out-of-sample prediction accuracy $\left(H_{1}\right)$ of the methods considered compared to the no-change prediction. ${ }^{* * *},{ }^{* *}$, and ${ }^{*}$ denote statistical significance at the $1 \%, 5 \%$, and $10 \%$ levels. Theil ratios $<1$ are indicated in bold. Sample period for the exchange rates: March 1973 - December 2014 unless shorter (indicated in Appendix A). 


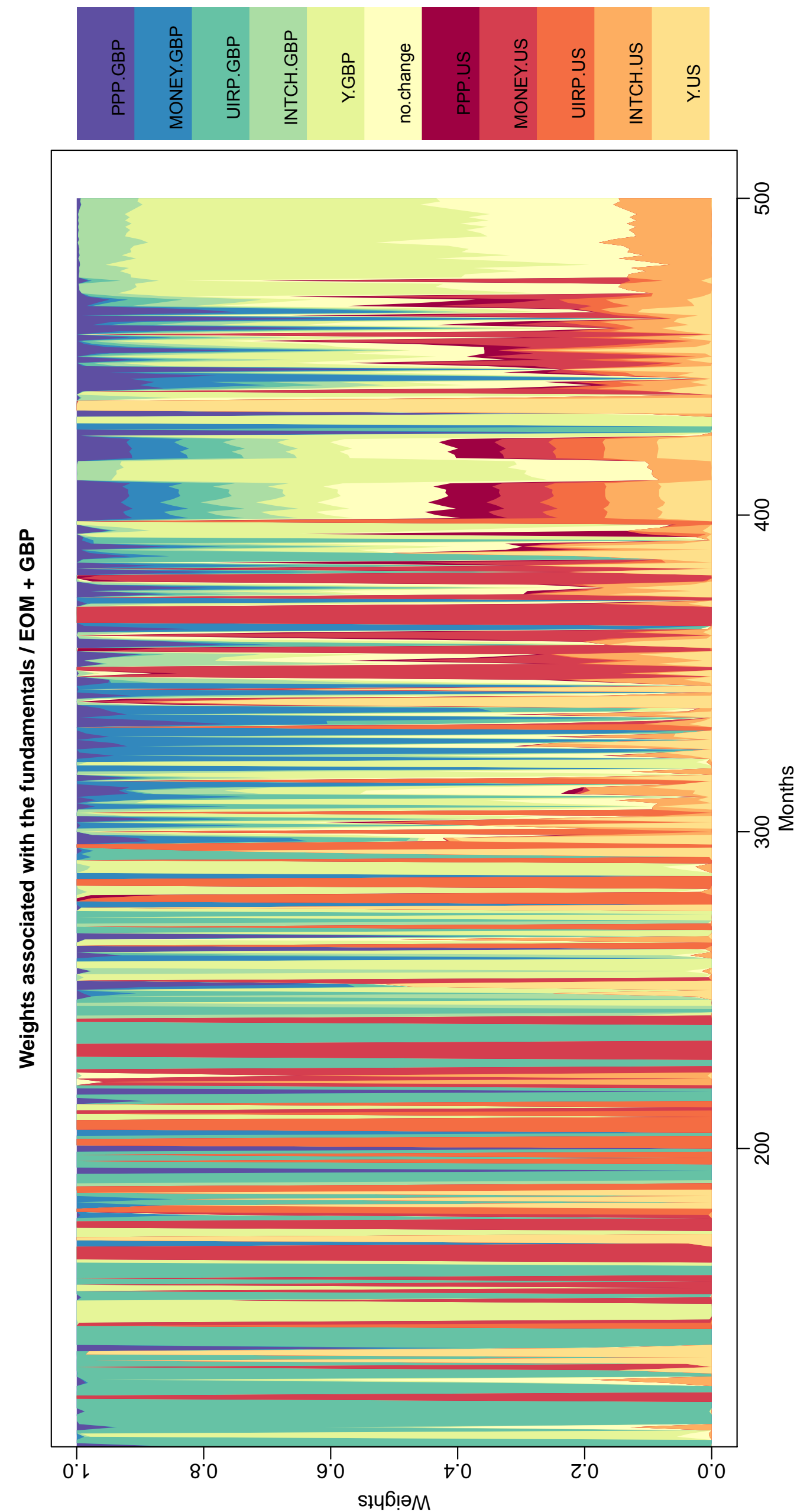

Figure D.1: Weights of different fundamentals in prediction: the USD/GBP case, all fundamentals considered together. 


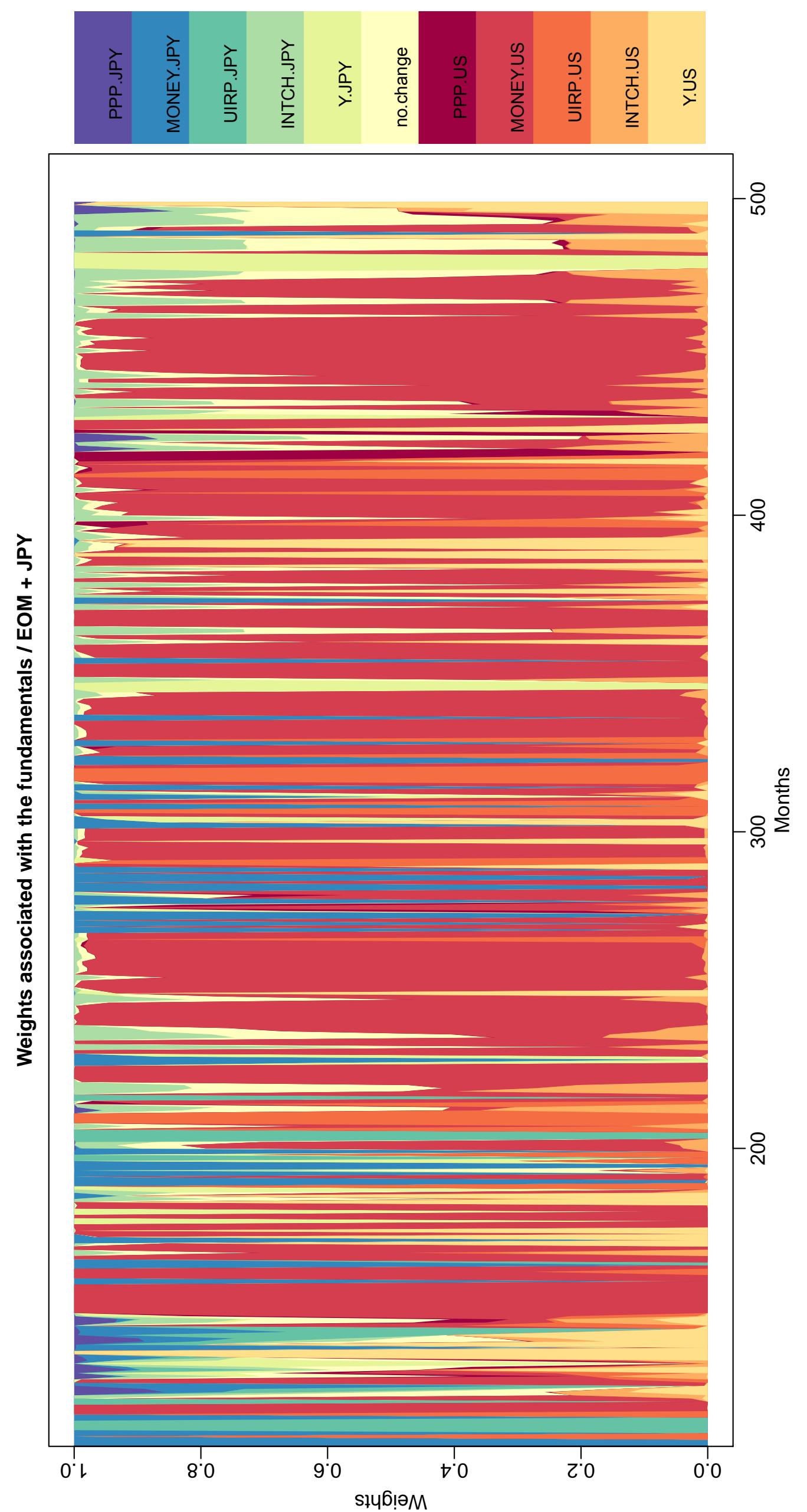

Figure D.2: Weights of different fundamentals in prediction: the JPY/USD case, all fundamentals considered together. 


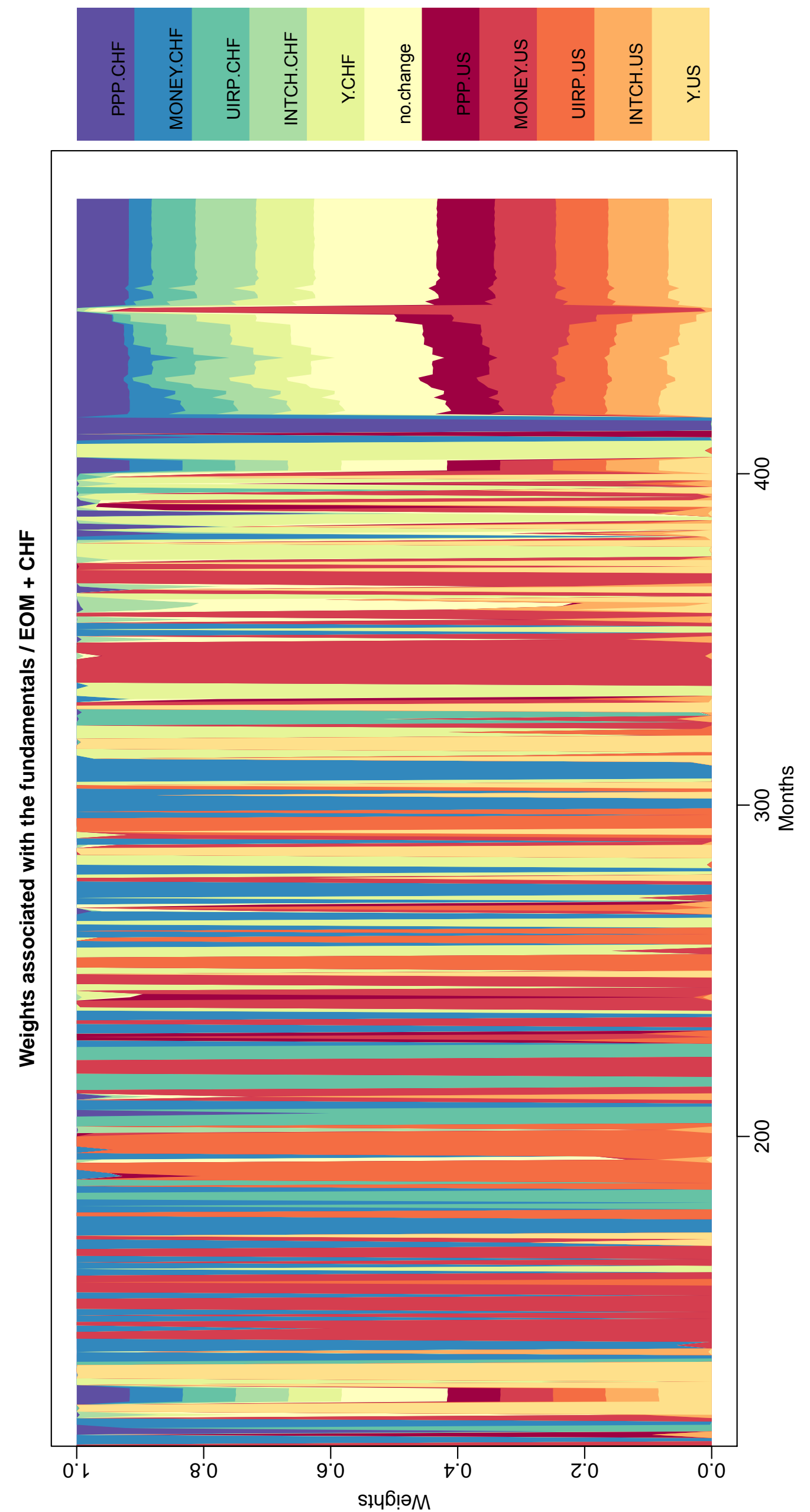

Figure D.3: Weights of different fundamentals in prediction: the CHF/USD case, all fundamentals considered together. 